\title{
Cuspidal part of an Eisenstein series restricted to an index 2 subfield
}

\author{
Yueke $\mathrm{Hu}^{*} \mathbb{0}$
}

${ }^{*}$ Correspondence:

huyueke2012@gmail.com

ETH Zürich, HG GO 68.2,

Rämistrasse 101, 8092 Zurich,

Switzerland

\begin{abstract}
Let $\mathbb{E}$ be a quadratic algebra over a number field $\mathbb{F}$. Let $E(g, s)$ be an Eisenstein series on $G L_{2}(\mathbb{E})$, and let $F$ be a cuspidal automorphic form on $G L_{2}(\mathbb{F})$. We will consider in this paper the following automorphic integral:

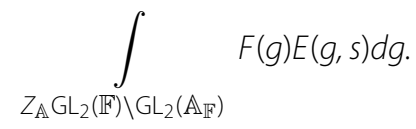

This is in some sense the complementary case to the well-known Rankin-Selberg integral and the triple product formula. We will approach this integral by Waldspurger's formula, giving a criterion about when the integral is automatically zero, and otherwise the L-functions it represents. We will also calculate the local integrals at some ramified places, where the level of the ramification can be arbitrarily large.
\end{abstract}

\section{Background}

In this paper we are interested in the cuspidal part of an Eisenstein series restricted to an index 2 subfield. More specifically, let $\mathbb{E}$ be a quadratic algebra over a number field $\mathbb{F}$. Let $F$ be a cusp form of a cuspidal automorphic representation $\pi$ on $\mathrm{GL}_{2}\left(\mathbb{A}_{\mathbb{F}}\right)$. Let $E(g, s)$ be an Eisenstein series over $\mathbb{E}$, defined from two characters $\chi_{1}$ and $\chi_{2}$ over $\mathbb{E}^{*}$. (see (2.4) for more details of the definition) It is well-known that such Eisenstein series is in the continuous spectrum for $L^{2}\left(\mathrm{GL}_{2}(\mathbb{E}) \backslash \mathrm{GL}_{2}\left(\mathbb{A}_{\mathbb{E}}\right)\right)$. Its integral against a cusp form on $\mathrm{GL}_{2}\left(\mathbb{A}_{\mathbb{E}}\right)$ will simply be zero.

But we are interested in the spectral decomposition of $E(g, s)$ when we restrict it to $\mathrm{GL}_{2}\left(\mathbb{A}_{\mathbb{F}}\right)$. In particular we consider the following integral:

$$
\mathbb{I}(E, F, s)=\int_{Z_{\mathbb{A}} \mathrm{GL}_{2}(\mathbb{F}) \backslash \mathrm{GL}_{2}\left(\mathbb{A}_{\mathbb{F}}\right)} F(g) E(g, s) d g .
$$

This integral is not necessarily zero. We would like to see when this integral is automatically zero and otherwise how $\mathbb{I}(E, F, s)$ depends on $s$.

In addition to its own interest, this automorphic integral is in some sense the complementary case to the well-known Rankin-Selberg integral and triple product formula. It's also a special case of the automorphic integral related to arithmetic height pairing on certain Shimura varieties according to the main theorem in the work of Bruinier, Kudla

(c) The Author(s) 2016. This article is distributed under the terms of the Creative Commons Attribution 4.0 International License (http://creativecommons.org/licenses/by/4.0/), which permits unrestricted use, distribution, and reproduction in any medium, provided you give appropriate credit to the original author(s) and the source, provide a link to the Creative Commons license, and indicate if changes were made. 
and Yang in [4]. The work in this paper may shed some light on how to understand that integral in general.

Let $w_{\pi}$ denote the central character of $\pi$. To avoid triviality, we will assume throughout this paper that

$$
\left.w_{\pi} \cdot\left(\chi_{1} \chi_{2}\right)\right|_{\mathbb{A}_{\mathbb{F}}^{*}}=1 .
$$

Under this assumption, we will relate $\mathbb{I}(E, F, s)$ to certain $L$-functions and special values of $L$-functions. This is not surprising as we have already seen many examples relating automophic integrals and $L$-functions.

\subsection{Automorphic integrals and $L$-functions}

Integral is an important tool to study $L$-functions, as in the earliest example of the integral representation for the Riemann zeta function. It is used to show, for example, the functional equation and the analytic continuation of the $L$ - functions. Tate in his thesis gave the first adelic version of the story. (see [2] as a reference). Let $\mu$ be a Hecke character on $\mathbb{A}_{\mathbb{F}}^{*}$ and $f \in S\left(\mathbb{A}_{\mathbb{F}}\right)$ be a Schwartz function. Tate showed that the integral

$$
\int_{\mathbb{A}_{\mathbb{F}}^{*}} f(x) \mu(x)|x|^{s} d^{*} x
$$

represents the $L$-function of the Hecke character $L(\mu, s)$. His work provided the basic idea to relate the automorphic integrals with the $L$-functions in general: write the automorphic integral as a product of local integrals, then identify the local integrals with the corresponding local $L$-factors for unramified places. The local integral at ramified places could be different from expectation. It depends on, for example, the choice of the Schwartz functions. Thus the global integral could differ from the $L$-function by factors at the set of ramified places, which is finite.

We introduce here two more examples which are similar to (1.1).

\subsubsection{Rankin-Selberg integral}

Let $F_{i}$ be cusp forms over $\mathbb{F}$, coming from automorphic cupidal representations $\pi_{i}$ for $i=1$, 2. Let $E(g, s)$ be the Eisenstein series over $\mathbb{F}$ (not over $\mathbb{E}$ ) associated to two Hecke characters $\chi_{1}$ and $\chi_{2}$ of $\mathbb{A}_{\mathbb{F}}^{*}$. Then the integral

$$
\int_{Z_{\mathbb{A}} \mathrm{GL}_{2}(\mathbb{F}) \backslash \mathrm{GL}_{2}\left(\mathbb{A}_{\mathbb{F}}\right)} F_{1}(g) F_{2}(g) E(g, s) d g
$$

represents (see for example [2])

$$
L\left(\pi_{1} \times \pi_{2}, \chi_{1}, s\right) .
$$

If we specify $\chi_{1}$ to be the trivial character, then we get the standard Rankin-Selberg $L$-function $L\left(\pi_{1} \times \pi_{2}, s\right)$. The Rankin-Selberg method can be applied to more general reductive groups. For a survey on this subject, see for example [3].

\subsubsection{Triple product formula}

Let $\mathbb{B}$ be a quaternion algebra. Let $\pi_{i}$ for $i=1,2,3$ be three irreducible unitary cuspidal automorphic representations of $\mathbb{B}^{*}$. Let $F_{i} \in \pi_{i}$ be cusp forms for $i=1,2$, 3. Let $\Pi$ denote $\pi_{1} \otimes \pi_{2} \otimes \pi_{3}$ in this subsection. Consider the integral 


$$
\int_{Z_{\mathbb{A}} \mathbb{B}^{*}(\mathbb{F}) \backslash \mathbb{B}^{*}(\mathbb{A})} F_{1}(g) F_{2}(g) F_{3}(g) d g .
$$

This integral gives an element of $\operatorname{Hom}_{\mathbb{B}^{*}(\mathbb{A})}(\Pi, \mathbb{C})$, which is at most one dimensional. Prasad in his thesis [20] gave a criterion in terms of local epsilon factors for the local component of $\operatorname{Hom}_{\mathbb{B}^{*}(\mathbb{A})}(\Pi, \mathbb{C})$ to be nonzero. Jacquet then conjectured that the central value

$$
L\left(\pi_{1} \otimes \pi_{2} \otimes \pi_{3}, 1 / 2\right)
$$

of the triple product L-function does not vanish if and only if there exists a quaternion algebra $\mathbb{B}$ and the corresponding $F_{i}$ 's such that (1.5) does not vanish. This conjecture was first proved by Harris and Kudla in $[10,11]$ using an integral representation of triple product L-function (see [6,18]) and the regularized Siegel-Weil formula (see [17]). Later on, more explicit formulae relating $(1.5)$ and $(1.6)$ were given in $[1,8,25]$ for some special cases. Ichino then generalized the above results in [14], where he considered $\Pi$ as an irreducible unitary cuspidal automorphic representations over an étale cubic algebra $\mathbb{K}$ (this in particular includes the case $\Pi=\pi_{1} \otimes \pi_{2} \otimes \pi_{3}$ when $\mathbb{K}$ is just $\mathbb{F} \oplus \mathbb{F} \oplus \mathbb{F}$ ). He showed that a pairing of integral (1.5)

$$
\int_{Z_{\mathbb{A}} \mathbb{B}^{*}(\mathbb{F}) \backslash \mathbb{B}^{*}(\mathbb{A})} F_{1}(g) F_{2}(g) F_{3}(g) d g \int_{Z_{\mathbb{A}} \mathbb{B}^{*}(\mathbb{F}) \backslash \mathbb{B}^{*}(\mathbb{A})} F_{1}^{\prime}(g) F_{2}^{\prime}(g) F_{3}^{\prime}(g) d g
$$

represents

$$
\frac{L(\Pi, 1 / 2)}{L(\Pi, A d, 1)}
$$

\subsubsection{Comparison}

Now we compare the integrals (1.1), (1.4) and (1.5). For simplicity, let $\mathbb{B}$ be the matrix algebra for (1.5). We first consider the case when $\mathbb{E}=\mathbb{F} \oplus \mathbb{F}$ for (1.1), so the Eisenstein series there is a product of two Eisenstein series over $\mathbb{F}$. Then (1.1), (1.4) and (1.5) give a complete list of integrals of possible products of three automorphic forms, either cusp form or Eisenstein series, over $Z_{\mathbb{A}} G_{2}(\mathbb{F}) \backslash G L_{2}\left(\mathbb{A}_{\mathbb{F}}\right)$.

In general for the Rankin-Selberg integral, we can start with a cusp form defined over a quadratic algebra $\mathbb{E}$, restrict it to the base field and integrate it against an Eisenstein series over $\mathbb{F}$. When $\mathbb{E}$ is a quadratic field extension, the integral represents Asai L-function([16]). Similarly for the triple product formula, we can start with a cusp form defined over an étale cubic algebra $\mathbb{K}$, and integrate it over the diagonal $Z_{\mathbb{A}} G L_{2}(\mathbb{F}) \backslash G L_{2}\left(\mathbb{A}_{\mathbb{F}}\right)$. So we have the following table:

\begin{tabular}{lll}
\hline $\begin{array}{l}\text { Degree of the algebra that } \\
\text { the cusp form is defined over }\end{array}$ & $\begin{array}{l}\text { Degree of the algebra that } \\
\text { the Eisenstein series is } \\
\text { defined over }\end{array}$ & L-functions represented \\
\hline 3 & $\begin{array}{l}\text { No Eisenstein series } \\
1\end{array}$ & $\begin{array}{l}\text { Triple product L-function } \\
\text { Rankin-Selberg L-function } \\
\text { or Asai L-function } \\
\text { To be solved in this paper }\end{array}$ \\
\hline
\end{tabular}


Note that we need at least one cusp form to guarantee convergence. So our work on (1.1) is a complementary case to the Rankin-Selberg integral and the triple product formula.

Despite their similarity, we won't follow, for example, Ichino's method directly, as cusp forms and Eisenstein series are somewhat different in nature. It turns out that our integral is more closely related to Waldspurger's period integral (see Sect. 2.4 for its definition and properties).

\subsection{Main results and organization}

If we write $\mathbb{E}=\mathbb{F}(\sqrt{D})$, then we can embed $\mathbb{E}$ into the matrix algebra by

$$
t=a+b \sqrt{D} \mapsto\left(\begin{array}{cc}
a & b \\
b D & a
\end{array}\right)
$$

Let $\eta$ be a quadratic character associated to the quadratic extension $\mathbb{E} / \mathbb{F}$. Recall that the Eisenstein series $E(g, s)$ is associated to two characters $\chi_{1}, \chi_{2}$. For $t \in \mathbb{E}^{*}$, define the character $\Omega$ such that

$$
\Omega(t)=\chi_{1}(\bar{t}) \chi_{2}(t) \text {. }
$$

Define

$$
\chi=\frac{\chi_{1}}{\chi_{2}}
$$

Let $\Pi$ be the base change of $\pi$ to $\mathbb{E}$ in this subsection.

The first goal of this paper is to prove the following theorem:

Theorem 1.1 (1) If $\operatorname{Hom}_{\mathbb{A}_{\mathbb{E}}^{*}}(\pi \otimes \Omega, \mathbb{C})=0$ or $L(\Pi \otimes \Omega, 1 / 2)=0$, then $\mathbb{I}(E, F, s)=0$.

(2) Otherwise, we can fix $F_{1} \in \hat{\pi}$ such that

$$
C=\int_{Z_{\mathbb{A}} \mathbb{E}^{*} \backslash \mathbb{A}_{\mathbb{E}}^{*}} F_{1}\left(t_{1}\right) \Omega^{-1}\left(t_{1}\right) d t_{1} \neq 0
$$

It is independent of $s$ and

$$
\frac{C \cdot \mathbb{I}(E, F, s)}{\left(F_{1}, F\right)}=\frac{\zeta(2) L(\Pi \otimes \Omega, 1 / 2) L\left(\left.\pi \otimes \chi_{1}\right|_{\mathbb{F}^{*},} 2 s+1 / 2\right)}{2 L(\pi, A d, 1) L(\chi, 2 s+1)} \prod_{\nu} \mathbb{P}_{v^{*}}^{0}
$$

Here local integral $\mathbb{P}_{v}^{0}$ is given as in (4.20).

The second goal of this paper is to work out the local integrals $\mathbb{P}_{v}^{0}$ at some ramified places when the global integral is not trivially zero.

Remark 1.2 Using Waldspurger's result, one can at least determine $|C|$ once we specify the choices of $F_{1}, F$. It may seem that one still cannot get an explicit formula even if we compute $\mathbb{P}_{v}^{0}$ explicitly. However one can divide this formula by Waldspurger's formula (see Theorem 2.22 and Corollary 2.24) and get

$$
\frac{\mathbb{I}(E, F, s)}{\int_{Z_{\mathbb{A}} \mathbb{E}^{*} \backslash \mathbb{A}_{\mathbb{E}}^{*}} F(t) \Omega(t) d t}=\frac{L\left(\left.\pi \otimes \chi_{1}\right|_{\mathbb{F}^{*}}, 2 s+1 / 2\right)}{L(\chi, 2 s+1)} \prod_{v} \frac{\mathbb{P}_{v}^{0}}{P_{v}^{0}} .
$$

Here $P_{v}^{0}$ is as in (2.32). Now explicit results on the local integrals $\mathbb{P}_{v}^{0}$ and $P_{v}^{0}$ will allow us to compare our period integrals $\mathbb{I}(E, F, s)$ with Waldspurger's period integral

$$
\int_{Z_{\mathbb{A}} \mathbb{E}^{*} \backslash \mathbb{A}_{\mathbb{E}}^{*}} F(t) \Omega(t) d t
$$


explicitly. This will be used in our future work on span of restriction of Hecke Eisenstein series with levels. (1.11) also allows us to see the main L-functions more clearly.

We will mostly discuss the disjoint ramifications, where the test vectors are chosen to be Gross-Prasad test vectors. But we will also consider a case (Case 5 in Sect. 5) where there are joint ramifications. As a result, locally $\operatorname{Hom}_{\mathbb{E}^{*}}(\pi \otimes \Omega, \mathbb{C}) \neq 0$ in this case and the local new form is a proper test vector. We shall also compute the archimedean place for a special situation in Sect. 6 . As a very special result from these local calculations, we have

Corollary 1.3 Let $\mathbb{E}=\mathbb{Q}(\sqrt{D})$ be a real quadratic extension of $\mathbb{Q}$ for a square-free integer D. Let $N=\prod_{p} p^{c_{p}}>0$ be an integer such that for any $p \mid N$, $p$ is inert in $\mathbb{E}$. Let $F \in \pi$ $F_{1} \in \hat{\pi}$ be anti-holomorphic cuspidal new forms of weight $-2 k$. Let $E$ be a holomorphic new Eisenstein series of parallel weight $(k, k)$, defined by two characters $\chi_{1} \chi_{2}$. Suppose that $\pi$, its central character $w_{\pi}, \chi_{1}, \chi_{1} \mid \mathbb{Q}$ all have finite conductor $N$ and $\chi_{2}$ has finite conductor 1. Then

$$
\frac{C \cdot \mathbb{I}(E, F, s)}{\left(F_{1}, F\right)}=\frac{\zeta(2) L(\Pi \otimes \Omega, 1 / 2) L\left(\left.\pi \otimes \chi_{1}\right|_{\mathbb{F}^{*}}, 2 s+1 / 2\right)}{2 L(\pi, A d, 1) L(\chi, 2 s+1)} \mathbb{P}_{\infty} \prod_{p \mid N} \mathbb{P}_{p^{\prime}}^{0}
$$

where

$$
\begin{aligned}
\mathbb{P}_{\infty} & =\frac{4 \pi}{2 k-1} \frac{1}{(1+D)^{k}} . \\
\mathbb{P}_{p}^{0} & =\frac{1}{(p+1)\left(p^{c_{p}-1}\right) \chi_{1}(\sqrt{D})} .
\end{aligned}
$$

Here $\chi_{1, p}$ is the p-component of the Hecke character $\chi_{1}$.

We refer the readers to Case 5 in Sect. 5 for specific choice of local component of the Eisenstein series in this result. Note that the L-functions here are the standard L-functions (not completed). We have also used the relation between the Tamagawa measure and the measure we shall use for local computations (see (2.7)). The condition about the finite conductor is just to make sure that only Case 5 show up in ramifications. The period integral $C$ also depends on the choice of $D$, which decides the embedding of $\mathbb{E}$ into the matrix algebra.

The tools developed in the local calculation of this paper turns out to be useful for other automorphic integrals. In particular it facilitates the study of the local integral for triple product formula which has direct arithmetic applications to subconvexity bound and equidistribution problems. See [12] and [13] for more details.

The arrangement of this paper is as follows: Sect. 2 will cover some basic definitions, facts and well-known theories. In particular we will review the Weil representations (following [24]), Shimizu's lifting (see [21]). We will also discuss some special elements in the Weil representation and their properties. In Sect. 2.4 we will review Gross and Prasad's test vector, and also two formulations of Waldspurger's formula, one in terms of Shimizu's lifting which we shall use most of time, the other one in terms of matrix coefficient.

In Sect. 3, we will use the standard technique of folding and unfolding to rewrite $\mathbb{I}(E, F, s)$ as

$$
\int_{\mathbb{A}_{\mathbb{E}}^{*} \backslash \mathrm{GL}_{2}(\mathbb{A})} \Phi_{s}\left(\gamma_{0} g\right) \int_{Z_{\mathbb{A}} \mathbb{E}^{*} \backslash \mathbb{A}_{\mathbb{E}}^{*}} F(t g) \Omega(t) d t d g .
$$


This is actually a weighted integral of Waldspurger's period integral. As a corollary,

$$
\left\{\begin{array}{c}
\operatorname{Hom}_{\mathbb{A}_{\mathbb{R}}^{*}}(\pi \otimes \Omega, \mathbb{C})=0 \\
\operatorname{or} L(\Pi \otimes \Omega, 1 / 2)=0
\end{array}\right\} \Longrightarrow \mathbb{I}(E, F, s)=0,
$$

which is part (1) of Theorem 1.1. When this doesn't happen, we can pair (1.13) with a fixed period integral and apply Waldspurger's formula in Theorem 2.22. The simplifying observation is that the weighted integral can be combined with the inner integral of Waldspurger's formula. This leads us to the main identity of Theorem 1.1. It can be formulated in terms of Shimizu lifting or matrix coefficient.

In Sect. 4, we will compute the local integral arising from Sect. 3 for unramified places, i.e. when locally $\pi_{v}$ is unramified, $\mathbb{E}_{v} / \mathbb{F}_{v}$ is either inert or split, and $\Phi_{s}$ is unramified (which in turn implies that $\chi_{1, v}$ and $\chi_{2, v}$ are unramified). We will see in Proposition 4.5 and Proposition 4.6 that the local integral gives the expected L-factors and $\mathbb{P}_{v}^{0}=1$ for unramified places. The work in Sects. 3 and 4 completes the proof of Theorem 1.1.

In Sect. 5, we will do local computations for other non-archimedean places. We will specify certain patterns of ramifications, but the levels of the ramification for $\pi_{v}$ and $\Phi_{s, v}$ can be arbitrary. We will also make sure that the local components of $F_{1}$ and $F$ are either Gross and Prasad's test vectors or local new forms, and keep the calculations easier at the possible cost of using somewhat complicated choice of $\Phi_{s}$.

In Sect. 6, we compute the local integral at real places for the special setting as in Corollary 1.3.

In Appendix we will prove Proposition A.1 which gives better description of the Kirillov model of a supercuspidal representation. This proposition is a key ingredient in the local calculation in Sect. 5.3. It is also important in the local calculations of the triple product formula in [12] and [13].

\section{Notations and preliminary results}

\subsection{Definitions and basic facts}

Let $\mathbb{F}$ denote a number field. Let $\pi$ be an automorphic cuspidal representation of $\mathrm{GL}_{2}$ over $\mathbb{F}$ with the central character $w_{\pi}$. Let $\mathbb{B}$ be a quaternion algebra over $\mathbb{F}$, and $\mathbb{E} / \mathbb{F}$ be a quadratic algebra which is embedded in $\mathbb{B}$. Let $\mathbb{A}_{\mathbb{F}}$ and $\mathbb{A}_{\mathbb{E}}$ be the corresponding adelic rings of $\mathbb{F}$ and $\mathbb{E}$. Without loss of generality we can write $\mathbb{E}$ as $\mathbb{F}(\sqrt{D})$ for $D \in \mathbb{F}$ an algebraic integer. (If $\mathbb{E} \simeq \mathbb{F} \oplus \mathbb{F}$, just take $D=1$.)

In this paper we will be mostly interested in the case when $\mathbb{B}$ is the matrix algebra. In that case, we fix the embedding $\mathbb{E} \hookrightarrow \mathbb{B}$ as follows:

$$
t=a+b \sqrt{D} \mapsto\left(\begin{array}{cc}
a & b \\
b D & a
\end{array}\right) .
$$

Note that the quadratic norm is consistent with the determinant of matrices for this embedding.

Let $\chi_{1}$ and $\chi_{2}$ be two Hecke characters on $\mathbb{E}^{*} \backslash \mathbb{A}_{\mathbb{E}}^{*}$ such that

$$
\left.w_{\pi} \cdot\left(\chi_{1} \chi_{2}\right)\right|_{\mathbb{A}_{\mathbb{F}}^{*}}=1 .
$$

Define

$$
\chi=\frac{\chi_{1}}{\chi_{2}}
$$


Let $\Phi_{s}$ be a section of the induced representation $\operatorname{Ind}_{B}^{\mathrm{GL}_{2}}\left(\chi_{1}, \chi_{2}, s\right)$, where $B$ is the Borel subgroup of $\mathrm{GL}_{2}$. So $\Phi_{s}$ satisfies

$$
\begin{aligned}
& \Phi_{s}\left(\left(\begin{array}{cc}
a_{1} & n \\
0 & a_{2}
\end{array}\right) g\right)=\chi_{1}\left(a_{1}\right) \chi_{2}\left(a_{2}\right)\left|\frac{a_{1}}{a_{2}}\right|_{\mathbb{A}_{\mathbb{E}}}^{s+1 / 2} \Phi_{s}(g) \\
& \text { for all }\left(\begin{array}{cc}
a_{1} & n \\
0 & a_{2}
\end{array}\right) \in B\left(\mathbb{A}_{\mathbb{E}}\right) \text { and } g \in \mathrm{GL}_{2}\left(\mathbb{A}_{\mathbb{E}}\right) \text {. } \\
& \text { Let } \\
& E(g, s)=\sum_{\gamma \in B(\mathbb{E}) \backslash \mathrm{GL}_{2}(\mathbb{E})} \Phi_{s}(\gamma g)
\end{aligned}
$$

be the associated Eisenstein series.

Let $\mathbb{F}_{v}$ be the corresponding local field of $\mathbb{F}$ at a place $v$. Let $K_{v}$ denote the standard maximal compact subgroup of $\mathrm{GL}_{2}\left(\mathbb{F}_{v}\right)$, and

$$
K=\prod_{v} K_{v}
$$

We will call an element of a local representation spherical if it is invariant under $K_{v}$. For unramified representations, there is a unique up to constant spherical element.

When $v$ is a finite place, let $\varpi_{\nu}$ denote a uniformizer of $\mathbb{F}_{v}$. Let $O_{F}$ be the ring of integers of the local field $\mathbb{F}_{v}$, and $O_{E}$ be the ring of integers for $\mathbb{E}_{v}$. Let $v(x)$ denote the valuation of $x \in F_{v}^{*}$. Let $q^{-1}=\left|\varpi_{\nu}\right|_{v}$. For an integer $c>0$, define:

$$
K_{1}\left(\varpi_{v}^{c}\right)=\left\{k \in K_{v} \mid \quad k \equiv\left(\begin{array}{cc}
* & * \\
0 & 1
\end{array}\right) \quad \bmod \left(\varpi_{v}^{c}\right)\right\} .
$$

Similarly denote by $K_{0}\left(\varpi_{v}^{c}\right)$ for those congruent to $\left(\begin{array}{ll}* & * \\ 0 & *\end{array}\right) \bmod \left(\varpi_{v}^{c}\right)$ and $K_{1}^{1}\left(\varpi_{v}^{c}\right)$ for those congruent to $\left(\begin{array}{ll}1 & * \\ 0 & 1\end{array}\right) \bmod \left(\varpi_{v}^{c}\right)$.

We shall pick the Haar measure $d g$ on $Z_{\mathbb{A}} \mathrm{GL}_{2}(\mathbb{F}) \backslash \mathrm{GL}_{2}\left(\mathbb{A}_{\mathbb{F}}\right)$ to be the Tamagawa measure. For simplicity we shall pick the Haar measure $d g_{v}$ at non-archimedean places to be such that the volume of $K_{v}$ is 1 , and the Haar measure at real places to be

$$
\frac{1}{2 \pi} \frac{d a d m d \theta}{a^{2}}
$$

where we write

$$
\mathbb{R}^{*} \backslash \mathrm{GL}_{2}(\mathbb{R})=\left\{\left(\begin{array}{cc}
a & m \\
0 & 1
\end{array}\right) \mid a \in \mathbb{R}^{*}, m \in \mathbb{R}\right\} K
$$

for $K=S O(2)$. Then

$$
d g=\left|\Delta_{\mathbb{F}}\right|^{-3 / 2} \zeta_{\mathbb{F}}^{-1}(2) \prod_{v} d g_{v}
$$

Now we describe the integrals on $\mathrm{GL}_{2}\left(\mathbb{F}_{v}\right)$ when $v$ is finite. These results are easy and probably known by experts.

Lemma 2.1 For every positive integer $c$,

$$
G L_{2}\left(\mathbb{F}_{v}\right)=\coprod_{0 \leq i \leq c} B\left(\begin{array}{cc}
1 & 0 \\
\varpi_{v}^{i} & 1
\end{array}\right) K_{1}\left(\varpi_{v}^{c}\right)
$$


Normalize the Haar measure on $\mathrm{GL}_{2}\left(\mathbb{F}_{v}\right)$ such that $K_{v}$ has volume 1 . Then we have the following:

Lemma 2.2 Locally letf be a $K_{1}\left(\varpi_{v}^{c}\right)$-invariant function, on which the center acts trivially.

Then

$$
\int_{F_{v}^{*} \backslash G L_{2}\left(\mathbb{F}_{v}\right)} f(g) d g=\sum_{0 \leq i \leq c} A_{i} \int_{\mathbb{F}_{v}^{*} \backslash B\left(\mathbb{F}_{v}\right)} f\left(b\left(\begin{array}{cc}
1 & 0 \\
\varpi_{v}^{i} & 1
\end{array}\right)\right) d b .
$$

Here $d b$ is the left Haar measure on $\mathbb{F}_{v}^{*} \backslash B\left(\mathbb{F}_{v}\right)$, and

$$
A_{0}=\frac{q}{q+1}, \quad A_{c}=\frac{1}{(q+1) q^{c-1}} \quad \text { and } \quad A_{i}=\frac{q-1}{(q+1) q^{i}} \quad \text { for } 0<i<c .
$$

Proof For $0 \leq j \leq c$, let $f_{j}$ be the characteristic function of $K_{0}\left(\varpi^{j}\right) . f_{0}$ is just the characteristic function of $K$. Clearly they are all right-invariant under $K_{1}\left(\varpi^{c}\right)$. The integral of these functions just give the volume of these compact subgroups. Suppose that the Haar measure on $\mathrm{GL}_{2}$ are so normalized that the volumes of $K$ and $B\left(O_{F}\right)=B \cap K$ are 1 . The volume of $K_{0}\left(\varpi^{j}\right)$ is $\frac{1}{(q+1) q^{j-1}}$ for $j>0$. On the other hand, we can evaluate the integral by the right hand side of (2.8).

$$
f_{j}\left(b\left(\begin{array}{cc}
1 & 0 \\
\varpi^{i} & 1
\end{array}\right)\right)= \begin{cases}1, & \text { if } b \in B\left(O_{F}\right) \text { and } j \leq i \\
0, & \text { otherwise. }\end{cases}
$$

So

$$
\frac{1}{(q+1) q^{j-1}}=\int_{g \in \mathrm{GL}_{2}} f_{j}(g) d g=\sum_{0 \leq i \leq c} A_{i} \int_{b \in B} f_{j}\left(b\left(\begin{array}{cc}
1 & 0 \\
\varpi^{i} & 1
\end{array}\right)\right) d b=\sum_{j \leq i \leq c} A_{i}
$$

for $0<j \leq c$. When $j=0$, we get

$$
1=\sum_{0 \leq i \leq c} A_{i}
$$

Then it's easy to see that the values of the coefficients $A_{i}$ in the lemma are the only choice.

We also record here some easy results about integrals for additive and multiplicative characters.

Lemma 2.3 Let $\psi_{v}$ be an unramified additive character at $v$. Then

$$
\int_{v(m)=j} \psi_{\nu}(m) d m= \begin{cases}0, & \text { if } j<-1 \\ -1, & \text { if } j=-1 \\ q^{-j}\left(1-q^{-1}\right), & \text { if } j \geq 0\end{cases}
$$

Lemma 2.4 Suppose that $\mu$ is a character of level $k>0$ on $\mathbb{F}_{v}^{*}$. Then

$$
\int_{x \in O_{F}^{*}} \mu\left(1+\varpi^{i} x\right) d x= \begin{cases}0, & \text { if } i<k-1 \\ -q^{-1}, & \text { if } i=k-1 \\ 1-q^{-1}, & \text { if } i \geq k\end{cases}
$$




\subsection{The Weil representation}

The Weil representation can be defined for more general reductive group pairs, but we will focus on the following setting as in [24]:

Fix $\psi$ a nontrivial additive character of $\mathbb{F}$. Let $\mathbb{B}$ be a quaternion algebra over $\mathbb{F}$ with $\iota: x \mapsto \bar{x}$ being the main involution. We can define the reduced norm on $\mathbb{B}$ via

$$
Q(x)=x \iota(x)
$$

We will focus on the case when $\mathbb{B}$ is the matrix algebra $M_{2}(\mathbb{F})$ later on. Denote by $G O(\mathbb{B})$ the orthogonal similitude group of $\mathbb{B}$, with the similitude character $v$. An element $\left(g_{1}, g_{2}\right)$ in $\mathbb{B}^{*} \times \mathbb{B}^{*}$ acts on $\mathbb{B}$ via $\left(g_{1}, g_{2}\right) \cdot x=g_{1} x g_{2}^{-1}$. This actually give us a short exact sequence

$$
1 \rightarrow \mathbb{F}^{*} \rightarrow\left(\mathbb{B}^{*} \times \mathbb{B}^{*}\right) \rtimes\{1, \iota\} \rightarrow G O(\mathbb{B}) \rightarrow 1 .
$$

Here $\mathbb{F}^{*}$ is embedded into the group in the middle by $x \mapsto(x, x) \rtimes 1$. $\iota$ acts on $\mathbb{B}^{*} \times \mathbb{B}^{*}$ by $\left(g_{1}, g_{2}\right) \mapsto\left(\iota\left(g_{2}\right)^{-1}, \iota\left(g_{1}\right)^{-1}\right)$. We will simply write $\left(g_{1}, g_{2}\right)$ for $\left(g_{1}, g_{2}\right) \rtimes 1$ when considered as an element of $G O(\mathbb{B})$.

Definition 2.5 The Weil representation for the similitude group pair $\mathrm{GL}_{2} \times G O(\mathbb{B})$ on the space of Schwartz functions $S\left(\mathbb{B} \times \mathbb{F}^{*}\right)$ is defined as follows: for $f(x, u) \in S\left(\mathbb{B} \times \mathbb{F}^{*}\right)$, $\alpha, \delta \in \mathbb{F}^{*}, \beta \in \mathbb{F}, g \in G O(\mathbb{B})$,

(i) $r^{\prime}\left(\left(\begin{array}{ll}1 & \beta \\ 0 & 1\end{array}\right)\right) f(x, u)=\psi_{u}(\beta Q(x)) f(x, u)$,

(ii) $r^{\prime}\left(\left(\begin{array}{cc}0 & 1 \\ -1 & 0\end{array}\right)\right) f(x, u)=\gamma\left[\psi_{w}, q\right] \int_{\mathbb{B}} f(y, u) \psi_{u}(<x, y>) d y$,

(iii) $r^{\prime}\left(\left(\begin{array}{cc}\alpha & 0 \\ 0 & \alpha^{-1}\end{array}\right)\right) f(x, u)=|\alpha|^{2} f(\alpha x, u)$,

(iv) $r^{\prime}\left(\left(\begin{array}{ll}1 & 0 \\ 0 & \delta\end{array}\right)\right) f(x, u)=|\delta|^{-1} f\left(x, \delta^{-1} u\right)$

(v) $r^{\prime \prime}(g) f(x, u)=f\left(g^{-1} \cdot x, u v(g)\right)$.

Here $\gamma\left[\psi_{u}, q\right]$ equal to 1 if $\mathbb{B}$ is the matrix algebra and -1 is $\mathbb{B}$ is a division algebra. $\psi_{u}(x)=\psi(u x) .\langle x, y\rangle=Q(x+y)-Q(x)-Q(y)$ in (ii).

Remark 2.6 For $\left(g_{1}, g_{2}\right) \in G O(\mathbb{B})$, we have $v\left(g_{1}, g_{2}\right)=Q\left(g_{1}\right) Q\left(g_{2}\right)^{-1}$, and

$$
r^{\prime \prime}\left(g_{1}, g_{2}\right) f(x, u)=f\left(g_{1}^{-1} x g_{2}, u Q\left(g_{1}\right) Q\left(g_{2}\right)^{-1}\right) .
$$

Also by combining (iii) and (iv), we can get

$$
r^{\prime}\left(\left(\begin{array}{ll}
\alpha & 0 \\
0 & 1
\end{array}\right)\right) f(x, u)=|\alpha| f\left(\alpha x, \alpha^{-1} u\right) .
$$

We will use these simple facts later.

\subsubsection{Special elements in the Weil representation}

For a finite place $v$, now we specify $\mathbb{B}_{v}=M_{2}\left(\mathbb{F}_{v}\right)$. We will discuss explicitly some special elements in the Weil representation $S\left(M_{2}\left(\mathbb{F}_{v}\right) \times \mathbb{F}_{v}^{*}\right)$ as given above. 
According to (v) of Definition 2.5,

$$
r^{\prime \prime}(1, g) f(x, u)=f\left(x g, \frac{u}{\operatorname{det} g}\right) \text {. }
$$

We will say a Schwartz function is invariant under the right action of (or just rightinvariant), for example, $K_{1}\left(\varpi_{v}^{c}\right)$, if $r^{\prime \prime}(1, g) f(x, u)=f(x, u)$ for all $g \in K_{1}\left(\varpi_{v}^{c}\right)$.

Denote by $\omega$ the matrix $\left(\begin{array}{cc}0 & 1 \\ -1 & 0\end{array}\right)$. Assume that the local additive character $\psi_{\nu}$ is unramified. Let $x=\left(\begin{array}{ll}x_{1} & x_{2} \\ x_{3} & x_{4}\end{array}\right), y=\left(\begin{array}{ll}y_{1} & y_{2} \\ y_{3} & y_{4}\end{array}\right) \in M_{2}\left(\mathbb{F}_{v}\right)$. By definition,

$$
Q(x)=\operatorname{det} x=x_{1} x_{4}-x_{2} x_{3},
$$

and

$$
\langle x, y\rangle=x_{1} y_{4}+x_{4} y_{1}-x_{2} y_{3}-x_{3} y_{2} .
$$

So

$$
r^{\prime}(\omega) f(x, u)=\int \psi_{v}\left(u\left(x_{1} y_{4}+x_{4} y_{1}-x_{2} y_{3}-x_{3} y_{2}\right)\right) f(y, u) d y .
$$

Lemma 2.7 Let $f=\operatorname{char}\left(\left(\begin{array}{cc}O_{F} & O_{F} \\ O_{F} & O_{F}\end{array}\right)\right)(x) \times \operatorname{char}\left(O_{F}^{*}\right)(u) \in S\left(M_{2}\left(\mathbb{F}_{v}\right) \times \mathbb{F}_{v}^{*}\right)$. It is invariant by $K_{v}$ under both the right action and the Weil representation $r^{\prime}$.

Proof One can check directly.

Remark 2.8 For the conciseness of notations, later on we will simply write, for example, $f=\operatorname{char}\left(\left(\begin{array}{cc}O_{F} & O_{F} \\ O_{F} & O_{F}\end{array}\right)\right) \times \operatorname{char}\left(O_{F}^{*}\right)$ for functions in $S\left(M_{2}\left(\mathbb{F}_{v}\right) \times \mathbb{F}_{v}^{*}\right)$.

Lemma 2.9 Let $f=\operatorname{char}\left(\left(\begin{array}{cc}O_{F} & O_{F} \\ \varpi_{v}^{c} O_{F} & O_{F}\end{array}\right)\right) \times \operatorname{char}\left(O_{F}^{*}\right)$, for integer $c>0$.

(i) It is invariant by $K_{1}\left(\varpi_{v}^{c}\right)$ under both the right action and the Weil representation.

(ii) For $n \in \mathbb{F}_{v}^{*}$ with $0 \leq v(n)=j \leq c$,

$$
\begin{aligned}
r^{\prime}\left(\left(\begin{array}{ll}
1 & 0 \\
n & 1
\end{array}\right)\right) f(x, u)= & q^{j-c} \operatorname{char}\left(\left(\begin{array}{cc}
O_{F} & \varpi_{\nu}^{j-c} O_{F} \\
\varpi_{\nu}^{j} O_{F} & O_{F}
\end{array}\right)\right) \psi_{v}\left(-u x_{2} x_{3} n^{-1}\right) \\
& \times \operatorname{char}\left(O_{F}^{*}\right) .
\end{aligned}
$$

This function is still right $K_{1}\left(\varpi_{v}^{c}\right)$-invariant.

Proof We will prove the formula in (ii) directly. The rest are easy to check. Note that $\left(\begin{array}{ll}1 & 0 \\ n & 1\end{array}\right)=-\omega\left(\begin{array}{cc}1 & -n \\ 0 & 1\end{array}\right) \omega$. Then by definition

$$
\begin{aligned}
r^{\prime}(\omega) f(x, u)= & q^{-c} \operatorname{char}\left(\left(\begin{array}{cc}
O_{F} & \varpi_{\nu}^{-c} O_{F} \\
O_{F} & O_{F}
\end{array}\right)\right) \times \operatorname{char}\left(O_{F}^{*}\right), \\
r^{\prime}\left(\left(\begin{array}{cc}
1 & -n \\
0 & 1
\end{array}\right) \omega\right) f(y, u)= & q^{-c} \operatorname{char}\left(\left(\begin{array}{cc}
O_{F} & \varpi_{\nu}^{-c} O_{F} \\
O_{F} & O_{F}
\end{array}\right)\right) \psi_{\nu}(-u n \operatorname{det} y) \\
& \times \operatorname{char}\left(O_{F}^{*}\right) .
\end{aligned}
$$


Note that $\psi_{v}(-u n \operatorname{det} y)=\psi_{v}\left(u n y_{2} y_{3}\right)$ for $y \in\left(\begin{array}{cc}O_{F} & \varpi_{v}^{-c} O_{F} \\ O_{F} & O_{F}\end{array}\right)$. For another action of $\omega$, the integral in $y_{1} y_{4}$ is very easy. Now we focus on the following integral:

$$
q^{-c} \int_{y_{2} \in \varpi_{\nu}^{-c} O_{F}} \int_{y_{3} \in O_{F}} \psi_{v}\left(u y_{3}\left(n y_{2}-x_{2}\right)\right) \psi_{v}\left(-u x_{3} y_{2}\right) d y_{3} d y_{2} .
$$

Let $x_{2}$ be fixed. For the integral in $y_{3}$ to be non-zero, we need $y_{2} \in n^{-1} x_{2}+\bar{w}_{v}^{-j} O_{F}$ as $v(n)=j$. Then the integral becomes

$$
q^{-c} \int_{y_{2} \in \varpi_{\nu}^{-c} O_{F} \cap n^{-1} x_{2}+\varpi_{\nu}^{-j} O_{F}} \psi_{\nu}\left(-u x_{3} y_{2}\right) d y_{2} .
$$

Note that $\varpi_{v}^{-j} O_{F} \subseteq \varpi_{v}^{-c} O_{F}$. The domain of the integral is not empty iff $x_{2} \in \varpi_{v}^{j-c} O_{F}$. In that case, the integral becomes

$$
q^{-c} \int_{y_{2} \in n^{-1} x_{2}+\varpi_{\nu}^{-j} O_{F}} \psi_{v}\left(-u x_{3} y_{2}\right) d y_{2}=q^{j-c} \psi_{\nu}\left(-u x_{2} x_{3} n^{-1}\right) \text { if } x_{3} \in \varpi_{\nu}^{j} O_{F} .
$$

So we get $r^{\prime}\left(\omega\left(\begin{array}{cc}1 & -n \\ 0 & 1\end{array}\right) \omega\right)=q^{j-c} \operatorname{char}\left(\left(\begin{array}{cc}O_{F} & \varpi_{\nu}^{j-c} O_{F} \\ \varpi_{\nu}^{j} O_{F} & O_{F}\end{array}\right)\right) \psi_{v}\left(-u x_{2} x_{3} n^{-1}\right) \times$ $\operatorname{char}\left(O_{F}^{*}\right)$. Then just note that the action of -1 will not change this function.

Now we consider a slightly different type of Schwartz functions.

Lemma 2.10 Let $b_{1}, b_{2} \in O_{F}$ and c be an integer. Definef $=\operatorname{char}\left(\left(\begin{array}{cc}b_{1}+\varpi_{\nu}^{c} O_{F} & O_{F} \\ b_{2}+\varpi_{v}^{c} O_{F} & O_{F}\end{array}\right)\right)$ $\times \operatorname{char}\left(O_{F}^{*}\right)$.

(i) $f$ is $K_{1}^{1}\left(\varpi_{v}^{c}\right)$-invariant under the Weil representation $r^{\prime}$ and the right action.

(ii) For $n \in \mathbb{F}_{v}^{*}$ with $0 \leq v(n)=j \leq c$,

$$
\begin{aligned}
r^{\prime}\left(\left(\begin{array}{ll}
1 & 0 \\
n & 1
\end{array}\right)\right) f= & q^{2(j-c)} \operatorname{char}\left(\left(\begin{array}{ll}
b_{1}+\varpi_{\nu}^{j} O_{F} & \varpi_{v}^{j-c} O_{F} \\
b_{2}+\varpi_{\nu}^{j} O_{F} & \varpi_{v}^{j-c} O_{F}
\end{array}\right)\right) \\
& \times \psi_{v}\left(u n^{-1}\left[\left(x_{1}-b_{1}\right) x_{4}-x_{2}\left(x_{3}-b_{2}\right)\right]\right) \operatorname{char}\left(O_{F}^{*}\right) .
\end{aligned}
$$

This function is still right $K_{1}^{1}\left(\varpi_{v}^{c}\right)$-invariant.

Proof Similar to the proof in the last lemma.

Remark 2.11 If $f=\operatorname{char}\left(\left(\begin{array}{ll}b_{1}+\varpi_{v}^{c} O_{F} & O_{F} \\ b_{2}+\varpi_{v}^{c} O_{F} & O_{F}\end{array}\right)\right) \times \operatorname{char}\left(\beta+\varpi_{\nu}^{c} O_{F}\right)$ with $\beta \in$ $\left(O_{F} / \varpi_{v}^{c} O_{F}\right)^{*}$, one has a similar result.

\subsection{Shimizu's lifting}

Now we review briefly Shimizu's lifting (see [21] for more details). For the dual group pair $\mathrm{GL}_{2} \times G O(\mathbb{B})$, we can use the Theta lifting to give an automorphic representation of $G O(\mathbb{B})$ corresponding to a given automorphic representation $\pi^{\prime}$ of $\mathrm{GL}_{2}$. One can lift this representation further by the exact sequence (2.11) to an automorphic representation $\Theta\left(\pi^{\prime}\right)$ for $\mathbb{B}^{*} \times \mathbb{B}^{*}$. 
In particular, let $f \in S\left(\mathbb{B}(\mathbb{A}) \times \mathbb{A}_{F}^{*}\right)$ be an element of the Weil representation defined above, $g_{1}, g_{2} \in \mathbb{B}^{*}(\mathbb{A}), \varphi \in \pi^{\prime}$ be a cusp form, and $h \in \mathrm{GL}_{2}\left(\mathbb{A}_{\mathbb{F}}\right)$. The theta kernel is

$$
\theta\left(f, h, g_{1}, g_{2}\right)=\sum_{x \in \mathbb{B}(\mathbb{F}), u \in \mathbb{F}^{*}} r^{\prime}(h) r^{\prime \prime}\left(g_{1}, g_{2}\right) f(x, u) \text {. }
$$

The global Theta lifting is

$$
\theta\left(f, \varphi, g_{1}, g_{2}\right)=\int_{\mathrm{GL}_{2}(\mathbb{F}) \backslash \mathrm{GL}_{2}\left(\mathbb{A}_{\mathbb{F}}\right)} \varphi(h) \theta\left(f, h, g_{1}, g_{2}\right) d h .
$$

The integral is absolutely convergent since $\varphi$ is a cusp form. Then $\Theta\left(\pi^{\prime}\right)$ is just the collection of all such $\theta\left(f, \varphi, g_{1}, g_{2}\right)$ for all possible $\varphi \in \pi^{\prime}$ and $f \in S\left(\mathbb{B}(\mathbb{A}) \times \mathbb{A}_{\mathbb{F}}^{*}\right)$.

Theorem 2.12 (Shimizu's lifting) Let $\pi^{\prime}$ be a cuspidal automorphic representation of $G L_{2}$.

(i) If $\pi^{\prime}$ doesn't appear in the image of Jacquet-Langlands correspondence, then $\Theta\left(\pi^{\prime}\right)=$ 0.

(ii) Otherwise, let $\sigma$ be an automorphic representation of $\mathbb{B}^{*}$ such that $J L(\sigma)=\pi^{\prime}$. Then $\Theta\left(\pi^{\prime}\right)=\sigma \otimes \hat{\sigma}$.

Remark 2.13 In particular this theorem applies to the case when $\mathbb{B}$ is the matrix algebra. In this case, $\mathbb{B}^{*} \simeq \mathrm{GL}_{2}$ and $\sigma \simeq \pi^{\prime}$.

\subsection{Period integral, test vectors and Waldspurger's formula}

\subsubsection{Waldspurger's period integral}

Let $F_{1}$ be an element of $\sigma$, which is an automorphic representation of $\mathbb{B}^{*}$ with the central characters $w_{\sigma}$. Let $\Omega$ be a Hecke character over the quadratic algebra $\mathbb{E}$ such that $\left.\Omega\right|_{\mathbb{A}_{\mathbb{F}}^{*}}=$ $w_{\sigma}$. Waldspurger studied in [24] the following period integral

$$
\int_{Z_{\mathbb{A}} \mathbb{E}^{*} \backslash \mathbb{A}_{\mathbb{E}}^{*}} F_{1}(t) \Omega^{-1}(t) d t .
$$

This period integral actually gives an element in $\operatorname{Hom}_{\mathbb{A}_{\mathbb{E}}^{*}}\left(\sigma \otimes \Omega^{-1}, \mathbb{C}\right)$. But it's not necessary that this space is non-zero.

Now we discuss the local obstruction for this integral to be nonzero. We first need some definitions.

The Hasse invariant $\epsilon\left(\mathbb{B}_{v}\right)$ of a local quaternion algebra $\mathbb{B}_{v}$ is defined to be 1 if $\mathbb{B}_{v} \simeq$ $M_{2}\left(\mathbb{F}_{v}\right)$, and -1 if it's a division algebra. Let $\pi^{\prime}$ be the image of $\sigma$ under Jacquet-Langlands correspondence. Then one can define the local root number $\epsilon\left(\frac{1}{2}, \Pi_{\pi^{\prime}, v} \otimes \Omega_{v}^{-1}\right)$ where $\Pi_{\pi^{\prime}, v}$ is the base change of $\pi_{\nu}^{\prime}$ to $\mathbb{E}_{v}$. In general the local root number would depend on the chosen additive character $\psi_{\nu}$. The condition $\left.\Omega\right|_{\mathbb{A}_{\mathrm{F}}^{*}}=w_{\sigma}$ will guarantee that this local root number is independent of $\psi_{v}$ and only takes values \pm 1 . See [23].

The following theorem is due to Tunnell and Saito $([22,23])$.

Theorem 2.14 The space $\operatorname{Hom}_{\mathbb{E}_{v}^{*}}\left(\sigma_{v} \otimes \Omega_{v}^{-1}, \mathbb{C}\right)$ is at most one-dimensional. It is nonzero if and only if

$$
\epsilon\left(\frac{1}{2}, \Pi_{\pi^{\prime}, v} \otimes \Omega_{v}^{-1}\right)=\Omega_{v}^{-1}(-1) \epsilon\left(\mathbb{B}_{v}\right) .
$$


Example 2.15 Suppose that $\Omega_{v}$ is unramified and $\mathbb{B}_{v} \simeq M_{2}\left(\mathbb{F}_{v}\right)$. So $\pi_{v}^{\prime}=J L\left(\sigma_{v}\right) \simeq \sigma_{v}$. Let $n\left(\pi_{v}^{\prime}\right)$ denote the level of $\pi_{v}^{\prime}$. If $\mathbb{E}_{v}$ is split over $\mathbb{F}_{v}$, then $\epsilon\left(\frac{1}{2}, \Pi_{\pi^{\prime}, v} \otimes \Omega_{v}^{-1}\right)$ is always 1 , and $\operatorname{Hom}_{\mathbb{E}_{v}^{*}}\left(\pi_{v}^{\prime} \otimes \Omega_{v}^{-1}, \mathbb{C}\right)$ is non-zero. If $\mathbb{E}_{v}$ is inert over $\mathbb{F}_{v}, \epsilon\left(\frac{1}{2}, \Pi_{\pi^{\prime}, v} \otimes \Omega_{v}^{-1}\right)=1$ if and only if $n\left(\pi_{v}^{\prime}\right)$ is even. As a result, $\operatorname{Hom}_{\mathbb{E}_{v}^{*}}\left(\pi_{v}^{\prime} \otimes \Omega_{v}^{-1}, \mathbb{C}\right)$ is non-zero if and only if $n\left(\pi_{v}^{\prime}\right)$ is even (see [7] Proposition 6.3.).

\subsubsection{Gross and Prasad's test vector}

If $\operatorname{Hom}_{\mathbb{E}_{v}^{*}}\left(\sigma_{v} \otimes \Omega_{v}^{-1}, \mathbb{C}\right)$ is non-zero for a non-archimedean place, let $l$ be a non-zero element of it. Gross and Prasad in [9] gave a choice of test vector $F_{1, v} \in \sigma_{v}$ such that $l\left(F_{1, v}\right) \neq 0$, under the hypothesis that either $\pi_{v}^{\prime}$ or $\Omega_{v}$ is unramified. This hypothesis implies that the central character is always unramified.

We first assume that $\Omega_{v}$ is unramified. On $\mathbb{B}_{v}$ we have a Trace map defined to be

$$
\operatorname{Tr}(\alpha)=\alpha+\iota(\alpha)
$$

where $\iota$ is the main involution on $\mathbb{B}_{v}$. An order $R$ of $\mathbb{B}_{v}$ is defined to be a subring of $\mathbb{B}_{v}$ containing $O_{F}$ which is a free $O_{F}$-module of rank 4 (equivalently, $R \otimes_{O_{F}} \mathbb{F}_{v}=\mathbb{B}_{v}$ ). Its dual is defined to be

$$
R^{\perp}=\left\{\beta \in \mathbb{B}_{v} \mid \operatorname{Tr}(\alpha \beta) \in O_{F} \text { for all } \alpha \in R\right\} .
$$

Recall $q=\left|\varpi_{v}\right|^{-1}$. Define the reduced discriminant $d(R)$ of $R$ to be the integer such that

$$
\sharp\left(R^{\perp} / R\right)=q^{2 d(R)} .
$$

See [7] for more details.

Let $R_{c}$ be an order of reduced discriminant $c=n\left(\pi_{v}^{\prime}\right)$ which contains $O_{E}$ under the embedding $\mathbb{E}_{v} \hookrightarrow \mathbb{B}_{v}$. It is unique up to conjugacy by $\mathbb{E}_{v}^{*}$. Let $R_{c}^{*}$ denote its units.

Proposition 2.16 Assume that $\Omega_{v}$ is unramified and $\mathbb{B}_{v} \simeq M_{2}\left(\mathbb{F}_{v}\right)$. If $n\left(\pi_{v}^{\prime}\right) \geq 2$, further assume that $\mathbb{E}_{v} / \mathbb{F}_{v}$ is unramified.

When $\operatorname{Hom}_{\mathbb{E}_{v}^{*}}\left(\pi_{v}^{\prime} \otimes \Omega_{v}^{-1}, \mathbb{C}\right) \neq 0$, let l be a non-trivial element of it. Let $F_{v} \in \pi_{v}^{\prime}$ be the unique (up to constant) element fixed by $R_{c}^{*}$. Then $l\left(F_{v}\right) \neq 0$.

Remark 2.17 Proposition 2.16 has statements on the other side of the Jacquet-Langlands correspondence when $\epsilon\left(\frac{1}{2}, \Pi_{\pi^{\prime}, v} \otimes \Omega_{v}^{-1}\right)=-\Omega_{v}^{-1}(-1)$. But we won't record them here as we don't need them.

Example 2.18 Suppose that $\mathbb{B}_{v} \simeq M_{2}\left(\mathbb{F}_{v}\right)$. Suppose that $\mathbb{E}_{v} / \mathbb{F}_{v}$ is inert and can be written as $\mathbb{F}_{v}(\sqrt{D})$. Recall that $\mathbb{E}_{v}$ can be embedded into $M_{2}\left(\mathbb{F}_{v}\right)$ via

$$
a+b \sqrt{D} \mapsto\left(\begin{array}{cc}
a & b \\
b D & a
\end{array}\right) .
$$

By Example 2.15, $\pi_{v}^{\prime} \simeq \sigma_{v}$ should be of even level $c=2 k$. Then we can choose

$$
R_{c}=\left\{\left(\begin{array}{cc}
a+\varpi_{v}^{k} O_{F} & b+\varpi_{v}^{k} O_{F} \\
b D+\varpi_{v}^{k} O_{F} & a+\varpi_{v}^{k} O_{F}
\end{array}\right) \mid a+b \sqrt{D} \in O_{E}\right\} .
$$

Example 2.19 When $\mathbb{E}_{v} / \mathbb{F}_{v}$ is split, $\mathbb{B}_{v}$ must be the matrix algebra and $\pi_{v}^{\prime} \simeq \sigma_{\nu}$. Suppose that 2 is a unit for the local field. For a split place, fix an element $\sqrt{D} \in \mathbb{F}_{v}$ such that $\sqrt{D}^{2}=D$. One can easily check that 


$$
\left(\begin{array}{cc}
1 & -\frac{1}{\sqrt{D}} \\
\sqrt{D} & 1
\end{array}\right)^{-1}\left(\begin{array}{cc}
a & b \\
b D & a
\end{array}\right)\left(\begin{array}{cc}
1 & \frac{1}{-\sqrt{D}} \\
\sqrt{D} & 1
\end{array}\right)=\left(\begin{array}{cc}
a+b \sqrt{D} & 0 \\
0 & a-b \sqrt{D}
\end{array}\right)
$$

We can pick

$$
R_{c}=\left(\begin{array}{cc}
1 & -\frac{1}{\sqrt{D}} \\
\sqrt{D} & 1
\end{array}\right)\left(\begin{array}{cc}
O_{F} & O_{F} \\
\varpi_{\nu}^{c} O_{F} & O_{F}
\end{array}\right)\left(\begin{array}{cc}
1 & -\frac{1}{\sqrt{D}} \\
\sqrt{D} & 1
\end{array}\right)^{-1}
$$

The element fixed by $R_{c}^{*}$ is just the image of the new form under the action of $\pi_{\nu}^{\prime}\left(\left(\begin{array}{cc}1 & -\frac{1}{\sqrt{D}} \\ \sqrt{D} & 1\end{array}\right)\right)$

Now we assume that $\pi_{\nu}^{\prime}$ is unramified and $\Omega_{\nu}$ is ramified of level $c$. This already implies that $\mathbb{B}_{v} \simeq M_{2}\left(\mathbb{F}_{v}\right)$ and $\pi_{v}^{\prime}$ is an unramified principal series. Let $O_{c}=O_{F}+\varpi_{v}^{c} O_{E}$. Let $R$ be a maximal order in $M_{2}\left(\mathbb{F}_{v}\right)$ which optimally contains the order $O_{c}$. This just means that $R$ is maximal and $R \cap \mathbb{E}_{v}=O_{c}$. Such maximal order is unique up to conjugacy by $\mathbb{E}_{v}^{*}$. Similarly we have the following result:

Proposition 2.20 Assume that $\pi_{\nu}^{\prime}$ is unramified and $\Omega_{v}$ is ramified of level $c$.

When $\operatorname{Hom}_{\mathbb{E}_{v}^{*}}\left(\pi_{v}^{\prime} \otimes \Omega_{v}^{-1}, \mathbb{C}\right) \neq 0$, let $l$ be a non-trivial element of it. Let $F_{v} \in \pi_{v}^{\prime}$ be the unique (up to constant) element fixed by $R^{*}$. Then $l\left(F_{v}\right) \neq 0$.

Example 2.21 Suppose that $\mathbb{E}_{v} / \mathbb{F}_{v}$ is inert, $v(D)=0$ and $\mathbb{B}_{v} \simeq M_{2}\left(\mathbb{F}_{v}\right)$. Then we can pick

$$
R=\left\{\left(\begin{array}{cc}
O_{F} & \varpi_{\nu}^{c} O_{F} \\
\varpi_{v}^{-c} O_{F} & O_{F}
\end{array}\right)\right\}
$$

\subsubsection{Waldspurger's formula}

Denote by $\Delta$ the modulus function for $\mathrm{GL}_{2}$ such that

$$
\Delta\left(\left(\begin{array}{cc}
a_{1} & m \\
0 & a_{2}
\end{array}\right) k\right)=\left|\frac{a_{1}}{a_{2}}\right|^{1 / 2} .
$$

Theorem 2.22 (Waldspurger's formula) Let $F_{1} \in \sigma, F_{2} \in \hat{\sigma}$. Let $\varphi \in \pi^{\prime}$ such that $\theta\left(f, \varphi, g_{1}, g_{2}\right)=F_{1}\left(g_{1}\right) F_{2}\left(g_{2}\right)$ under the Shimizu lifting. Let $\Omega$ be a Hecke character of $\mathbb{E}^{*}$ such that $\left.\Omega\right|_{\mathbb{A}_{\mathbb{F}}^{*}}=w_{\sigma}$. Then

$$
\begin{aligned}
& \int_{Z_{\mathbb{A}} \mathbb{E}^{*} \backslash \mathbb{A}_{\mathbb{E}}^{*}} F_{1}\left(t_{1} g_{1}\right) \Omega^{-1}\left(t_{1}\right) d t_{1} \int_{Z_{\mathbb{A}} \mathbb{E}^{*} \backslash \mathbb{A}_{\mathbb{E}}^{*}} F_{2}\left(t_{2} g_{2}\right) \Omega\left(t_{2}\right) d t_{2} \\
& =\left.L(\eta, 1) \int_{N_{\mathbb{A}} Z_{\mathbb{A}} \backslash G L_{2}(\mathbb{A})} \int_{\mathbb{A}_{\mathbb{E}}^{*}} W_{\varphi}^{-}(h) \Delta(h)^{w-1 / 2} r^{\prime}(h) r^{\prime \prime}\left(g_{1}, g_{2}\right) f\left(t, Q(t)^{-1}\right) \Omega(t) d t d h\right|_{w=1 / 2} \\
& =L\left(\Pi_{\pi^{\prime}} \otimes \Omega^{-1}, 1 / 2\right) \prod_{v \in S} P_{0}\left(f_{v}, \Omega_{v}, 1 / 2\right)
\end{aligned}
$$

where $W_{\varphi}^{-}$is the Whittaker function corresponding to $\varphi$ with respect to $\psi^{-}(x)=\psi(-x)$. $\eta$ is the quadratic Hecke character associated to $\mathbb{E} / \mathbb{F}$. $S$ is the finite set of ramified places. $P_{0}\left(f_{v}, \Omega_{v}, w\right)$ is defined as 


$$
\begin{aligned}
P_{0}\left(f_{v}, \Omega_{v}, w\right)= & \frac{L_{v}\left(\eta_{v}, w+1 / 2\right)}{L_{v}\left(\Pi_{\pi^{\prime}, v} \otimes \Omega_{v}^{-1}, w / 2+1 / 4\right)} \\
& \times \int_{N Z \backslash G L_{2}\left(\mathbb{F}_{v}\right)} \int_{\mathbb{E}_{v}^{*}} W_{\varphi}^{-}(h) \Delta(h)^{w-1 / 2} r^{\prime}(h) r^{\prime \prime}\left(g_{1}, g_{2}\right) f\left(t, Q(t)^{-1}\right) \Omega(t) d t d h .
\end{aligned}
$$

One result of Waldspurger's formula is the following:

Corollary 2.23 There exists $F \in \sigma$ such that

$$
\int_{Z_{\mathbb{A}} \mathbb{E}^{*} \backslash \mathbb{A}_{\mathbb{E}}^{*}} F(t) \Omega^{-1}(t) d t \neq 0,
$$

if and only if $\operatorname{Hom}_{\mathbb{A}_{\mathbb{E}}^{*}}\left(\sigma \otimes \Omega^{-1}, \mathbb{C}\right) \neq 0$ and $L\left(\Pi_{\pi^{\prime}} \otimes \Omega^{-1}, 1 / 2\right) \neq 0$.

Waldspurger further considered the integral

$$
B\left(f_{v}, s\right)=\int_{N Z \backslash \mathrm{GL}_{2}\left(\mathbb{F}_{v}\right)} \int_{\mathbb{F}_{v}^{*}} W_{\varphi}^{-}(h) \Delta(h)^{s-1} r^{\prime}(h) f\left(x, x^{-2}\right) w_{\sigma}(x) d^{*} x d h .
$$

He found that there exists a bilinear pairing between $\sigma_{v}$ and its dual, such that

$$
B\left(f_{v}, 1\right)=\left\langle F_{1, v}, F_{2, v}\right\rangle .
$$

And at unramified places,

$$
B\left(f_{v}, s\right)=\frac{L(\pi, A d, s)}{\zeta(2 s)} .
$$

The global pairing can be written as

$$
\left(F_{1}, F_{2}\right)=\int_{\mathbb{B}^{*}(\mathbb{F}) Z(\mathbb{A}) \backslash \mathbb{B}^{*}(\mathbb{A})} F_{1}(g) \overline{F_{2}(g)} d g=\int_{\mathbb{B}^{*}(\mathbb{F}) Z(\mathbb{A}) \backslash \mathbb{B}^{*}(\mathbb{A})} \theta(f, h, g, g) w_{\sigma}^{-1}(g) d g .
$$

For the local pairings defined above, we have the following formula

$$
\left(F_{1}, F_{2}\right)=\frac{2 L(\pi, A d, 1)}{\zeta(2)} \prod_{v} B^{0}\left(f_{v}, 1\right),
$$

where $B^{0}\left(f_{v}, 1\right)=\frac{\zeta(2)}{L(\pi, A d, 1)} B\left(f_{v}, 1\right)$.

Now the point is that when the local integral in Theorem 2.22 is absolutely convergent, it can be rewritten as

$$
P\left(f_{v}, \Omega_{v}, w\right)=\int_{\mathbb{F}_{v}^{*} \backslash \mathbb{E}_{v}^{*}}\left\langle F_{1, v}, \hat{\sigma}_{v}(e) F_{2, v}\right\rangle \Omega_{v}(e) d e .
$$

By combining Theorem 2.22 with (2.30), one can get

Corollary 2.24 For notations as in Theorem 2.22, we have

$$
\begin{aligned}
& \frac{\int_{Z_{\mathbb{A}} \mathbb{E}^{*} \backslash \mathbb{A}_{\mathbb{E}}^{*}} F_{1}\left(t_{1} g_{1}\right) \Omega^{-1}\left(t_{1}\right) d t_{1} \int_{Z_{\mathbb{A}} \mathbb{E}^{*} \backslash \mathbb{A}_{\mathbb{E}}^{*}} F_{2}\left(t_{2} g_{2}\right) \Omega\left(t_{2}\right) d t_{2}}{\left(F_{1}, F_{2}\right)} \\
& \quad=\frac{\zeta(2) L\left(\Pi_{\pi^{\prime}} \otimes \Omega^{-1}, 1 / 2\right)}{2 L(\pi, A d, 1)} \prod_{v} P_{v}^{0} .
\end{aligned}
$$

where

$$
P_{v}^{0}=\frac{L(\pi, A d, 1) L_{v}\left(\eta_{v}, 1\right)}{\zeta(2) L_{v}\left(\Pi_{\pi^{\prime}, v} \otimes \Omega_{v}^{-1}, 1 / 2\right)} \frac{\int_{\mathbb{F}_{v}^{*} \backslash \mathbb{E}_{v}^{*}}\left\langle\sigma_{v}\left(g_{1, v}\right) F_{1, v}, \hat{\sigma}_{v}\left(e g_{2, v}\right) F_{2, v}\right\rangle \Omega_{v}(e) d e}{\left\langle F_{1, v}, F_{2, v}\right\rangle} .
$$


Note that the local terms on the right-hand side are now independent of the normalization of local pairings. So instead of the local pairing defined by (2.27), one can use whichever local pairing that facilitates computations. The local integral is essentially an integral of matrix coefficients.

\section{Global analysis}

In this paper we are interested in the following automorphic integral:

$$
\mathbb{I}(E, F, s)=\int_{Z_{\mathbb{A}} \mathrm{GL}_{2}(\mathbb{F}) \backslash \mathrm{GL}_{2}\left(\mathbb{A}_{\mathbb{F}}\right)} F(g) E(g, s) d g,
$$

where $F$ is an automorphic cusp form over $\mathbb{F}$ and $E(g, s)$ is an Eisenstein series defined over a quadratic algebra $\mathbb{E}$ as in (2.4). We write $\mathbb{E}=\mathbb{F}(\sqrt{D})$ for some algebraic integer $D$.

Lemma 3.1

$$
\mathbb{I}(E, F, s)=\int_{Z_{\mathbb{A}} \mathbb{E}^{*} \backslash G L_{2}(\mathbb{A})} \Phi_{s}\left(\left(\begin{array}{cc}
1 & 0 \\
\sqrt{D} & 1
\end{array}\right) g\right) F(g) d g .
$$

Proof We first decide the double coset representatives of $B(\mathbb{E}) \backslash \mathrm{GL}_{2}(\mathbb{E}) / \mathrm{GL}_{2}(\mathbb{F})$. By Bruhat decomposition,

$$
\mathrm{GL}_{2}(\mathbb{E})=B(\mathbb{E}) \cup\left(\bigcup_{n \in \mathbb{E}} B(\mathbb{E}) \omega\left(\begin{array}{cc}
1 & n \\
0 & 1
\end{array}\right)\right)=B(\mathbb{E}) \cup\left(\bigcup_{m \in \mathbb{E}} B(\mathbb{E})\left(\begin{array}{cc}
1 & 0 \\
m & 1
\end{array}\right) \omega\right)
$$

for $\omega=\left(\begin{array}{cc}0 & 1 \\ -1 & 0\end{array}\right)$. Note that $\omega \in \mathrm{GL}_{2}(\mathbb{F})$. The relation

$$
B(\mathbb{E})\left(\begin{array}{cc}
1 & 0 \\
m_{1} & 1
\end{array}\right) \mathrm{GL}_{2}(\mathbb{F})=B(\mathbb{E})\left(\begin{array}{cc}
1 & 0 \\
m_{2} & 1
\end{array}\right) \mathrm{GL}_{2}(\mathbb{F})
$$

is equivalent to

$$
\left(\begin{array}{cc}
1 & 0 \\
m_{1} & 1
\end{array}\right)\left(\begin{array}{ll}
a & b \\
c & d
\end{array}\right)\left(\begin{array}{cc}
1 & 0 \\
-m_{2} & 1
\end{array}\right) \in B(\mathbb{E})
$$

for some $\left(\begin{array}{ll}a & b \\ c & d\end{array}\right) \in \mathrm{GL}_{2}(\mathbb{F})$. By equating the lower left element of the product to 0 , we get the following condition:

$$
m_{2}=\frac{a m_{1}+c}{b m_{1}+d}
$$

From this one can figure out the double coset representatives and the stabilizers of the right $\mathrm{GL}_{2}(\mathbb{F})$ action for each representative:

(i) Case $m=0$, the stabilizer is $\{c=0\}=N(\mathbb{F})$, the unipotent subgroup. The corresponding orbit is negligible.

(ii) Case $m=\sqrt{D}$, the stabilizer is $\{d=a, c=b D\}=\left\{a I+b\left(\begin{array}{ll}0 & 1 \\ D & 0\end{array}\right)\right\}$, which can be further identified with $\mathbb{E}^{*}$. 
As a result, we can rewrite (3.1) as

$$
\begin{aligned}
\mathbb{I}(E, F, s)= & \int_{Z_{\mathbb{A}} \mathrm{GL}_{2}(\mathbb{F}) \backslash \mathrm{GL}_{2}(\mathbb{A})}\left(\sum_{\alpha \in N(\mathbb{F}) \backslash \mathrm{GL}_{2}(\mathbb{F})} \Phi_{s}\left(\left(\begin{array}{ll}
1 & 0 \\
0 & 1
\end{array}\right) \alpha g\right)\right. \\
& \left.+\sum_{\alpha \in \mathbb{E}^{*} \backslash \mathrm{GL}_{2}(\mathbb{F})} \Phi_{S}\left(\left(\begin{array}{cc}
1 & 0 \\
\sqrt{D} & 1
\end{array}\right) \alpha g\right)\right) F(g) d g \\
= & \int_{Z_{\mathbb{A}} N(\mathbb{F}) \backslash \mathrm{GL}_{2}(\mathbb{A})} \Phi_{s}(g) F(g) d g+\int_{Z_{\mathbb{A}} \mathbb{E}^{*} \backslash \mathrm{GL}_{2}(\mathbb{A})} \Phi_{s}\left(\left(\begin{array}{cc}
1 & 0 \\
\sqrt{D} & 1
\end{array}\right) g\right) F(g) d g .
\end{aligned}
$$

One just has to see that the first term is 0 since $F$ is a cusp form. This is why the corresponding orbit is called negligible.

Denote

$$
\gamma_{0}=\left(\begin{array}{cc}
1 & 0 \\
\sqrt{D} & 1
\end{array}\right)
$$

For $t=\left(\begin{array}{cc}a & b \\ b D & a\end{array}\right) \in \mathbb{A}_{\mathbb{E}}^{*}$, one can check that

$$
\gamma_{0} t \gamma_{0}^{-1}=\left(\begin{array}{cc}
a-b \sqrt{D} & b \\
0 & a+b \sqrt{D}
\end{array}\right)
$$

is actually upper triangular. Recall that $\Phi_{s}$ satisfies

$$
\Phi_{s}\left(\left(\begin{array}{ll}
a & b \\
0 & d
\end{array}\right) x\right)=\chi_{1}(a) \chi_{2}(d)\left|\frac{a}{d}\right|_{\mathbb{A}_{\mathbb{E}}}^{s+1 / 2} \Phi_{s}(x) .
$$

From now on we fix our notation for $\Omega$ as follows:

Definition 3.2 Define for $t \in \mathbb{A}_{\mathbb{E}}^{*}$

$$
\Omega(t)=\chi_{1}(\bar{t}) \chi_{2}(t)=\chi_{1}(a-b \sqrt{D}) \chi_{2}(a+b \sqrt{D}) .
$$

Lemma 3.3 With notations as above, we have $\Phi_{s}\left(\gamma_{0} t g\right)=\Phi_{s}\left(\gamma_{0} g\right) \Omega(t)$ for any $g \in G L_{2}(\mathbb{A})$ and $t \in \mathbb{A}_{\mathbb{E}}^{*}$.

Now we can further write (3.2) as

$$
\mathbb{I}(E, F, s)=\int_{\mathbb{A}_{\mathbb{E}}^{*} \backslash \mathrm{GL}_{2}(\mathbb{A})} \Phi_{s}\left(\gamma_{0} g\right) \int_{Z_{\mathbb{A}} \mathbb{E}^{*} \backslash \mathbb{A}_{\mathbb{E}}^{*}} F(t g) \Omega(t) d t d g .
$$

Note that the interior part of the integral is Waldspurger's period integral to which one can apply Theorem 2.22. The whole integral can be thought of as a weighted integral of Waldspurger's period integral.

To fit into Theorem 2.22, take the quaternion algebra $\mathbb{B}$ there to be $M_{2}(\mathbb{F})$. Pick $F_{2}=$ $F \in \hat{\sigma}=\pi$. Then $F_{1} \in \sigma \simeq \hat{\pi}$ and $\varphi \in \pi^{\prime} \simeq \hat{\pi}$. Pick $\Omega$ as the one we defined above, and pick $g_{1} \equiv 1, g_{2}=g$ in (2.25). Then there are two possible situations: 
First, if $\int_{Z_{\mathbb{A}} \mathbb{E}^{*} \backslash \mathbb{A}_{\mathbb{E}}^{*}} F(\operatorname{tg}) \Omega(t) d t=0$ for any $g$, then $\mathbb{I}(E, F, s)=0$. In particular we have the following corollary:

Corollary 3.4 Let $\Pi$ be the base change of $\pi$ to $\mathbb{E}$. If $\operatorname{Hom}_{\mathbb{A}_{\mathbb{E}}^{*}}(\pi \otimes \Omega, \mathbb{C})=0$ or $L(\Pi \otimes \Omega, 1 / 2)=0$, then $\mathbb{I}(E, F, s)=0$.

Secondly, if $\int_{Z_{\mathbb{A}} \mathbb{E}^{*} \backslash \mathbb{A}_{\mathbb{E}}^{*}} F(t g) \Omega(t) d t$ is not identically zero, one can fix $F_{1} \in \hat{\pi}$ such that the period integral $C=\int_{Z_{\mathbb{A}} \mathbb{E}^{*} \backslash \mathbb{A}_{\mathbb{E}}^{*}} F_{1}\left(t_{1}\right) \Omega^{-1}\left(t_{1}\right) d t_{1}$ is not zero.

Note that since $\Omega$ does not depend on $s$, this fixed period integral $C$ is also independent of $s$. Then by Theorem 2.22 we have the following relation:

$$
\begin{aligned}
C \cdot \mathbb{I}(E, F, s)= & \int_{\mathbb{A}_{\mathbb{E}}^{*} \backslash \mathrm{GL}_{2}(\mathbb{A})} \Phi_{s}\left(\gamma_{0} g\right) \int_{Z_{\mathbb{A}} \mathbb{E}^{*} \backslash \mathbb{A}_{\mathbb{E}}^{*}} F_{1}\left(t_{1}\right) \Omega^{-1}\left(t_{1}\right) d t_{1} \int_{Z_{\mathbb{A}} \mathbb{E}^{*} \backslash \mathbb{A}_{\mathbb{E}}^{*}} F\left(t_{2} g\right) \Omega\left(t_{2}\right) d t_{2} d g \\
= & L(\eta, 1) \int_{\mathbb{A}_{\mathbb{E}}^{*} \backslash \mathrm{GL}_{2}(\mathbb{A})} \Phi_{s}\left(\gamma_{0} g\right) \int_{Z_{\mathbb{A}} N_{\mathbb{A}} \backslash \mathrm{GL}_{2}(\mathbb{A})} \int_{\mathbb{A}_{\mathbb{E}}^{*}} W_{\varphi}^{-}(h) \Delta(h)^{w-1 / 2} r^{\prime}(h) r^{\prime \prime}(1, g) \\
& \times\left. f\left(t, Q(t)^{-1}\right) \Omega(t) d t d h d g\right|_{w=1 / 2} .
\end{aligned}
$$

Recall $\Phi_{s}\left(\gamma_{0} t g\right)=\Phi_{s}\left(\gamma_{0} g\right) \Omega(t)$. By the definition of the Weil representation, in particular by formula (2.12), we have $r^{\prime \prime}(1, g) f\left(t, Q(t)^{-1}\right)=f\left(t g\right.$, $\left.\operatorname{det}(t g)^{-1}\right)$. Then we can actually combine the integrals in $t$ and $g$. This is why we were applying Waldspurger's work in a slightly different way. Using Corollary 2.24, we have

\section{Proposition 3.5 Denote}

$$
\mathbb{I}(E, F, s)=\int_{Z_{\mathbb{A}} G L_{2}(\mathbb{F}) \backslash G L_{2}\left(\mathbb{A}_{\mathbb{F}}\right)} F(g) E(g, s) d g,
$$

where $F$ is an automorphic cusp form over $\mathbb{F}$ and $E(g, s)$ is an Eisenstein series defined over a quadratic algebra $\mathbb{E}$ as in (2.4).

(1) If $\operatorname{Hom}_{\mathbb{A}_{\mathbb{E}}^{*}}(\pi \otimes \Omega, \mathbb{C})=0$ or $L(\Pi \otimes \Omega, 1 / 2)=0$, then $\mathbb{I}(E, F, s)=0$.

(2) Otherwise, we can fix $F_{1} \in \hat{\pi}$, such that

$$
C=\int_{Z_{\mathbb{A}} \mathbb{E}^{*} \backslash \mathbb{A}_{\mathbb{E}}^{*}} F_{1}\left(t_{1}\right) \Omega^{-1}\left(t_{1}\right) d t_{1}
$$

is not zero, independent of s. Let $\varphi \in \hat{\pi}$ and $f$ be a Schwarz function such that $\theta\left(f, \varphi, g_{1}, g_{2}\right)=F_{1}\left(g_{1}\right) F\left(g_{2}\right)$ under the Shimizu lifting. Then we have the following euler products of local integrals:

$$
\begin{aligned}
C \cdot \mathbb{I}(E, F, s)= & L(\eta, 1) \prod_{\nu} \int_{Z N \backslash G L_{2}\left(\mathbb{F}_{v}\right)} \int_{G L_{2}\left(\mathbb{F}_{v}\right)} W_{\varphi, v}^{-}(h) \Delta(h)^{w-1 / 2} r^{\prime}(h) \\
& \times\left. f_{v}\left(g, \operatorname{det}(g)^{-1}\right) \Phi_{s, v}\left(\gamma_{0} g\right) d g d h\right|_{w=1 / 2},
\end{aligned}
$$

or equivalently 


$$
\begin{aligned}
\frac{C \cdot \mathbb{I}(E, F, s)}{\left(F_{1}, F\right)}= & \frac{\zeta(2) L(\eta, 1)}{2 L(\pi, A d, 1)} \prod_{v} \frac{L_{v}(\pi, A d, 1)}{\zeta_{v}(2)} \\
& \times \frac{\left.\int_{Z N \backslash G L_{2}\left(\mathbb{F}_{v}\right)} \int_{G L_{2}\left(\mathbb{F}_{v}\right)} W_{\varphi, v}^{-}(h) \Delta(h)^{w-1 / 2} r^{\prime}(h) f_{v}\left(g, \operatorname{det}(g)^{-1}\right) \Phi_{s, v}\left(\gamma_{0} g\right) d g d h\right|_{w=1 / 2}}{\left.\int_{Z N \backslash G L_{2}\left(\mathbb{F}_{v}\right)} \int_{\mathbb{F}_{v}^{*}} W_{\varphi, v}^{-}(h) \Delta(h)^{w-1 / 2} r^{\prime}(h) f_{v}\left(x, x^{-2}\right) w_{\hat{\pi}}(x) d^{*} x d h\right|_{w=1 / 2}} \\
= & \frac{\zeta(2) L(\eta, 1)}{2 L(\pi, A d, 1)} \prod_{v} \frac{L_{v}(\pi, A d, 1)}{\zeta_{v}(2)} \frac{\int_{\mathbb{F}_{v}^{*} \backslash G L_{2}\left(\mathbb{F}_{v}\right)}\left\langle F_{1, v}, \pi_{v}(g) F_{v}\right\rangle \Phi_{s, v}\left(\gamma_{0} g\right) d g}{\left\langle F_{1, v}, F_{v}\right\rangle} .
\end{aligned}
$$

For the following, we shall denote

$$
\mathbb{P}\left(s, w, f, \Phi_{s}\right)=\int_{Z N \backslash \mathrm{GL}_{2}\left(\mathbb{F}_{v}\right)} \int_{\mathrm{GL}_{2}\left(\mathbb{F}_{v}\right)} W_{\varphi, v}^{-}(h) \Delta(h)^{w-1 / 2} r^{\prime}(h) f_{v}\left(g, \operatorname{det}(g)^{-1}\right) \Phi_{s, v}\left(\gamma_{0} g\right) d g d h .
$$

When $w=\frac{1}{2}$, we also have

$$
\mathbb{P}\left(s, \frac{1}{2}, f, \Phi_{s}\right)=\int_{\mathbb{F}_{v}^{*} \backslash \mathrm{GL}_{2}\left(\mathbb{F}_{v}\right)}\left\langle F_{1, v}, \pi_{v}(g) F_{v}\right\rangle \Phi_{s, v}\left(\gamma_{0} g\right) d g .
$$

For most local calculations, in particular for unramified places, we shall use the first expression. But in some cases we shall also use the second expression when calculations can be made easier.

Remark 3.6 In general, $\varphi$ is not necessarily a newform to have $\theta\left(f, \varphi, g_{1}, g_{2}\right)=F_{1}\left(g_{1}\right) F\left(g_{2}\right)$. But $\varphi$ is always a linear combination of translates of a newform, and we can always make a change of variable in the global Theta lifting to incorporate the translates. So we can choose without loss of generality that $\varphi$ is a newform at the cost of $f$ being possibly more complicated. This explains why we didn't take $\varphi$ as a variable for the integral $\mathbb{P}$.

One potential shortage for the second formulation is that $\left(F_{1}, F\right)$ could be zero while $C \mathbb{I}(E, F, s)$ is nonzero. We will avoid this problem by making $\left(F_{1}, F\right) \neq 0$ for all the local calculations in the following sections.

\section{Local calculations at unramified places}

In the rest sections we will mostly focus on the local integrals, so we will suppress the subscript $v$ to simplify the notations. In this section we will compute the local integral at unramified places. In particular we shall follow the first formula of (3.9) to do computation. Recall that the denominator of (3.9) was already computed by Waldspurger and reviewed in (2.28). So we shall mainly focus on computing $\mathbb{P}\left(s, w, f, \Phi_{s}\right)$.

We specify here what we mean by an unramified place: the quadratic extension $\mathbb{E}$ over $\mathbb{F}$ is either inert or split at this place; $\pi$ is unramified and the corresponding Whittaker function $W_{\varphi}^{-}(h)$ is right $K$-invariant and normalized so that $W_{\varphi}^{-}(1)=1 ; \chi_{i}$ is unramified for $i=1,2$; $\Phi_{s}$ is right $K$-invariant and $\Phi_{s}(1)=1 ; f$ is the Schwartz function

$$
f=\operatorname{char}\left(\left(\begin{array}{ll}
O_{F} & O_{F} \\
O_{F} & O_{F}
\end{array}\right)\right) \times \operatorname{char}\left(O_{F}^{*}\right) .
$$

We always fix an unramified additive character $\psi$ for any non-archimedean places.

We will show in Propositions 4.5 and 4.6 that at unramified places, 


$$
\mathbb{P}\left(s, 1 / 2, f, \Phi_{s}\right)=\frac{L(\Pi \otimes \Omega, 1 / 2) L\left(\left.\pi \otimes \chi_{1}\right|_{\mathbb{F}^{*},} 2 s+1 / 2\right)}{L(\eta, 1) L(\chi, 2 s+1)},
$$

where $\Pi$ is the base change of $\pi, \eta$ is the character associated to the quadratic extension, and $\chi$ as in (2.3) is over $\mathbb{E}$ (so is the corresponding L-function).

We introduce here a few more notations before we start. When $\pi$ is unramified at $v$, let $\pi=\pi\left(\mu_{1}, \mu_{2}\right)$. Then $\varphi \in \hat{\pi} \simeq \pi\left(\mu_{1}^{-1}, \mu_{2}^{-1}\right)$ and the central character of $\hat{\pi}$ satisfies $w_{\hat{\pi}}=\left.\chi_{1, s} \chi_{2, s}\right|_{\mathbb{F}}$. For any multiplicative character $\chi$, we simply write $\chi$ for $\chi(\varpi)$ when there is no confusion. We will also write

$$
\chi_{1, s}=\chi_{1}|\cdot|_{\mathbb{E}}^{s+1 / 2}, \chi_{2, s}=\chi_{2}|\cdot|_{\mathbb{E}}^{-s-1 / 2} .
$$

Then by the definition of $\Phi_{s}$,

$$
\Phi_{s}\left(\left(\begin{array}{cc}
a_{1} & m \\
0 & a_{2}
\end{array}\right) g\right)=\chi_{1, s}\left(a_{1}\right) \chi_{2, s}\left(a_{2}\right) \Phi_{s}(g) \text {. }
$$

We shall also assume without loss of generality that if $\mathbb{E}_{v} / \mathbb{F}_{v}$ is unramified, $D$ is a unit in the local field.

Remark 4.1 First of all, $D$ is a unit for almost all places.

Secondly, suppose everything else are unramified but we use $D^{\prime}=D a^{2}$ for some nonunit $a$. Recall one formulation of the local integral is

$$
\mathbb{P}\left(s, \frac{1}{2}, f, \Phi_{s}\right)^{\prime}=\int_{\mathbb{F}^{*} \backslash \mathrm{GL}_{2}(\mathbb{F})}\left\langle F_{1}, \pi(g) F\right\rangle \Phi_{s}\left(\gamma_{0}^{\prime} g\right) d g .
$$

The only influence of a different $D$ is now

$$
\gamma_{0}^{\prime}=\left(\begin{array}{cc}
1 & 0 \\
\sqrt{D^{\prime}} & 1
\end{array}\right)=\left(\begin{array}{cc}
a^{-1} & 0 \\
0 & 1
\end{array}\right)\left(\begin{array}{cc}
1 & 0 \\
\sqrt{D} & 1
\end{array}\right)\left(\begin{array}{ll}
a & 0 \\
0 & 1
\end{array}\right) .
$$

Denote $\left(\begin{array}{ll}a & 0 \\ 0 & 1\end{array}\right)$ by $m(a)$. Then we have

$$
\begin{aligned}
\mathbb{P}\left(s, \frac{1}{2}, f, \Phi_{s}\right)^{\prime} & =\int_{\mathbb{F}^{*} \backslash \mathrm{GL}_{2}(\mathbb{F})}\left\langle F_{1}, \pi(g) F\right\rangle \Phi_{s}\left(\gamma_{0}^{\prime} g\right) d g \\
& =\int_{\mathbb{F}^{*} \backslash \mathrm{GL}_{2}(\mathbb{F})}\left\langle F_{1}, \pi(g) F\right\rangle \Phi_{s}\left(m(a)^{-1} \gamma_{0} m(a) g\right) d g \\
& =\chi_{1, s}\left(a^{-1}\right) \int_{\mathbb{F}^{*} \backslash \mathrm{GL}_{2}(\mathbb{F})}\left\langle\hat{\pi}(m(a)) F_{1}, \pi(g m(a)) F\right\rangle \Phi_{s}\left(\gamma_{0} g m(a)\right) d g .
\end{aligned}
$$

So if we require $\hat{\pi}(m(a)) F_{1}, \pi(m(a)) F, \Phi_{s}(\cdot m(a))$ to be newforms, we will get exactly the same integral as the $D$ being a unit case. This argument also applies to the ramified cases.

Since $W_{\varphi}^{-}$and $f$ are both right $K$-invariant at unramified places,

$$
\begin{aligned}
\mathbb{P}\left(s, w, f, \Phi_{s}\right)= & \int_{\mathbb{F}^{*}} W_{\varphi}^{-}\left(\left(\begin{array}{ll}
\alpha & 0 \\
0 & 1
\end{array}\right)\right)|\alpha|^{w / 2-1 / 4} \\
& \times \int_{\mathrm{GL}_{2}(\mathbb{F})} r^{\prime}\left(\left(\begin{array}{ll}
\alpha & 0 \\
0 & 1
\end{array}\right)\right) f\left(g, \operatorname{det}(g)^{-1}\right) \Phi_{s}\left(\gamma_{0} g\right) d g|\alpha|^{-1} d^{*} \alpha .
\end{aligned}
$$

By the definition of the Weil representation, in particular by equation (2.13), 


$$
\begin{aligned}
& \int_{\mathrm{GL}_{2}(\mathbb{F})} r^{\prime}\left(\left(\begin{array}{ll}
\alpha & 0 \\
0 & 1
\end{array}\right)\right) f\left(g, \operatorname{det}(g)^{-1}\right) \Phi_{s}\left(\gamma_{0} g\right) d g \\
& =|\alpha| \int_{\mathrm{GL}_{2}(\mathbb{F})} f\left(\alpha g, \alpha^{-1} \operatorname{det}(g)^{-1}\right) \Phi_{s}\left(\gamma_{0} g\right) d g .
\end{aligned}
$$

By substituting $\alpha g \rightarrow g$, we get

$$
\begin{aligned}
& |\alpha| \int_{\mathrm{GL}_{2}(\mathbb{F})} f\left(\alpha g, \alpha^{-1} \operatorname{det}(g)^{-1}\right) \Phi_{s}\left(\gamma_{0} g\right) d g \\
& =|\alpha| \Phi_{s}(\alpha)^{-1} \int_{\mathrm{GL}_{2}(\mathbb{F})} f\left(g, \alpha \operatorname{det}(g)^{-1}\right) \Phi_{s}\left(\gamma_{0} g\right) d g .
\end{aligned}
$$

To be precise, $\Phi_{s}(\alpha)$ here should be understood as $\chi_{1} \chi_{2}(\alpha)$ which is actually independent of $s$. Then the local integral becomes

$$
\int_{\mathbb{F}^{*}} W_{\varphi}^{-}\left(\left(\begin{array}{cc}
\alpha & 0 \\
0 & 1
\end{array}\right)\right)|\alpha|^{w / 2-1 / 4} \Phi_{s}(\alpha)^{-1} \int_{\mathrm{GL}_{2}(\mathbb{F})} f\left(g, \alpha \operatorname{det}(g)^{-1}\right) \Phi_{s}\left(\gamma_{0} g\right) d g d^{*} \alpha .
$$

Denote

$$
I\left(\alpha, f, \Phi_{s}\right)=\int_{\mathrm{GL}_{2}(\mathbb{F})} f\left(g, \alpha \operatorname{det}(g)^{-1}\right) \Phi_{s}\left(\gamma_{0} g\right) d g .
$$

At unramified places $f$ and $\Phi_{s}$ are both right $K$ - invariant, so we just have to do the integral over

$$
B(\mathbb{F})=\left\{\left(\begin{array}{cc}
a_{1} & m \\
0 & a_{2}
\end{array}\right)\right\}
$$

for $I\left(\alpha, f, \Phi_{s}\right)$. Denote

$$
n=v\left(a_{1}\right), \quad k=v(m), \quad l=v\left(a_{2}\right),
$$

where $v(x)$ means the valuation of $x$. By the definition of $f,\left(\left(\begin{array}{cc}a_{1} & m \\ 0 & a_{2}\end{array}\right), \frac{\alpha}{a_{1} a_{2}}\right)$ is in the support of $f$ if and only if $n, l, k \geq 0$ and $\frac{\alpha}{a_{1} a_{2}} \in O_{F}^{*}$. The latter implies $l+n=v(\alpha)$. So

$$
I\left(\alpha, f, \Phi_{s}\right)=\int_{0 \leq n \leq \nu} \int_{k \geq 0} \int_{l=v(\alpha)-n} \Phi_{s}\left(\left(\begin{array}{cc}
1 & 0 \\
\sqrt{D} & 1
\end{array}\right)\left(\begin{array}{cc}
a_{1} & m \\
0 & a_{2}
\end{array}\right)\right) d^{*} a_{2}\left|a_{1}\right|^{-1} d m d^{*} a_{1} .
$$

Here we have used that the left Haar measure for the Borel subgroup is

$$
d^{*} a_{2}\left|a_{1}\right|^{-1} d m d^{*} a_{1} \text {. }
$$

Recall $\gamma_{0}=\left(\begin{array}{cc}1 & 0 \\ \sqrt{D} & 1\end{array}\right)$. One can easily check that

$$
\left(\begin{array}{cc}
1 & 0 \\
\sqrt{D} & 1
\end{array}\right)\left(\begin{array}{cc}
a_{1} & m \\
0 & a_{2}
\end{array}\right)=\left(\begin{array}{cc}
a_{1} & m \\
a_{1} \sqrt{D} & a_{2}+m \sqrt{D}
\end{array}\right)
$$

Lemma 4.2 (1) If $v\left(a_{2}+m \sqrt{D}\right) \geq v\left(a_{1} \sqrt{D}\right)$, then $\Phi_{s}\left(\left(\begin{array}{cc}a_{1} & m \\ a_{1} \sqrt{D} & a_{2}+m \sqrt{D}\end{array}\right)\right)=$ $\chi_{1, s}\left(\frac{a_{2}}{\sqrt{D}}\right) \chi_{2, s}\left(a_{1} \sqrt{D}\right)$. 
(2) If $v\left(a_{2}+m \sqrt{D}\right) \leq v\left(a_{1} \sqrt{D}\right)$, then $\Phi_{s}\left(\left(\begin{array}{cc}a_{1} & m \\ a_{1} \sqrt{D} & a_{2}+m \sqrt{D}\end{array}\right)\right)=\chi_{1, s}\left(\frac{a_{1} a_{2}}{a_{2}+m \sqrt{D}}\right) \chi_{2, s}$ $\left(a_{2}+m \sqrt{D}\right)$.

Proof (1) When $v\left(a_{2}+m \sqrt{D}\right) \geq v\left(a_{1} \sqrt{D}\right)$,

$$
\left(\begin{array}{cc}
a_{1} & m \\
a_{1} \sqrt{D} & a_{2}+m \sqrt{D}
\end{array}\right)=\left(\begin{array}{cc}
\frac{a_{2}}{\sqrt{D}} & a_{1} \\
0 & a_{1} \sqrt{D}
\end{array}\right)\left(\begin{array}{cc}
0 & -1 \\
1 & \frac{a_{2}+m \sqrt{D}}{a_{1} \sqrt{D}}
\end{array}\right) .
$$

(2) When $v\left(a_{2}+m \sqrt{D}\right) \leq v\left(a_{1} \sqrt{D}\right)$,

$$
\left(\begin{array}{cc}
a_{1} & m \\
a_{1} \sqrt{D} & a_{2}+m \sqrt{D}
\end{array}\right)=\left(\begin{array}{cc}
\frac{a_{1} a_{2}}{a_{2}+m \sqrt{D}} & m \\
0 & a_{2}+m \sqrt{D}
\end{array}\right)\left(\begin{array}{cc}
1 & 0 \\
\frac{a_{1} \sqrt{D}}{a_{2}+m \sqrt{D}} & 1
\end{array}\right) .
$$

Then the statements follow from the definition of $\Phi_{s}$ and its right $K$-invariance.

Now we have to consider the inert places separately from the split places.

\subsection{Inert places}

In this subsection we assume that $v$ is an inert place. As a result, $v\left(a_{2}+m \sqrt{D}\right)=\min \{l, k\}$. Note that for this place $\sqrt{D}$ is a unit in the local field. Then by the above lemma, we get

Lemma 4.3 1. If $0 \leq n \leq \frac{v(\alpha)}{2}$, then $l=v(\alpha)-n \geq n$.

(1i) If $k \geq n, \Phi_{s}\left(\left(\begin{array}{cc}a_{1} & m \\ a_{1} \sqrt{D} & a_{2}+m \sqrt{D}\end{array}\right)\right)=\chi_{1, s}\left(\frac{a_{2}}{\sqrt{D}}\right) \chi_{2, s}\left(a_{1} \sqrt{D}\right)=\chi_{1, s}^{v(\alpha)-n} \chi_{2, s}^{n}$.

(1ii) If $0 \leq k<n, \Phi_{s}\left(\left(\begin{array}{cc}a_{1} & m \\ a_{1} \sqrt{D} & a_{2}+m \sqrt{D}\end{array}\right)\right)=\chi_{1, s}\left(\frac{a_{1} a_{2}}{a_{2}+m \sqrt{D}}\right) \chi_{2, s}\left(a_{2}+\right.$ $m \sqrt{D})=\chi_{1, s}^{\nu(\alpha)-k} \chi_{2, s}^{k}$

2. If $\frac{v(\alpha)}{2} \leq n \leq v(\alpha)$, then $l=v(\alpha)-n \leq n$.

(2i) If $k \geq l, \Phi_{s}\left(\left(\begin{array}{cc}a_{1} & m \\ a_{1} \sqrt{D} & a_{2}+m \sqrt{D}\end{array}\right)\right)=\chi_{1, s}\left(\frac{a_{1} a_{2}}{a_{2}+m \sqrt{D}}\right) \chi_{2, s}\left(a_{2}+m \sqrt{D}\right)=$ $\chi_{1, s}^{n} \chi_{2, s}^{\nu(\alpha)-n}$.

(2ii) If $k<l$, $\Phi_{s}\left(\left(\begin{array}{cc}a_{1} & m \\ a_{1} \sqrt{D} & a_{2}+m \sqrt{D}\end{array}\right)\right)=\chi_{1, s}\left(\frac{a_{1} a_{2}}{a_{2}+m \sqrt{D}}\right) \chi_{2, s}\left(a_{2}+m \sqrt{D}\right)=$ $\chi_{1, s}^{v(\alpha)-k} \chi_{2, s^{k}}^{k}$

As $\int_{O_{F}^{*}} d^{*} a=1, \int_{O_{F}^{*}} d m=1-q^{-1}$ and $\left|a_{1}\right|^{-1}=q^{n}$, one can rewrite the integral (4.8) as a summation

$$
\begin{aligned}
I\left(\alpha, f, \Phi_{s}\right)= & \sum_{0 \leq n \leq \frac{\nu(\alpha)}{2}}\left[\sum_{0 \leq k<n} \chi_{1, s}^{\nu(\alpha)-k} \chi_{2, s}^{k} q^{n-k}\left(1-q^{-1}\right)\right. \\
& \left.+\sum_{n \leq k<\infty} \chi_{1, s}^{\nu(\alpha)-n} \chi_{2, s}^{n} q^{n-k}\left(1-q^{-1}\right)\right]
\end{aligned}
$$




$$
\begin{aligned}
& +\sum_{\frac{\nu(\alpha)}{2}<n \leq \nu(\alpha)}\left[\sum_{0 \leq k<v(\alpha)-n} \chi_{1, s}^{\nu(\alpha)-k} \chi_{2, s}^{k} q^{n-k}\left(1-q^{-1}\right)\right. \\
& \left.+\sum_{\nu(\alpha)-n \leq k<\infty} \chi_{1, s}^{n} \chi_{2, s}^{\nu(\alpha)-n} q^{n-k}\left(1-q^{-1}\right)\right] .
\end{aligned}
$$

Then it's a tedious process of summation and combining terms. We will skip the process and give the conclusion directly:

Lemma 4.4 Let $v$ be an inert place. When $v(\alpha)<0, I\left(\alpha, f, \Phi_{s}\right)=0$. When $v(\alpha) \geq 0$,

$$
\begin{aligned}
& I\left(\alpha, f, \Phi_{s}\right)=\chi_{1, s}^{\nu(\alpha)} \frac{1-q^{\nu(\alpha)+1}}{1-q} \frac{1-q^{-1}}{1-\frac{\chi_{2, s}}{q \chi_{1, s}}}+\chi_{1, s}^{\nu(\alpha)} \frac{1-\left(\frac{\chi_{2, s}}{\chi_{1, s}}\right)^{b+1}}{1-\frac{\chi_{2, s}}{\chi_{1, s}}} \frac{q^{-1}\left(1-\frac{\chi_{2, s}}{\chi_{1, s}}\right)}{1-\frac{\chi_{2, s}}{q \chi_{1, s}}} \\
& +\left(q^{-1} \chi_{2, s}\right)^{v(\alpha)} \frac{\left(\frac{q^{2} \chi_{1, s}}{\chi_{2, s}}\right)^{b+1}-\left(\frac{q^{2} \chi_{1, s}}{\chi_{2, s}}\right)^{v(\alpha)+1}}{1-\frac{q^{2} \chi_{1, s}}{\chi_{2, s}}} \frac{q^{-1}\left(1-\frac{\chi_{2, s}}{\chi_{1, s}}\right)}{1-\frac{\chi_{2, s}}{q \chi_{1, s}}} \\
& \text { if } v(\alpha)=2 b, 2 b+1 \\
& =-\frac{q(1+q) \frac{\chi_{1, s}}{\chi_{2, s}}}{1-q^{2} \frac{\chi_{1, s}}{\chi_{2, s}}} \chi_{1, s}^{\nu(\alpha)} q^{\nu(\alpha)} \\
& +\frac{1}{1-q^{2} \frac{\chi_{1, s}}{\chi_{2, s}}} \begin{cases}\left(1+q \frac{\chi_{1, s}}{\chi_{2, s}}\right) \chi_{1, s}^{b} \chi_{2, s}^{b}, & \text { if } v(\alpha)=2 b ; \\
(1+q) \chi_{1, s}^{b+1} \chi_{2, s}^{b}, & \text { if } v(\alpha)=2 b+1 .\end{cases}
\end{aligned}
$$

Now we return to the integral (4.5). For a spherical element $\varphi \in \hat{\pi} \simeq \pi\left(\mu_{1}^{-1}, \mu_{2}^{-1}\right)$, we have

$$
W_{\varphi}^{-}\left(\left(\begin{array}{ll}
\alpha & 0 \\
0 & 1
\end{array}\right)\right)= \begin{cases}|\alpha|^{1 / 2} \frac{\mu_{1}^{-1}(\varpi \alpha)-\mu_{2}^{-1}(\varpi \alpha)}{\mu_{1}^{-1}(\varpi)-\mu_{2}^{-1}(\varpi)}, & \text { if } v(\alpha) \geq 0 \\
0, & \text { otherwise }\end{cases}
$$

By the condition (1.2), we have $\mu_{1} \mu_{2} \chi_{1} \chi_{2}=1$. Denote

$$
\delta=q^{-\left(\frac{w}{2}+\frac{1}{4}\right)} .
$$

We again skip tedious calculations and show results directly:

$$
\mathbb{P}\left(s, w, f, \Phi_{s}\right)=\frac{\left(1+\delta^{2}\right)\left(1-q \frac{\chi_{1, s}}{\chi_{2, s}} \delta^{2}\right)+\left(\mu_{1}+\mu_{2}\right) \chi_{1, s} \delta\left(1-q \delta^{2}\right)}{\left(1-q \mu_{1} \chi_{1, s} \delta\right)\left(1-q \mu_{2} \chi_{1, s} \delta\right)\left(1-\mu_{1}^{2} \chi_{1, s} \chi_{2, s} \delta^{2}\right)\left(1-\mu_{2}^{2} \chi_{1, s} \chi_{2, s} \delta^{2}\right)} .
$$

For a character $\chi$ of $\mathbb{F}^{*}$, define $s(\chi)$ to be the real number such that $|\chi(x)|=|x|^{s(\chi)}$. We have following proposition for the inert case:

Proposition 4.5 Let $v$ be a non-archimedean inert place for $\mathbb{E} / \mathbb{F}$. Suppose that $\operatorname{Re}(s) \geq$ $\left(s\left(\chi_{2}\right)-s\left(\chi_{1}\right)\right) / 4$.

(i) There exists $\epsilon>0$ such that, the integral $\mathbb{P}\left(s, w, f, \Phi_{s}\right)$ converges uniformly in any compact subset of $D=\{w \in \mathbb{C} ; \operatorname{Re}(w)>1 / 2-\epsilon\}$. It's holomorphic in $D$.

(ii) For an unramified place we have:

$$
\mathbb{P}\left(s, w, f, \Phi_{s}\right)=\frac{\left(1+\delta^{2}\right)\left(1-q \frac{\chi_{1, s}}{\chi_{2, s}} \delta^{2}\right)+\left(\mu_{1}+\mu_{2}\right) \chi_{1, s} \delta\left(1-q \delta^{2}\right)}{\left(1-q \mu_{1} \chi_{1, s} \delta\right)\left(1-q \mu_{2} \chi_{1, s} \delta\right)\left(1-\mu_{1}^{2} \chi_{1, s} \chi_{2, s} \delta^{2}\right)\left(1-\mu_{2}^{2} \chi_{1, s} \chi_{2, s} \delta^{2}\right)} .
$$


where $\delta=q^{-\left(\frac{w}{2}+\frac{1}{4}\right)}$. If we evaluate at $w=1 / 2$, and write out variable s explicitly, we get

$$
\begin{aligned}
\mathbb{P}\left(s, 1 / 2, f, \Phi_{s}\right)= & \frac{1+q^{-1}}{\left(1-\mu_{1}^{2} \chi_{1} \chi_{2} q^{-1}\right)\left(1-\mu_{2}^{2} \chi_{1} \chi_{2} q^{-1}\right)} \\
& \times \frac{1-\frac{\chi_{1}}{\chi_{2}} q^{-(4 s+2)}}{\left(1-\mu_{1} \chi_{1} q^{-(2 s+1 / 2)}\right)\left(1-\mu_{2} \chi_{1} q^{-(2 s+1 / 2)}\right)} \\
= & \frac{L(\Pi \otimes \Omega, 1 / 2) L\left(\left.\pi \otimes \chi_{1}\right|_{\mathbb{F}^{*},} 2 s+1 / 2\right)}{L(\eta, 1) L(\chi, 2 s+1)} .
\end{aligned}
$$

Recall $\chi=\frac{\chi_{1}}{\chi_{2}} . L(\chi, 2 s+1)$ here is a product of $L$ factors over all places of $\mathbb{E}$ above $v$. In this case there is only one place with the order of the residue field being $q^{2}$.

Proof For part (1), one can easily imitate Waldspurger's proof for his local integral in [24]. Part (2) follows directly from the calculation above.

\subsection{Split places}

Now we consider the case when $v$ splits into two places $v_{1}$ and $v_{2}$ of $\mathbb{E}$. We will use superscript (1) (2) to denote the component at each of these two places. For simplicity we assume that 2 is a unit, or equivalently $2 \nmid v$. $D$ is now a square in the local field $\mathbb{F}$. Fix one of its square roots and denote it by $\sqrt{D}$ and call the other one $-\sqrt{D}$.

We write

$$
\Phi_{s}=\Phi_{s}^{(1)} \cdot \Phi_{s}^{(2)}
$$

where $\Phi_{s}^{(i)}\left(\left(\begin{array}{cc}a_{1} & m \\ 0 & a_{2}\end{array}\right) g\right)=\chi_{1, s}^{(i)}\left(a_{1}\right) \chi_{2, s}^{(i)}\left(a_{2}\right) \Phi_{s}^{(i)}(g)$ and $\Phi_{s}^{(i)}(1)=1$. Here we denote $\chi_{1, s}^{(i)}=\chi_{1}^{(i)}|\cdot|_{\mathbb{E}_{v_{i}}}^{s+1 / 2}, \chi_{2, s}^{(i)}=\chi_{2}^{(i)}|\cdot|_{\mathbb{E}_{v_{i}}}^{-(s+1 / 2)}$ as in the inert case. In this setting we write

$$
\gamma_{0}=\left(\begin{array}{cc}
1 & 0 \\
(\sqrt{D},-\sqrt{D}) & 1
\end{array}\right)
$$

Similarly

$$
\begin{aligned}
\Omega(t) & =\chi_{1}(a-b \sqrt{D}) \chi_{2}(a+b \sqrt{D}) \\
& =\chi_{1}^{(1)}(a-b \sqrt{D}) \chi_{1}^{(2)}(a+b \sqrt{D}) \chi_{2}^{(1)}(a+b \sqrt{D}) \chi_{2}^{(2)}(a-b \sqrt{D}) \\
& =\chi_{1}^{(1)} \chi_{2}^{(2)}(a-b \sqrt{D}) \chi_{2}^{(1)} \chi_{1}^{(2)}(a+b \sqrt{D}) .
\end{aligned}
$$

We start with $I\left(\alpha, f, \Phi_{s}\right)=\int_{\mathrm{GL}_{2}(\mathbb{F})} f\left(g, \alpha \operatorname{det}(g)^{-1}\right) \Phi_{s}\left(\gamma_{0} g\right) d g$. Recall

$$
n=v\left(a_{1}\right), k=v(m), l=v\left(a_{2}\right) .
$$

Note that $f$ is left invariant by $\left(\begin{array}{cc}1 & 0 \\ \sqrt{D} & 1\end{array}\right)$. By substituting $\left(\begin{array}{cc}1 & 0 \\ -\sqrt{D} & 1\end{array}\right) g \mapsto g$ we get

$$
\begin{aligned}
I\left(\alpha, f, \Phi_{s}\right)= & \int_{\mathrm{GL}_{2}(\mathbb{F})} f\left(g, \alpha \operatorname{det}(g)^{-1}\right) \Phi_{s}^{(1)}\left(\left(\begin{array}{cc}
1 & 0 \\
2 \sqrt{D} & 1
\end{array}\right) g\right) \Phi_{s}^{(2)}(g) d g \\
= & \int_{0 \leq n \leq \nu(\alpha)} \int_{k \geq 0} \int_{l=v(\alpha)-n} \Phi_{s}^{(1)}\left(\left(\begin{array}{cc}
a_{1} & m \\
2 a_{1} \sqrt{D} & a_{2}+2 m \sqrt{D}
\end{array}\right)\right) \chi_{1, s}^{(2)}\left(a_{1}\right) \\
& \times \chi_{2, s}^{(2)}\left(a_{2}\right) d^{*} a_{2}\left|a_{1}\right|^{-1} d m d^{*} a_{1} .
\end{aligned}
$$


Then we can apply Lemma 4.2 for $\Phi_{s}^{(1)}\left(\left(\begin{array}{cc}a_{1} & m \\ 2 a_{1} \sqrt{D} & a_{2}+2 m \sqrt{D}\end{array}\right)\right)$, as $2 \sqrt{D}$ is still a unit. One can further expect Lemma 4.3 to hold mostly, with one exception: according to case (2i) of Lemma 4.3, we expect

$$
\Phi_{s}^{(1)}\left(\left(\begin{array}{cc}
a_{1} & m \\
2 a_{1} \sqrt{D} & a_{2}+2 m \sqrt{D}
\end{array}\right)\right)=\left(\chi_{1, s}^{(1)}\right)^{n}\left(\chi_{2, s}^{(1)}\right)^{\nu(\alpha)-n} .
$$

But when $v\left(a_{2}\right)=v(m)$ in this case, $v\left(a_{2}+2 m \sqrt{D}\right)$ could be larger than $v\left(a_{2}\right)$ or $v(m)$, resulting in a different value for $\Phi_{s}^{(1)}$.

We introduce here a correction term $\Delta I$ for $I\left(\alpha, f, \Phi_{s}\right)$ :

$$
I\left(\alpha, f, \Phi_{s}\right)=I^{\prime}+\Delta I .
$$

Here $I^{\prime}$ is the result one would get if we follow Lemma 4.3 completely. As an analogue of the first expression of (4.10), we have (skipping some tedious steps):

$$
\begin{aligned}
I^{\prime}= & \left(\chi_{1, s}^{(1)} \chi_{2, s}^{(2)}\right)^{v(\alpha)} \frac{1-\left(\frac{q \chi_{1, s}^{(2)}}{\chi_{2, s}^{(2)}}\right)^{v(\alpha)+1}}{1-\frac{q \chi_{1, s}^{(2)}}{\chi_{2, s}^{(2)}}} \frac{1-q^{-1}}{1-\frac{\chi_{2, s}^{(1)}}{q \chi_{1, s}^{(1)}}} \\
& +\left(\chi_{1, s}^{(1)} \chi_{2, s}^{(2)}\right)^{v(\alpha)} \frac{1-\left(\frac{\chi_{1, s}^{(2)} \chi_{2, s}^{(1)}}{\chi_{1, s}^{(1)} \chi_{2, s}^{(2)}}\right)^{b+1}}{1-\frac{\chi_{1, s}^{(2)} \chi_{2, s}^{(1)}}{\chi_{1, s}^{(1)} \chi_{2, s}^{(2)}}} \frac{q^{-1}\left(1-\frac{\chi_{2, s}^{(1)}}{\chi_{1, s}^{(1)}}\right)}{1-\frac{\chi_{2, s}^{(1)}}{q \chi_{1, s}^{(1)}}} \\
& +\left(q^{-1} \chi_{2, s}^{(1)} \chi_{2, s}^{(2)}\right)^{v(\alpha)} \frac{\left(\frac{q^{2} \chi_{1, s}^{(1)} \chi_{1, s}^{(2)}}{\chi_{2, s}^{(1)} \chi_{2, s}^{(2)}}\right)^{b+1}-\left(\frac{q^{2} \chi_{1, s}^{(1)} \chi_{1, s}^{(2)}}{\chi_{2, s}^{(1)} \chi_{2, s}^{(2)}}\right)^{v(\alpha)+1}}{q^{-1}\left(1-\frac{\chi_{2, s}^{(1)}}{\chi_{1, s}^{(1)}}\right)} \\
\chi_{2, s}^{(1)} \chi_{2, s}^{(2)} & 1-\frac{\chi_{2, s}^{(1)}}{q \chi_{1, s}^{(1)}}
\end{aligned}
$$

for $v(\alpha)=2 b, 2 b+1$.

We give here a more detailed description of the correction term. Fix $n$ such that $\frac{\nu(\alpha)}{2}<$ $n \leq v(\alpha)$ and fix $m$ such that $k=v(m)=l$. Consider the integration in $d^{*} a_{2}$ for (4.13), that is,

$$
\int_{v\left(a_{2}\right)=v(\alpha)-n} \Phi_{s}^{(1)}\left(\left(\begin{array}{cc}
a_{1} & m \\
2 a_{1} \sqrt{D} & a_{2}+2 m \sqrt{D}
\end{array}\right)\right) \chi_{1, s}^{(2)}\left(a_{1}\right) \chi_{2, s}^{(2)}\left(a_{2}\right) d^{*} a_{2} .
$$

For a subset of measure $\frac{q-2}{q-1}$ in $a_{2}$,

$$
v\left(a_{2}+2 m \sqrt{D}\right)=k \text { and } \Phi_{s}^{(1)}\left(\left(\begin{array}{cc}
a_{1} & m \\
2 a_{1} \sqrt{D} & a_{2}+2 m \sqrt{D}
\end{array}\right)\right)=\left(\chi_{1, s}^{(1)}\right)^{n}\left(\chi_{2, s}^{(1)}\right)^{v(\alpha)-n} ;
$$

For a subset of measure $\frac{1}{q-1} \frac{q-1}{q}=\frac{1}{q}$ in $a_{2}$,

$$
\begin{aligned}
& v\left(a_{2}+2 m \sqrt{D}\right)=k+1 \text { and } \\
& \Phi_{s}^{(1)}\left(\left(\begin{array}{cc}
a_{1} & m \\
2 a_{1} \sqrt{D} & a_{2}+2 m \sqrt{D}
\end{array}\right)\right)=\left(\chi_{1, s}^{(1)}\right)^{n-1}\left(\chi_{2, s}^{(1)}\right)^{v(\alpha)-n+1} ;
\end{aligned}
$$


and etc. But once $v\left(a_{2}+2 m \sqrt{D}\right) \geq n$, the value of $\Phi_{s}^{(1)}$ will just remain to be $\left(\chi_{1, s}^{(1)}\right)^{v(\alpha)-n}\left(\chi_{2, s}^{(1)}\right)^{n}$. Then the integral (4.15) becomes

$$
\begin{aligned}
\left(\chi_{1, s}^{(1)} \chi_{1, s}^{(2)}\right)^{n}\left(\chi_{2, s}^{(1)} \chi_{2, s}^{(2)}\right)^{v(\alpha)-n}\{ & \frac{q-2}{q-1}+\frac{1}{q} \frac{\chi_{2, s}^{(1)}}{\chi_{1, s}^{(1)}}+\frac{1}{q^{2}}\left(\frac{\chi_{2, s}^{(1)}}{\chi_{1, s}^{(1)}}\right)^{2}+\cdots \\
& \left.+\frac{q}{q^{2 n-v(\alpha)}(q-1)}\left(\frac{\chi_{2, s}^{(1)}}{\chi_{1, s}^{(1)}}\right)^{2 n-v(\alpha)}\right\} .
\end{aligned}
$$

Comparing with the supposed value $\left(\chi_{1, s}^{(1)} \chi_{1, s}^{(2)}\right)^{n}\left(\chi_{2, s}^{(1)} \chi_{2, s}^{(2)}\right)^{\nu(\alpha)-n}$, we get the correction

$$
-\left(\chi_{1, s}^{(1)} \chi_{1, s}^{(2)}\right)^{n}\left(\chi_{2, s}^{(1)} \chi_{2, s}^{(2)}\right)^{\nu(\alpha)-n} \frac{1-\frac{\chi_{2, s}^{(1)}}{\chi_{1, s}^{(1)}}}{1-\frac{\chi_{1, s}^{(1)}}{q \chi_{1, s}^{(1)}}} \frac{1}{q-1}\left(1-\left(\frac{\chi_{2, s}^{(1)}}{q \chi_{1, s}^{(1)}}\right)^{2 n-v(\alpha)}\right) .
$$

Integrating this in $m$ and $a_{1}$ would then give

$$
\begin{aligned}
\Delta I= & -\sum_{\frac{\nu(\alpha)}{2}<n \leq v(\alpha)}\left(q^{-1} \chi_{2, s}^{(1)} \chi_{2, s}^{(2)}\right)^{v(\alpha)}\left(\frac{q^{2} \chi_{1, s}^{(1)} \chi_{1, s}^{(2)}}{\chi_{2, s}^{(1)} \chi_{2, s}^{(2)}}\right)^{n} \frac{q^{-1}\left(1-\frac{\chi_{2, s}^{(1)}}{\chi_{1, s}^{(1)}}\right)}{1-\frac{\chi_{2, s}^{(1)}}{q \chi_{1, s}^{(1)}}} \\
& \times\left(1-\left(\frac{\chi_{2, s}^{(1)}}{q \chi_{1, s}^{(1)}}\right)^{2 n-v(\alpha)}\right) .
\end{aligned}
$$

Then one can check that

$$
\begin{aligned}
I\left(\alpha, f, \Phi_{s}\right)= & \left(\chi_{1, s}^{(1)} \chi_{2, s}^{(2)}\right)^{v(\alpha)} \frac{1-\left(\frac{q \chi_{1, s}^{(2)}}{\chi_{2, s}^{(2)}}\right)^{v(\alpha)+1}}{1-\frac{q \chi_{1, s}^{(2)}}{\chi_{2, s}^{(2)}}} \frac{\frac{\chi_{1, s}^{(1)}}{\chi_{2, s}^{(1)}}(1-q)}{1-q \frac{\chi_{1, s}^{(1)}}{\chi_{2, s}^{(1)}}} \\
& +\left(\chi_{1, s}^{(1)} \chi_{2, s}^{(2)}\right)^{v(\alpha)} \frac{1-\left(\frac{\chi_{1, s}^{(2)} \chi_{2, s}^{(1)}}{\chi_{1, s}^{(1)} \chi_{2, s}^{(2)}}\right)^{v(\alpha)+1}}{1-\frac{\chi_{1, s}^{(2)} \chi_{2, s}^{(1)}}{\chi_{1, s}^{(1)} \chi_{2, s}^{(2)}}} \frac{q^{-1}\left(1-\frac{\chi_{2, s}^{(1)}}{\chi_{1, s}^{(1)}}\right)}{1-\frac{\chi_{2, s}^{(1)}}{q \chi_{1, s}^{(1)}}} .
\end{aligned}
$$

The rest story will be the same as in the inert case, so we will skip some steps and show the results directly. By the assumption on the central character, we have $\mu_{1} \mu_{2} \chi_{1, s}^{(1)} \chi_{2, s}^{(1)} \chi_{1, s}^{(2)} \chi_{2, s}^{(2)}=1$. Recall $\delta=q^{-\left(\frac{w}{2}+\frac{1}{4}\right)}$. Then

$$
\begin{aligned}
\mathbb{P}\left(s, w, f, \Phi_{s}\right) & \\
= & \frac{1-\delta^{2}-\left(\mu_{1}+\mu_{2}\right) \chi_{1, s}^{(1)} \chi_{1, s}^{(2)} \delta\left(1-q \delta^{2}\right)+(1-q)\left(\frac{\chi_{1, s}^{(1)}}{\chi_{2, s}^{(1)}}+\frac{\chi_{1, s}^{(2)}}{\chi_{2, s}^{(2)}}\right) \delta^{2}+\frac{q \chi_{1, s}^{(1)} \chi_{1, s}^{(2)}}{\chi_{2, s}^{(1)}(2)} \delta^{2}\left(1-\delta^{2}\right)}{\left(1-\mu_{1} \chi_{2, s}^{(1)} \chi_{1, s}^{(2)} \delta\right)\left(1-\mu_{1} \chi_{1, s}^{(1)} \chi_{2, s}^{(2)} \delta\right)\left(1-\mu_{2} \chi_{2, s}^{(1)} \chi_{1, s}^{(2)} \delta\right)\left(1-\mu_{2} \chi_{1, s}^{(1)} \chi_{2, s}^{(2)} \delta\right)} \\
& \times \frac{1}{\left(1-q \mu_{1} \chi_{1, s}^{(1)} \chi_{1, s}^{(2)} \delta\right)\left(1-q \mu_{2} \chi_{1, s}^{(1)} \chi_{1, s}^{(2)} \delta\right)} .
\end{aligned}
$$

Recall in the beginning of this subsection we have rewritten $\Omega=\chi_{1}^{(1)} \chi_{2}^{(2)}(a-$ $b \sqrt{D}) \chi_{2}^{(1)} \chi_{1}^{(2)}(a+b \sqrt{D})$. Define $s(\Omega)=s\left(\chi_{1, s}^{(1)} \chi_{2, s}^{(2)}\right)-s\left(\chi_{2, s}^{(1)} \chi_{1, s}^{(2)}\right)=s\left(\chi_{1}^{(1)} \chi_{2}^{(2)}\right)-s\left(\chi_{2}^{(1)} \chi_{1}^{(2)}\right)$. This is independent of $s$. 
Then we have the following proposition:

Proposition 4.6 Let $v$ be a non-archimedean split place for $\mathbb{E} / \mathbb{F}$ and $\delta=q^{-\left(\frac{w}{2}+\frac{1}{4}\right)}$. Suppose that $\operatorname{Re}(s)>\left(s\left(\chi_{2}^{(1)} \chi_{2}^{(2)}\right)-s\left(\chi_{1}^{(1)} \chi_{1}^{(2)}\right)\right) / 4$,

(i) There exists an $\epsilon^{\prime}>0$ such that, the integral $\mathbb{P}\left(s, w, f\right.$, $\left.\Phi_{s}\right)$ converges uniformly in any compact subset of $D^{\prime}=\left\{w \in \mathbb{C} ; \operatorname{Re}(w)>1 / 2+|s(\Omega)|-\epsilon^{\prime}\right\}$. It's holomorphic in $D^{\prime}$.

(ii) For an unramified place we have:

$$
\begin{aligned}
\mathbb{P}\left(s, w, f, \Phi_{s}\right) & \\
= & \frac{1-\delta^{2}-\left(\mu_{1}+\mu_{2}\right) \chi_{1, s}^{(1)} \chi_{1, s}^{(2)} \delta\left(1-q \delta^{2}\right)+(1-q)\left(\frac{\chi_{1, s}^{(1)}}{\chi_{2, s}^{(1)}}+\frac{\chi_{1, s}^{(2)}}{\chi_{2, s}^{(2)}}\right) \delta^{2}+\frac{q \chi_{1, s}^{(1)} \chi_{1, s}^{(2)}}{\chi_{2, s}^{(1)} \chi_{2, s}^{(2)}} \delta^{2}\left(1-\delta^{2}\right)}{\left(1-\mu_{1} \chi_{2, s}^{(1)} \chi_{1, s}^{(2)} \delta\right)\left(1-\mu_{1} \chi_{1, s}^{(1)} \chi_{2, s}^{(2)} \delta\right)\left(1-\mu_{2} \chi_{2, s}^{(1)} \chi_{1, s}^{(2)} \delta\right)\left(1-\mu_{2} \chi_{1, s}^{(1)} \chi_{2, s}^{(2)} \delta\right)} \\
& \times \frac{1}{\left(1-q \mu_{1} \chi_{1, s}^{(1)} \chi_{1, s}^{(2)} \delta\right)\left(1-q \mu_{2} \chi_{1, s}^{(1)} \chi_{1, s}^{(2)} \delta\right)} .
\end{aligned}
$$

When $|s(\Omega)|$ is small enough, we can evaluate at $w=1 / 2$ and write out $s$ explicitly:

$$
\begin{aligned}
\mathbb{P} & \left(s, 1 / 2, f, \Phi_{s}\right) \\
= & \frac{\left(1-q^{-1}\right)}{\left(1-\mu_{1} \chi_{2}^{(1)} \chi_{1}^{(2)} q^{-1 / 2}\right)\left(1-\mu_{1} \chi_{1}^{(1)} \chi_{2}^{(2)} q^{-1 / 2}\right)\left(1-\mu_{2} \chi_{2}^{(1)} \chi_{1}^{(2)} q^{-1 / 2}\right)\left(1-\mu_{2} \chi_{1}^{(1)} \chi_{2}^{(2)} q^{-1 / 2}\right)} \\
& \times \frac{\left(1-\frac{\chi_{1}^{(1)}}{\chi_{2}^{(1)}} q^{-(2 s+1)}\right)\left(1-\frac{\chi_{1}^{(2)}}{\chi_{2}^{(2)}} q^{-(2 s+1)}\right)}{\left(1-\mu_{1} \chi_{1}^{(1)} \chi_{1}^{(2)} q^{-(2 s+1 / 2)}\right)\left(1-\mu_{2} \chi_{1}^{(1)} \chi_{1}^{(2)} q^{-(2 s+1 / 2)}\right)} \\
= & \frac{L(\Pi \otimes \Omega, 1 / 2) L\left(\left.\pi \otimes \chi_{1}\right|_{\mathbb{F}^{*}}, 2 s+1 / 2\right)}{L(\eta, 1) L(\chi, 2 s+1)} .
\end{aligned}
$$

Recall $\chi=\frac{\chi_{1}}{\chi_{2}} . L(\chi, 2 s+1)$ here is a product of $L$ factors over all places of $\mathbb{E}$ above $v$. In the split case there are two places over $v$, thus two factors.

Remark 4.7 Again the proof of part (1) will be very similar to Waldspurger's original proof.

Theorem 4.8 For notations as in Proposition 3.5, we have

$$
\frac{C \cdot \mathbb{I}(E, F, s)}{\left(F_{1}, F\right)}=\frac{\zeta(2) L(\Pi \otimes \Omega, 1 / 2) L\left(\left.\pi \otimes \chi_{1}\right|_{\mathbb{F}^{*}}, 2 s+1 / 2\right)}{2 L(\pi, A d, 1) L(\chi, 2 s+1)} \prod_{v} \mathbb{P}_{v}^{0}\left(s, 1 / 2, f_{v}, \Phi_{s, v}\right),
$$

where for non-archimedean places,

$$
\mathbb{P}_{v}^{0}\left(s, 1 / 2, f_{v}, \Phi_{s, v}\right)=\frac{L_{v}(\pi, A d, 1) L_{v}(\eta, 1) L_{v}(\chi, 2 s+1)}{\zeta_{v}(2) L_{v}(\Pi \otimes \Omega, 1 / 2) L_{v}\left(\left.\pi \otimes \chi_{1}\right|_{\mathbb{F}^{*}}, 2 s+1 / 2\right)} \frac{\mathbb{P}_{v}\left(s, 1 / 2, f_{v}, \Phi_{s, v}\right)}{\left\langle F_{1, v}, F_{v}\right\rangle},
$$

for archimedean places,

$$
\mathbb{P}_{v}^{0}\left(s, 1 / 2, f_{v}, \Phi_{s, v}\right)=\frac{\mathbb{P}_{v}\left(s, 1 / 2, f_{v}, \Phi_{s, v}\right)}{\left\langle F_{1, v}, F_{v}\right\rangle},
$$

where $\mathbb{P}_{v}\left(s, 1 / 2, f_{v}, \Phi_{s, v}\right)$ is as in (3.10) or alternatively (3.11).

The product in $v$ is a finite product since $\mathbb{P}_{v}^{0}\left(s, 1 / 2, f_{v}, \Phi_{s, v}\right)=1$ for almost all places due to Propositions 4.5, 4.6 and the local unramified calculations done in [24]. 


\section{Local calculations for other non-archimedean places}

In this section we will compute $\mathbb{P}^{0}\left(s, w, f, \Phi_{s}\right)$ for some ramified non-archimedean places. The additive character $\psi$ is assumed without loss of generality to be unramified, as the formulation of the local integral (3.11) is completely independent of the choice of $\psi$. As explained in Remark 4.1, we assume $v(D)=0$ if $\mathbb{E}_{v} / \mathbb{F}_{v}$ is unramified, and $v(D)=1$ otherwise. Before we start, we first list the ramified cases we are going to consider in this section.

\begin{tabular}{llll}
\hline Case & $\pi$ & $\chi_{1}$ and $\chi_{2}$ & $\mathbb{E} / \mathbb{F}$ \\
\hline 1 & Unramified & Unramified & Ramified \\
2 & Unramified special & Unramified & Split \\
3 & Highly ramified of level $c$ & Unramified & Split \\
4 & Unramified & $\chi_{1}$ level c & Inert \\
5 & $\mu_{2}$ level c & $\chi_{1}$ level c & Inert \\
6 & Highly ramified of even level $c$ & Unramified & Inert \\
\hline
\end{tabular}

The characters not mentioned (that is, $\mu_{1}$ and $\chi_{2}$ ) in Cases 4 and 5 are all unramified. This implies that $\left.\chi_{1}\right|_{\mathbb{F}^{*}}$ is unramified in Case 4 and is of level $c$ in Case 5 . Here by $\pi$ highly ramified, we mean $\pi$ is of level $c \geq 2$.

This section will be organized to solve these cases one by one. It may seem that Case 6 should be done earlier. But we are going to use a different approach, so we leave it to the last.

In Case 1 to Case 5, we will also need to keep track of the denominator of the local integral using (2.27) that

$$
\left\langle F_{1}, F\right\rangle=\int_{N Z \backslash \mathrm{GL}_{2}\left(\mathbb{F}_{v}\right)} \int_{\mathbb{F}_{v}^{*}} W_{\varphi}^{-}(h) r^{\prime}(h) f\left(x, x^{-2}\right) w_{\hat{\pi}}(x) d^{*} x d h .
$$

We are going to get the following table of normalized local integral:

\begin{tabular}{lll}
\hline Case & Choice of $F_{1}, F$ & $\mathbb{P}_{v}^{0}\left(s, 1 / 2, f, \Phi_{s}\right)$ \\
\hline 1 & G-P test vector & 1 \\
2 & G-P test vector & $\frac{1}{q\left(1-\chi^{(2)} q^{-(2 s+1)}\right)}$ \\
3 & G-P test vector & $\frac{1}{q^{c}} \frac{L(\pi, A d, 1)}{1-\chi^{(2)} q^{-(2 s+1)}}$ \\
4 & G-P test vector & $\frac{\mathbb{P}^{\prime}(1 / 2)}{1+q^{-1}}$ for $\mathbb{P}^{\prime}(w)$ given in (5.66) \\
5 & new form & $\frac{1}{(q+1) q^{c-1} \chi_{1}(\sqrt{D})}$ \\
6 & G-P test vector & $\frac{L(\pi, A d, 1)}{q^{c}\left(1-\chi q^{-(4 s+2)}\right)}$ \\
\hline
\end{tabular}

Recall here that $\chi=\frac{\chi_{1}}{\chi_{2}}$.

Remark 5.1 1. When $c$ is odd and the quadratic extension is inert, the local integral is automatically zero according to Example 2.15. Thus Case 2,3,6 will cover all situations when only $\pi$ is ramified. When $\chi_{1}$ and/or $\chi_{2}$ are ramified, the situation could be very complicated. So we restrict ourselves to Case 4 and 5 only. 
2. $\varphi$ is always a newform. G-P test vector above refers to the Gross and Prasad's test vectors as discussed in Subsect. 2.4.2. Note that from Case 1 to Case 5 we are going to use Theta lifting for a Schwartz function $f$ and in this table we claim that we are using G-P test vector/new form. This is simply because the Schwartz function $f$ we will choose has corresponding left/right invariance, and G-P test vector/new form is unique up to constant by such invariance. For the specific choices of $f$ and $\Phi_{s}$, we refer the readers to each subsections. We are going to keep $F_{1} F$ and calculations as simple as possible, at the cost of sometimes complicated choices of $\Phi_{s}$.

3. $L(\pi, A d, 1)$ is not given explicitly in Cases 3 and 6 as it could be different for highly ramified principal series and supercuspidal representations, while we wish to keep the formula uniform.

One corollary for Case 5 above is the following new result after Gross and Prasad's work:

Corollary 5.2 Suppose that the local field extension $\mathbb{E} / \mathbb{F}$ is inert and that $\pi, w_{\pi}, \Omega,\left.\Omega\right|_{\mathbb{F}}$ have same levels. Then

$$
\operatorname{Hom}_{\mathbb{E}^{*}}(\pi \otimes \Omega, \mathbb{C}) \neq 0,
$$

and the local new form for $\pi$ is a test vector for any nonzero element from this space.

\section{$5.1 \mathbb{E} / \mathbb{F}$ ramified}

Here we consider the case when $\pi$ and $\Phi_{s}$ are both unramified, but $\mathbb{E} / \mathbb{F}$ is a ramified local field extension. Let $\varpi_{\mathbb{E}}$ be a uniformizer of $\mathbb{E}$ such that $\varpi_{\mathbb{E}}^{2}=\varpi$. For simplicity, we still let $v(\varpi)=1$ and write $\mu$ or $\chi$ in short for $\mu(\varpi)$ or $\chi(\varpi)$. We suppose that $v(\sqrt{D})=\frac{1}{2}$. We will prove in this subsection the following result

Proposition 5.3 Suppose that $\pi$ and $\Phi_{s}$ are both unramified at $v, \mathbb{E} / \mathbb{F}$ is ramified with $v(\sqrt{D})=1 / 2$. We pick $f$ and $\Phi_{s}$ as in the unramified case. Then

$$
\mathbb{P}\left(s, w, f, \Phi_{s}\right)=\frac{\left[1+\left(\mu_{1}^{2} \chi_{1, s} \chi_{2, s}\right)\left(\varpi_{E}\right) \delta+\left(\mu_{2}^{2} \chi_{1, s} \chi_{2, s}\right)\left(\varpi_{E}\right) \delta+\delta^{2}\right]\left(1-q \delta^{2} \frac{\chi_{1, s}}{\chi_{2, s}}\left(\varpi_{E}\right)\right)}{\left(1-q \mu_{1} \chi_{1, s} \delta\right)\left(1-q \mu_{2} \chi_{1, s} \delta\right)\left(1-\mu_{1}^{2} \chi_{1, s} \chi_{2, s} \delta^{2}\right)\left(1-\mu_{2}^{2} \chi_{1, s} \chi_{2, s} \delta^{2}\right)}
$$

for $\delta=q^{-\left(\frac{w}{2}+\frac{1}{4}\right)}$. When $w=\frac{1}{2}$,

$$
\begin{aligned}
\mathbb{P}\left(s, \frac{1}{2}, f, \Phi_{s}\right)= & \frac{1}{\left(1-\left(\mu_{1}^{2} \chi_{1} \chi_{2}\right)\left(\varpi_{E}\right) q^{-\frac{1}{2}}\right)\left(1-\left(\mu_{2}^{2} \chi_{1} \chi_{2}\right)\left(\varpi_{E}\right) q^{-\frac{1}{2}}\right)} \\
& \times \frac{1-\frac{\chi_{1}}{\chi_{2}}\left(\varpi_{E}\right) q^{-(2 s+1)}}{\left(1-\mu_{1} \chi_{1} q^{-(2 s+1 / 2)}\right)\left(1-\mu_{2} \chi_{1} q^{-(2 s+1 / 2)}\right)}
\end{aligned}
$$

is just as expected. Thus $\mathbb{P}^{0}\left(s, 1 / 2, f, \Phi_{s}\right)=1$.

To compare with the unramified case, we write $\chi_{i, s}^{\frac{1}{2}}$ to mean $\chi_{i, s}\left(\varpi_{E}\right)$. As in the inert case, we can start with Eqs. (4.5) and (4.8). Lemma 4.2 still holds. Then we have the following lemma as an analogue of Lemma 4.3:

Lemma 5.4 (1) If $0 \leq n<\frac{v(\alpha)}{2}$, then $l>n$. 
(1i) If $k \geq n, \Phi_{s}\left(\left(\begin{array}{cc}a_{1} & m \\ a_{1} \sqrt{D} & a_{2}+m \sqrt{D}\end{array}\right)\right)=\chi_{1, s}\left(\frac{a_{2}}{\sqrt{D}}\right) \chi_{2, s}\left(a_{1} \sqrt{D}\right)=\chi_{1, s}^{v(\alpha)-n-\frac{1}{2}}$ $\chi_{2, s}^{n+\frac{1}{2}}$

(1ii) If $0 \leq k<n, \Phi_{s}\left(\left(\begin{array}{cc}a_{1} & m \\ a_{1} \sqrt{D} & a_{2}+m \sqrt{D}\end{array}\right)\right)=\chi_{1, s}\left(\frac{a_{1} a_{2}}{a_{2}+m \sqrt{D}}\right) \chi_{2, s}\left(a_{2}+\right.$ $m \sqrt{D})=\chi_{1, s}^{v(\alpha)-k-\frac{1}{2}} \chi_{2, s}^{k+\frac{1}{2}}$.

(2) If $\frac{v(\alpha)}{2} \leq n \leq v(\alpha)$, then $l \leq n$.

(2i) If $k \geq l, \Phi_{s}\left(\left(\begin{array}{cc}a_{1} & m \\ a_{1} \sqrt{D} & a_{2}+m \sqrt{D}\end{array}\right)\right)=\chi_{1, s}\left(\frac{a_{1} a_{2}}{a_{2}+m \sqrt{D}}\right) \chi_{2, s}\left(a_{2}+m \sqrt{D}\right)=$ $\chi_{1, s}^{n} \chi_{2, s}^{v(\alpha)-n}$.

(2ii) If $k<l, \Phi_{s}\left(\left(\begin{array}{cc}a_{1} & m \\ a_{1} \sqrt{D} & a_{2}+m \sqrt{D}\end{array}\right)\right)=\chi_{1, s}\left(\frac{a_{1} a_{2}}{a_{2}+m \sqrt{D}}\right) \chi_{2, s}\left(a_{2}+m \sqrt{D}\right)=$ $\chi_{1, s}^{v(\alpha)-k-\frac{1}{2}} \chi_{2, s}^{k+\frac{1}{2}}$

Proof One just need to use Lemma 4.2, and note that $v\left(a_{2}+m \sqrt{D}\right)=\operatorname{Min}\left\{l, k+\frac{1}{2}\right\}$, $v\left(a_{1} \sqrt{D}\right)=n+\frac{1}{2}$. Then the result is clear.

Now to compute $I\left(\alpha, f, \Phi_{s}\right)$ for this case, we compare Lemma 5.4 to Lemma 4.3. We see that the values of $\Phi_{s}$ differ by $\left(\frac{\chi_{2, s}}{\chi_{1, s}}\right)^{\frac{1}{2}}$ except the case (2i). Denote by $I$ the formula (4.10) for the inert case, and we get the relation

$$
\begin{aligned}
& I\left(\alpha, f, \Phi_{s}\right) \\
& \quad=\left(\frac{\chi_{2, s}}{\chi_{1, s}}\right)^{\frac{1}{2}}\left[I+\left(\left(\frac{\chi_{2, s}}{\chi_{1, s}}\right)^{-\frac{1}{2}}-1\right) \sum_{\frac{\nu(\alpha)}{2} \leq n \leq v(\alpha)} \sum_{\nu(\alpha)-n \leq k<\infty} \chi_{1, s}^{n} \chi_{2, s}^{\nu(\alpha)-n} q^{n-k}\left(1-q^{-1}\right)\right] .
\end{aligned}
$$

As a result,

$$
I\left(\alpha, f, \Phi_{s}\right)=\left(\frac{\chi_{2, s}}{\chi_{1, s}}\right)^{\frac{1}{2}} I+\left(1-\left(\frac{\chi_{2, s}}{\chi_{1, s}}\right)^{\frac{1}{2}}\right) \chi_{2, s}^{\nu(\alpha)} q^{-v(\alpha)} \frac{\left(q^{2} \frac{\chi_{1, s}}{\chi_{2, s}}\right)^{v(\alpha)-b}-\left(q^{2} \frac{\chi_{1, s}}{\chi_{2, s}}\right)^{v(\alpha)+1}}{1-q^{2} \frac{\chi_{1, s}}{\chi_{2, s}}}
$$

for $v(\alpha)=2 b, 2 b+1$.

Then one can follow the same steps to get:

$$
\mathbb{P}\left(s, w, f, \Phi_{s}\right)=\frac{\left(1+\mu_{1}\left(\chi_{1, s} \chi_{2, s}\right)^{\frac{1}{2}} \delta+\mu_{2}\left(\chi_{1, s} \chi_{2, s}\right)^{\frac{1}{2}} \delta+\delta^{2}\right)\left(1-q \delta^{2}\left(\frac{\chi_{2, s}}{\chi_{1, s}}\right)^{\frac{1}{2}}\right)}{\left(1-\mu_{1}^{2} \chi_{1, s} \chi_{2, s} \delta^{2}\right)\left(1-\mu_{2}^{2} \chi_{1, s} \chi_{2, s} \delta^{2}\right)\left(1-q \mu_{1} \chi_{1, s} \delta\right)\left(1-q \mu_{2} \chi_{1, s} \delta\right)},
$$

where $\delta=q^{-\left(\frac{w}{2}+\frac{1}{4}\right)}$. Recall that $\mu_{1} \mu_{2} \chi_{1} \chi_{2}=1$. When $w=\frac{1}{2}, \delta=q^{-\frac{1}{2}}$, so

$$
\begin{aligned}
\mathbb{P}\left(s, \frac{1}{2}, f, \Phi_{s}\right)= & \frac{\left(1+\left(\mu_{1}^{2} \chi_{1} \chi_{2}\right)\left(\varpi_{E}\right) q^{-\frac{1}{2}}\right)\left(1+\left(\mu_{2}^{2} \chi_{1} \chi_{2}\right)\left(\varpi_{E}\right) q^{-\frac{1}{2}}\right)}{\left(1-\mu_{1}^{2} \chi_{1} \chi_{2} q^{-1}\right)\left(1-\mu_{2}^{2} \chi_{1} \chi_{2} q^{-1}\right)} \\
& \times \frac{1-\frac{\chi_{1}}{\chi_{2}}\left(\varpi_{E}\right) q^{-(2 s+1)}}{\left(1-\mu_{1} \chi_{1} q^{-(2 s+1 / 2)}\right)\left(1-\mu_{2} \chi_{1} q^{-(2 s+1 / 2)}\right)} .
\end{aligned}
$$


This is exactly the expected $L$-factor

$$
\frac{L_{v}(\Pi \otimes \Omega, 1 / 2) L_{v}\left(\left.\pi \otimes \chi_{1}\right|_{\mathbb{F}^{*}}, 2 s+1 / 2\right)}{L_{v}(\eta, 1) L_{v}(\chi, 2 s+1)} .
$$

The local pairing $\left\langle F_{1}, F\right\rangle$ is the same as in the unramified case, as we choose same Schwartz function $f$, and the quadratic extension is not related to this calculation.

\subsection{Unramified special representation}

In this subsection we consider the case when $\pi=\sigma\left(\mu_{1}, \mu_{2}\right)$ is an unramified special representation. Then $\varphi$ and its corresponding Whittaker function $W_{\varphi}^{-}$belong to $\hat{\pi}=$ $\sigma\left(\mu_{1}^{-1}, \mu_{2}^{-1}\right)$. Since $\sigma\left(\mu_{1}^{-1}, \mu_{2}^{-1}\right)$ is equivalent to $\sigma\left(\mu_{2}^{-1}, \mu_{1}^{-1}\right)$, we can assume without loss of generality that $\sigma\left(\mu_{1}^{-1}, \mu_{2}^{-1}\right)$ is an irreducible subrepresentation of $\pi\left(\mu_{1}^{-1}, \mu_{2}^{-1}\right)$. This implies that $\mu_{1}^{-1} \mu_{2}=|\cdot|$. We shall do the computation in the split case, as the inert case would fail the Tunnell-Saito criterion as seen in Example 2.15. Write $\Phi_{s}=\Phi_{s}^{(1)} \Phi_{s}^{(2)}$ as in Sect. 4.2.

Pick

$$
f=\operatorname{char}\left(\left(\begin{array}{cc}
1 & 0 \\
\sqrt{D} & 1
\end{array}\right)\left(\begin{array}{cc}
O_{F} & O_{F} \\
\varpi O_{F} & O_{F}
\end{array}\right)\right) \times \operatorname{char}\left(O_{F}^{*}\right) .
$$

Pick $\Phi_{s}^{(2)}$ to be the unique right $K_{1}(\varpi)$-invariant function supported on $B K_{1}(\varpi)$ such that $\Phi_{s}^{(2)}(1)=1$, and $\Phi_{s}^{(1)}$ just to be the standard right $K$-invariant function. The property of $f$ implies that $F_{1}$ is the Gross-Prasad test vector while $F$ is the local newform, which is different from what we claimed in the beginning of this section. We shall now do a simple trick. Let

$$
f^{\prime}=\operatorname{char}\left(\left(\begin{array}{cc}
1 & 0 \\
\sqrt{D} & 1
\end{array}\right)\left(\begin{array}{cc}
O_{F} & O_{F} \\
\varpi O_{F} & O_{F}
\end{array}\right)\left(\begin{array}{cc}
1 & 0 \\
-\sqrt{D} & 1
\end{array}\right)\right) \times \operatorname{char}\left(O_{F}^{*}\right),
$$

and

$$
\Phi_{s}^{\prime}(g)=\Phi_{s}\left(g\left(\begin{array}{cc}
1 & 0 \\
-\sqrt{D} & 1
\end{array}\right)\right) .
$$

for $\Phi_{s}$ defined above. The matrix here should be thought of as an element of $\mathrm{GL}_{2}(\mathbb{F})$ diagonally embedded in $\mathrm{GL}_{2}(\mathbb{E})$.

By a simple change of variable, we have

$$
\mathbb{P}\left(s, w, f, \Phi_{s}\right)=\mathbb{P}\left(s, w, f^{\prime}, \Phi_{s}^{\prime}\right),
$$

and the Theta lifting of $f^{\prime}$ will be Gross-Prasad test vectors for both $F_{1}$ and $F$.

Proposition 5.5 Suppose that $\chi_{1}$ and $\chi_{2}$ are unramified, and $\mathbb{E} / \mathbb{F}$ is split. Suppose that $\pi=\sigma\left(\mu_{1}, \mu_{2}\right)$ is an unramified special representation such that $\mu_{1}^{-1} \mu_{2}=|\cdot|$. Further assume that 2 is a unit. Then

$$
\begin{aligned}
\mathbb{P}\left(s, w, f^{\prime}, \Phi_{s}^{\prime}\right) & =\mathbb{P}\left(s, w, f, \Phi_{s}\right) \\
& =\frac{1-q^{-1}}{(q+1)^{2}} \frac{1-\frac{\chi_{1, s}^{(1)}}{\left(1-\delta \chi_{2, s}^{(1)} \chi_{1, s}^{(2)} \mu_{2}\right)\left(1-\delta \chi_{1, s}^{(1)} \chi_{2, s}^{(2)} \mu_{2}\right)\left(1-q \delta \chi_{1, s}^{(1)} \chi_{1, s}^{(2)} \mu_{2}\right)} .}{} .
\end{aligned}
$$


At $w=1 / 2$, we have

$$
\begin{aligned}
& \mathbb{P}\left(s, 1 / 2, f^{\prime}, \Phi_{s}^{\prime}\right) \\
& \quad=\frac{1}{(q+1)^{2}} \frac{\left(1-q^{-1}\right)\left(1-\frac{\chi_{1}^{(1)}}{\chi_{2}^{(1)}} q^{-(2 s+1)}\right)}{\left(1-\mu_{2} \chi_{2}^{(1)} \chi_{1}^{(2)} q^{-1 / 2}\right)\left(1-\mu_{2} \chi_{1}^{(1)} \chi_{2}^{(2)} q^{-1 / 2}\right)\left(1-\mu_{2} \chi_{1}^{(1)} \chi_{1}^{(2)} q^{-(2 s+1 / 2)}\right)}
\end{aligned}
$$

The denominator of the expression is as expected, and

$$
\mathbb{P}^{0}\left(s, 1 / 2, f^{\prime}, \Phi_{s}^{\prime}\right)=\frac{1}{q\left(1-\chi^{(2)} q^{-(2 s+1)}\right)} .
$$

We first work out the Whittaker function $W_{\varphi}^{-}$for a new form $\varphi$ in $\hat{\pi}$. It's a classical result that such $\varphi$ should be $K_{1}(\varpi)$-invariant, and up to a constant multiple

$$
\left.\varphi\right|_{K}=\operatorname{char}\left(B\left(O_{F}\right) K_{1}(\varpi)\right)-q^{-1} \operatorname{char}\left(B\left(O_{F}\right)\left(\begin{array}{ll}
1 & 0 \\
1 & 1
\end{array}\right) K_{1}(\varpi)\right) \text {. }
$$

The corresponding $W_{\varphi}^{-}$is also $K_{1}(\varpi)$-invariant. According to Lemma 2.1, we just need to figure out $W_{\varphi}^{-}\left(\left(\begin{array}{ll}\alpha & 0 \\ 0 & 1\end{array}\right)\right)$ and $W_{\varphi}^{-}\left(\left(\begin{array}{ll}\alpha & 0 \\ 0 & 1\end{array}\right)\left(\begin{array}{ll}1 & 0 \\ 1 & 1\end{array}\right)\right)$. It is possible to obtain these values directly from classical theories, but we will start with a more general setting, as it will be helpful for later cases. Recall that $W_{\varphi}^{-}$is the Whittaker funciton associated to $\psi^{-}$. So

$$
W_{\varphi}^{-}(g)=\int_{m \in \mathbb{F}} \varphi\left(\omega\left(\begin{array}{cc}
1 & m \\
0 & 1
\end{array}\right) g\right) \psi(m) d m
$$

In general we want to write

$$
\omega\left(\begin{array}{cc}
1 & m \\
0 & 1
\end{array}\right)\left(\begin{array}{ll}
\alpha & 0 \\
0 & 1
\end{array}\right)\left(\begin{array}{cc}
1 & 0 \\
\varpi^{j} & 1
\end{array}\right)=\left(\begin{array}{cc}
\varpi^{j} & 1 \\
-\alpha-m \varpi^{j} & -m
\end{array}\right)
$$

in form of $B\left(\begin{array}{cc}1 & 0 \\ \varpi^{i} & 1\end{array}\right) K_{1}\left(\varpi^{c}\right)$ for $0 \leq i, j \leq c$. Note that if $i=c$, then $\left(\begin{array}{cc}1 & 0 \\ \varpi^{i} & 1\end{array}\right)$ is absorbed into $K_{1}\left(\varpi^{c}\right)$. Same for $\mathbf{j}$.

Lemma 5.6 (1) Suppose that $i=0$.

(1i) If $j=0$, we need $m \notin \alpha\left(-1+\varpi O_{F}\right)$ for $\left(\begin{array}{cc}\varpi^{j} & 1 \\ -\alpha-m \varpi^{j} & -m\end{array}\right) \in$

$$
B\left(\begin{array}{cc}
1 & 0 \\
\varpi^{i} & 1
\end{array}\right) K_{1}\left(\varpi^{c}\right)
$$

(1ii) If $j>0$, we need $v(m) \geq v(\alpha)$.

Under the above conditions we can write $\left(\begin{array}{cc}\varpi^{j} & 1 \\ -\alpha-m \varpi^{j} & -m\end{array}\right)$ as

$$
\left(\begin{array}{cc}
-\frac{\alpha}{\alpha+m \varpi^{j}} & \varpi^{j}+\frac{\alpha}{\alpha+m \varpi^{j}} \\
0 & -\alpha-m \varpi^{j}
\end{array}\right)\left(\begin{array}{ll}
1 & 0 \\
1 & 1
\end{array}\right)\left(\begin{array}{cc}
1 & -1+\frac{m}{\alpha+m \varpi^{j}} \\
0 & 1
\end{array}\right)
$$

(2) Suppose that $i=c$.

(2i) If $j<c$, we need $m \in \alpha \varpi^{-j}\left(-1+\varpi^{c-j} O_{F}\right)$; 
(2ii) If $j=c$, we need $v(m) \leq v(\alpha)-c$.

Under the above conditions, we can write $\left(\begin{array}{cc}\varpi^{j} & 1 \\ -\alpha-m \varpi^{j} & -m\end{array}\right)$ as

$$
\left(\begin{array}{cc}
-\frac{\alpha}{m} & 1 \\
0 & -m
\end{array}\right)\left(\begin{array}{cc}
1 & 0 \\
\frac{\alpha}{m}+\varpi^{j} & 1
\end{array}\right)
$$

(3) Suppose that $0<i<c$.

(3i) If $j<i$, we need $m \in \alpha \varpi^{-j}\left(-1+\varpi^{i-j} O_{F}^{*}\right)$;

(3ii) If $j>i$, we need $v(m)=v(\alpha)-i$;

(3iii) If $j=i$, we need $v(m) \leq v(\alpha)-i$ but $m \notin \alpha \varpi^{-i}\left(-1+\varpi O_{F}\right)$.

Under the above conditions we can write $\left(\begin{array}{cc}\varpi^{j} & 1 \\ -\alpha-m \varpi^{j} & -m\end{array}\right)$ as

$$
\left(\begin{array}{cc}
-\frac{\alpha \varpi^{i}}{\alpha+m \varpi^{j}} & 1 \\
0 & -m
\end{array}\right)\left(\begin{array}{cc}
1 & 0 \\
\varpi^{i} & 1
\end{array}\right)\left(\begin{array}{cc}
\frac{\alpha+m \varpi^{j}}{m \varpi^{i}} & 0 \\
0 & 1
\end{array}\right)
$$

This lemma is straightforward to check. We will leave the proof to the reader.

Corollary 5.7 Assume that $\mu_{1}$ and $\mu_{2}$ are unramified and $\varphi \in \sigma\left(\mu_{1}^{-1}, \mu_{2}^{-1}\right)$ is given by (5.14). Let $W_{\varphi}^{-}$be the normalized Whittaker function associated to $\varphi$. Then

$$
W_{\varphi}^{-}\left(\left(\begin{array}{ll}
\alpha & 0 \\
0 & 1
\end{array}\right)\right)= \begin{cases}\mu_{1}^{-v(\alpha)} q^{-v(\alpha) / 2}, & \text { if } \nu(\alpha) \geq 0 \\
0, & \text { if } \nu(\alpha)<0\end{cases}
$$

and

$$
W_{\varphi}^{-}\left(\left(\begin{array}{ll}
\alpha & 0 \\
0 & 1
\end{array}\right)\left(\begin{array}{ll}
1 & 0 \\
1 & 1
\end{array}\right)\right)= \begin{cases}-q^{-1} \mu_{1}^{-v(\alpha)} q^{-v(\alpha) / 2} \psi(-\alpha), & \text { if } v(\alpha) \geq-1 \\
0, & \text { if } v(\alpha)<-1 .\end{cases}
$$

Proof Put $c=1$, and consider $j=1$. By formula (5.14) and (1ii) (2ii) of the above lemma:

$$
\begin{aligned}
W_{\varphi}^{-}\left(\left(\begin{array}{cc}
\alpha & 0 \\
0 & 1
\end{array}\right)\right)= & \int_{m \in \mathbb{F}} \varphi\left(\omega\left(\begin{array}{cc}
1 & m \\
0 & 1
\end{array}\right)\left(\begin{array}{cc}
\alpha & 0 \\
0 & 1
\end{array}\right)\right) \psi(m) d m \\
= & \int_{\nu(m) \leq \nu(\alpha)-1} \mu_{1}^{-1}\left(-\frac{\alpha}{m}\right) \mu_{2}^{-1}(-m)\left|\frac{\alpha}{m^{2}}\right|^{1 / 2} \psi(m) d m-q^{-1} \\
& \times \int_{\nu(m) \geq v(\alpha)} \mu_{1}^{-1}(-1) \mu_{2}^{-1}(-\alpha)\left|\frac{1}{\alpha}\right|^{1 / 2} \psi(m) d m \\
= & \left\{\begin{array}{l}
\left(-q^{-1}-q^{-2}\right) \mu_{1}^{-v(\alpha)} q^{-\nu(\alpha) / 2}, \text { if } v(\alpha) \geq 0 \\
0, \text { if } v(\alpha)<0 .
\end{array}\right.
\end{aligned}
$$

In the last equation, one need to use Lemma 2.3 and that $\mu_{1}^{-1} \mu_{2}=|\cdot|$.

If we normalize $W_{\varphi}^{-}\left(\left(\begin{array}{ll}1 & 0 \\ 0 & 1\end{array}\right)\right)$ to be 1 , then

$$
W_{\varphi}^{-}\left(\left(\begin{array}{ll}
\alpha & 0 \\
0 & 1
\end{array}\right)\right)= \begin{cases}\mu_{1}^{-v(\alpha)} q^{-v(\alpha) / 2}, & \text { if } v(\alpha) \geq 0 \\
0, & \text { if } v(\alpha)<0\end{cases}
$$


Similarly, we consider the case when $j=0$. From (1i) and (2i):

$$
\begin{aligned}
W_{\varphi}^{-}\left(\left(\begin{array}{ll}
\alpha & 0 \\
0 & 1
\end{array}\right)\left(\begin{array}{ll}
1 & 0 \\
1 & 1
\end{array}\right)\right)= & \int_{m \in \mathbb{F}} \varphi\left(\omega\left(\begin{array}{cc}
1 & m \\
0 & 1
\end{array}\right)\left(\begin{array}{ll}
\alpha & 0 \\
0 & 1
\end{array}\right)\left(\begin{array}{ll}
1 & 0 \\
1 & 1
\end{array}\right)\right) \psi(m) d m \\
= & \int_{m \in \alpha\left(-1+\varpi O_{F}\right)} \mu_{1}^{-1}\left(-\frac{\alpha}{m}\right) \mu_{2}^{-1}(-m)\left|\frac{\alpha}{m^{2}}\right|^{1 / 2} \psi(m) d m \\
& -q^{-1} \int^{m \notin \alpha\left(-1+\varpi O_{F}\right)} \mu_{1}^{-1}\left(-\frac{\alpha}{\alpha+m}\right) \mu_{2}^{-1}(-\alpha-m) \\
& \times\left|\frac{\alpha}{(\alpha+m)^{2}}\right|^{1 / 2} \psi(m) d m \\
= & \begin{cases}q^{-1}\left(q^{-1}+q^{-2}\right) \mu_{1}^{-v(\alpha)} q^{-v(\alpha) / 2}, & \text { if } v(\alpha) \geq 0 ; \\
q^{-1}\left(1+q^{-1}\right) \mu_{1} q^{-1 / 2} \psi(-\alpha), & \text { if } v(\alpha)=-1 ; \\
0, & \text { if } v(\alpha)<-1 .\end{cases}
\end{aligned}
$$

After normalization, we get

$$
W_{\varphi}^{-}\left(\left(\begin{array}{ll}
\alpha & 0 \\
0 & 1
\end{array}\right)\left(\begin{array}{ll}
1 & 0 \\
1 & 1
\end{array}\right)\right)= \begin{cases}-q^{-1} \mu_{1}^{-v(\alpha)} q^{-v(\alpha) / 2} \psi(-\alpha), & \text { if } v(\alpha) \geq-1 \\
0, & \text { if } v(\alpha)<-1\end{cases}
$$

Suppose that the chosen Schwartz function $f$ is also $K_{1}(\varpi)$-invariant under the Weil representation $r^{\prime}$. Then by Lemma 2.2 and Corollary 5.7, we have:

$$
\begin{aligned}
\mathbb{P}\left(s, w, f, \Phi_{s}\right)= & \frac{1}{q+1} \int_{v(\alpha) \geq 0} \mu_{1}^{-v(\alpha)} q^{-(w / 2+1 / 4) v(\alpha)} \Phi_{s}(\alpha)^{-1} I\left(\alpha, f, \Phi_{s}\right) d^{*} \alpha \\
& +\frac{q}{q+1} \int_{\nu(\alpha) \geq-1}-q^{-1} \mu_{1}^{-v(\alpha)} \psi(-\alpha) q^{-(w / 2+1 / 4) v(\alpha)} \Phi_{s}(\alpha)^{-1} \\
& \times I\left(\alpha, r^{\prime}\left(\left(\begin{array}{ll}
1 & 0 \\
1 & 1
\end{array}\right)\right) f, \Phi_{s}\right) d^{*} \alpha .
\end{aligned}
$$

Now we verify the $K_{1}(\varpi)$-invariance of $f$ and calculate $I\left(\alpha, f, \Phi_{s}\right)$ and $I\left(\alpha, r^{\prime}\left(\left(\begin{array}{ll}1 & 0 \\ 1 & 1\end{array}\right)\right) f, \Phi_{s}\right)$. Recall that we picked

$$
f=\operatorname{char}\left(\left(\begin{array}{cc}
1 & 0 \\
\sqrt{D} & 1
\end{array}\right)\left(\begin{array}{cc}
O_{F} & O_{F} \\
\varpi O_{F} & O_{F}
\end{array}\right)\right) \times \operatorname{char}\left(O_{F}^{*}\right)
$$

It's clearly right $K_{1}(\varpi)$-invariant. This choice of Schwartz function is motivated by Example 2.19. It can also be written as

$$
f=\sum_{a_{0} \in O_{F} / \varpi O_{F}} \operatorname{char}\left(\left(\begin{array}{cc}
a_{0}+\varpi O_{F} & O_{F} \\
a_{0} \sqrt{D}+\varpi O_{F} & O_{F}
\end{array}\right)\right) \times \operatorname{char}\left(O_{F}^{*}\right) .
$$


Using Lemmas (2.10) and (2.13) one can easily check that $f$ is $K_{1}(\varpi)$-invariant under the Weil representation, and

$$
\begin{aligned}
r^{\prime}\left(\left(\begin{array}{ll}
1 & 0 \\
1 & 1
\end{array}\right)\right) f= & q^{-2} \sum_{a_{0} \in O_{F} / \varpi O_{F}} \psi\left(u\left[\left(x_{1}-a_{0}\right) x_{4}-x_{2}\left(x_{3}-a_{0} \sqrt{D}\right)\right]\right) \\
& \times \operatorname{char}\left(\left(\begin{array}{ll}
O_{F} & \varpi^{-1} O_{F} \\
O_{F} & \varpi^{-1} O_{F}
\end{array}\right)\right) \times \operatorname{char}\left(O_{F}^{*}\right) .
\end{aligned}
$$

This sum is also right $K_{1}(\varpi)$-invariant.

Recall that $\Phi_{s}^{(2)}$ is the unique right $K_{1}(\varpi)$-invariant function supported on $B K_{1}(\varpi)$, and $\Phi_{s}^{(1)}$ is the standard right $K$-invariant function.

We will write $\mathrm{GL}_{2}(\mathbb{F})=\left(\begin{array}{cc}1 & 0 \\ \sqrt{D} & 1\end{array}\right) B K_{1}(\varpi) \cup\left(\begin{array}{cc}1 & 0 \\ \sqrt{D} & 1\end{array}\right) B\left(\begin{array}{ll}1 & 0 \\ 1 & 1\end{array}\right) K_{1}(\varpi)$. The matrix $\left(\begin{array}{cc}1 & 0 \\ \sqrt{D} & 1\end{array}\right)$ on the left amounts to a change of variable. Then by our choice of $\Phi_{s}^{(2)}$, in particular its support, we only need to integrate over $\left(\begin{array}{cc}1 & 0 \\ \sqrt{D} & 1\end{array}\right) B K_{1}(\varpi)$ for $I\left(\alpha, f, \Phi_{s}\right)$ and $I\left(\alpha, r^{\prime}\left(\left(\begin{array}{ll}1 & 0 \\ 1 & 1\end{array}\right)\right) f, \Phi_{s}\right)$. By the right $K_{1}(\varpi)$-invariance of $\Phi_{s}$, we can write

$$
\begin{aligned}
I\left(\alpha, f, \Phi_{s}\right)= & \frac{1}{q+1} \int_{\substack{a_{1}, a_{2}, m \in O_{F} \\
v\left(a_{1}\right)+v\left(a_{2}\right)=v(\alpha)}} \Phi_{s}^{(1)}\left(\left(\begin{array}{cc}
1 & 0 \\
2 \sqrt{D} & 1
\end{array}\right)\left(\begin{array}{cc}
a_{1} & m \\
0 & a_{2}
\end{array}\right)\right) \\
& \times \Phi_{s}^{(2)}\left(\left(\begin{array}{cc}
a_{1} & m \\
0 & a_{2}
\end{array}\right)\right) d m d^{*} a_{2}\left|a_{1}\right|^{-1} d^{*} a_{1} .
\end{aligned}
$$

Note that the domain and the integrand of this integral is exactly the same as (4.13) in Sect. 4.2. Denote by $I$ the result we got in (4.17). Then

$$
I\left(\alpha, f, \Phi_{s}\right)=\frac{1}{q+1} I
$$

Now we consider $I\left(\alpha, r^{\prime}\left(\left(\begin{array}{ll}1 & 0 \\ 1 & 1\end{array}\right)\right) f, \Phi_{s}\right)$. For the matrix $\left(\begin{array}{cc}1 & 0 \\ \sqrt{D} & 1\end{array}\right)\left(\begin{array}{cc}a_{1} & m \\ 0 & a_{2}\end{array}\right)=$ $\left(\begin{array}{cc}a_{1} & m \\ a_{1} \sqrt{D} & a_{2}+m \sqrt{D}\end{array}\right)$

$$
\psi\left(u\left[\left(x_{1}-a_{0}\right) x_{4}-x_{2}\left(x_{3}-a_{0} \sqrt{D}\right)\right]\right)=\psi\left(\alpha\left(1-\frac{a_{0}}{a_{1}}\right)\right) .
$$

Here we have used $u=\frac{\alpha}{\operatorname{det} x} \operatorname{in} I\left(\alpha, r^{\prime}\left(\left(\begin{array}{ll}1 & 0 \\ 1 & 1\end{array}\right)\right) f, \Phi_{s}\right)$. Then by (5.23), 


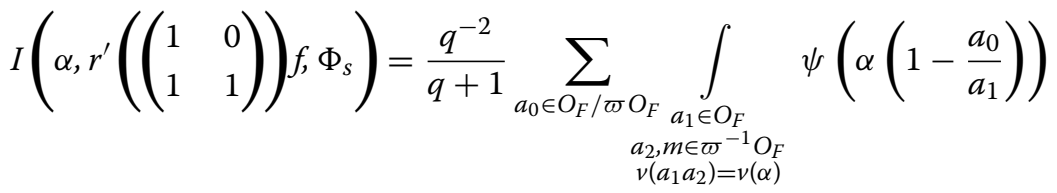

$$
\begin{aligned}
& \times \Phi_{s}^{(1)}\left(\left(\begin{array}{cc}
a_{1} & m \\
2 a_{1} \sqrt{D} & a_{2}+2 m \sqrt{D}
\end{array}\right)\right) \\
& \times \Phi_{s}^{(2)}\left(\left(\begin{array}{cc}
a_{1} & m \\
0 & a_{2}
\end{array}\right)\right) d m d^{*} a_{2}\left|a_{1}\right|^{-1} d^{*} a_{1} \text {. }
\end{aligned}
$$

Compare the domain of each integral in this expression with the domain of (4.13), we note that we have two additional parts:

$$
\left\{v\left(a_{1}\right), v\left(a_{2}\right) \geq 0, v(m)=-1\right\} \quad \text { and } \quad\left\{v\left(a_{1}\right)=v(\alpha)+1, v\left(a_{2}\right)=-1, v(m) \geq-1\right\} .
$$

Also note $\psi\left(\alpha\left(1-\frac{a_{0}}{a_{1}}\right)\right)=1$ if $v(\alpha) \geq 0$ and $v\left(a_{1}\right) \leq v(\alpha)$. So over the common domain $\left\{a_{1}, m, a_{2} \in O_{F}\right\}$, the integral gives $I$ as in (4.17). It's not difficult to work out the integral over the two additional parts. Then one can get

$$
\begin{aligned}
& I\left(\alpha, r^{\prime}\left(\left(\begin{array}{ll}
1 & 0 \\
1 & 1
\end{array}\right)\right) f, \Phi_{s}\right) \\
& \quad= \begin{cases}\frac{q^{-1}}{q+1}\left(I+(q-1) \frac{\chi_{1, s}^{(1)}}{\chi_{2, s}^{(1)}}\left(\chi_{1, s}^{(1)} \chi_{2, s}^{(2)}\right)^{v(\alpha)} \frac{1-\left(\frac{q x_{1, s}^{(2)}}{\chi_{2, s}^{(2)}}\right)^{v(\alpha)+1}}{1-\frac{q \chi_{1, s}^{(2)}}{\chi_{2, s}^{(2)}}}\right), & \text { if } v(\alpha) \geq 0 ; \\
0, & \text { otherwise. }\end{cases}
\end{aligned}
$$

By combining (5.25) (5.28) with (5.21), we get

$$
\mathbb{P}\left(s, w, f, \Phi_{s}\right)=\frac{1-q^{-1}}{(q+1)^{2}} \frac{1-\frac{\chi_{1, s}^{(1)}}{\chi_{2, s}^{(1)}}}{\left(1-\delta \chi_{2, s}^{(1)} \chi_{1, s}^{(2)} \mu_{2}\right)\left(1-\delta \chi_{1, s}^{(1)} \chi_{2, s}^{(2)} \mu_{2}\right)\left(1-q \delta \chi_{1, s}^{(1)} \chi_{1, s}^{(2)} \mu_{2}\right)} .
$$

At $w=1 / 2$, we have

$$
\begin{aligned}
& \mathbb{P}\left(s, 1 / 2, f, \Phi_{s}\right) \\
& \quad=\frac{1}{(q+1)^{2}} \frac{\left(1-q^{-1}\right)\left(1-\frac{\chi_{1}^{(1)}}{\chi_{2}^{(1)}} q^{-(2 s+1)}\right)}{\left(1-\mu_{2} \chi_{2}^{(1)} \chi_{1}^{(2)} q^{-1 / 2}\right)\left(1-\mu_{2} \chi_{1}^{(1)} \chi_{2}^{(2)} q^{-1 / 2}\right)\left(1-\mu_{2} \chi_{1}^{(1)} \chi_{1}^{(2)} q^{-(2 s+1 / 2)}\right)}
\end{aligned}
$$

Now we evaluate local pairing $\left\langle F_{1}, F\right\rangle$ for $f^{\prime}$ by (2.27).

Let

$$
f^{\prime \prime}=\operatorname{char}\left(\left(\begin{array}{cc}
O_{F} & O_{F} \\
\varpi O_{F} & O_{F}
\end{array}\right)\right) \times \operatorname{char}\left(O_{F}^{*}\right) .
$$

It differs from $f^{\prime}$ by conjugation, but conjugation acts trivially on the center. So

$$
\begin{aligned}
\left\langle F_{1}, F\right\rangle & =\int_{N Z \backslash \mathrm{GL}_{2}\left(\mathbb{F}_{v}\right)} \int_{\mathbb{F}_{v}^{*}} W_{\varphi}^{-}(h) r^{\prime}(h) f^{\prime}\left(x, x^{-2}\right) w_{\hat{\pi}}(x) d^{*} x d h \\
& =\int_{N Z \backslash \mathrm{GL}_{2}\left(\mathbb{F}_{v}\right)} \int_{\mathbb{F}_{v}^{*}} W_{\varphi}^{-}(h) r^{\prime}(h) f^{\prime \prime}\left(x, x^{-2}\right) w_{\hat{\pi}}(x) d^{*} x d h .
\end{aligned}
$$


The latter is easier to compute, and one can easily check that

$$
\begin{aligned}
\left\langle F_{1}, F\right\rangle & =\frac{1}{q+1} \sum_{n \geq 0} \mu_{1}^{-2 n} q^{-n}\left(\mu_{1} \mu_{2}\right)^{n}-\frac{q^{-1}}{q+1} \sum_{n \geq 0} \mu_{1}^{-2 n} q^{-n}\left(\mu_{1} \mu_{2}\right)^{n} \\
& =\frac{q}{(q+1)^{2}} .
\end{aligned}
$$

Here we have used that $\mu_{1}^{-1} \mu_{2}=|\cdot|$.

The expected L-factors in this case is

$$
\begin{aligned}
& \quad \frac{\zeta(2)}{L(\pi, A d, 1)} \frac{L(\Pi \otimes \Omega, 1 / 2) L\left(\left.\pi \otimes \chi_{1}\right|_{\mathbb{F}^{*}}, 2 s+1 / 2\right)}{L(\eta, 1) L(\chi, 2 s+1)} \\
& \quad=\frac{\left(1-q^{-1}\right)\left(1-\chi^{(1)} q^{-(2 s+1)}\right)\left(1-\chi^{(2)} q^{-(2 s+1)}\right)}{\left(1-\mu_{2} \chi_{2}^{(1)} \chi_{1}^{(2)} q^{-1 / 2}\right)\left(1-\mu_{2} \chi_{1}^{(1)} \chi_{2}^{(2)} q^{-1 / 2}\right)\left(1-\mu_{2} \chi_{1}^{(1)} \chi_{1}^{(2)} q^{-(2 s+1 / 2)}\right)}
\end{aligned}
$$

Thus

$$
\mathbb{P}^{0}\left(s, 1 / 2, f^{\prime}, \Phi_{s}^{\prime}\right)=\frac{1}{q\left(1-\chi^{(2)} q^{-(2 s+1)}\right)} .
$$

$5.3 \pi$ of higher level and $\mathbb{E} / \mathbb{F}$ split

Definition 5.8 Let

$$
\begin{aligned}
& f=\operatorname{char}\left(\left(\begin{array}{cc}
1 & 0 \\
\sqrt{D} & 1
\end{array}\right)\left(\begin{array}{cc}
O_{F} & O_{F} \\
\varpi^{c} O_{F} & O_{F}
\end{array}\right)\right) \times \operatorname{char}\left(O_{F}^{*}\right) \\
& f^{\prime}=\operatorname{char}\left(\left(\begin{array}{cc}
1 & 0 \\
\sqrt{D} & 1
\end{array}\right)\left(\begin{array}{cc}
O_{F} & O_{F} \\
\varpi^{c} O_{F} & O_{F}
\end{array}\right)\left(\begin{array}{cc}
1 & 0 \\
-\sqrt{D} & 1
\end{array}\right)\right) \times \operatorname{char}\left(O_{F}^{*}\right) .
\end{aligned}
$$

Pick $\Phi_{s}^{(2)}$ to be the unique right $K_{1}\left(\varpi^{c}\right)$-invariant function supported on $B K_{1}\left(\varpi^{c}\right)$ such that $\Phi_{s}^{(2)}(1)=1$, and $\Phi_{s}^{(1)}$ to be the standard right $K$-invariant function. For $\Phi_{s}=\Phi_{s}^{(1)} \Phi_{s}^{(2)}$, let

$$
\Phi_{s}^{\prime}(g)=\Phi_{s}\left(g\left(\begin{array}{cc}
1 & 0 \\
-\sqrt{D} & 1
\end{array}\right)\right)
$$

In this subsection we will prove:

Proposition 5.9 Suppose that $\pi$ is a representation of level $c>1$ with unramified central character. Suppose that $\chi_{1}$ and $\chi_{2}$ are unramified and $\mathbb{E} / \mathbb{F}$ is split. Further suppose that 2 is a unit.

Then

$$
\mathbb{P}\left(s, w, f^{\prime}, \Phi_{s}^{\prime}\right)=\mathbb{P}\left(s, w, f, \Phi_{s}\right)=\frac{1-q^{-1}}{(q+1)^{2} q^{2 c-2}}\left(1-\frac{\chi_{1}^{(1)}}{\chi_{2}^{(1)}} q^{-(2 s+1)}\right) .
$$

Apart from the constant term, the denominator is the same as $L_{\nu}(\Pi \otimes \Omega, 1 / 2) L_{v}(\pi \otimes$ $\left.\left.\chi_{1}\right|_{\mathbb{F}^{*}}, 2 s+1 / 2\right)$, which is 1 in this case. Then

$$
\mathbb{P}^{0}\left(s, 1 / 2, f^{\prime}, \Phi_{s}^{\prime}\right)=\frac{1}{q^{c}} \frac{L(\pi, A d, 1)}{1-\chi^{(2)} q^{-(2 s+1)}} .
$$




\subsubsection{Supercuspidal representations}

We first assume that $\pi$ and $\hat{\pi}$ are supercuspidal representations. Then it is easier to describe the group actions using the Kirillov model.

For basic properties of the Kirillov model, one can read [15]. For the level and the new form of the Kirillov model of a supercuspidal representation, we mainly follow [5]. Here we just recount part of the facts necessary for our computations.

For a fixed additive character $\psi^{-}$, the Kirillov model of $\hat{\pi}$ is a unique realization on the space of Schwartz functions $S\left(\mathbb{F}^{*}\right)$ such that

$$
\hat{\pi}\left(\left(\begin{array}{cc}
a_{1} & m \\
0 & a_{2}
\end{array}\right)\right) \varphi(x)=w_{\hat{\pi}}\left(a_{2}\right) \psi\left(-m a_{2}^{-1} x\right) \varphi\left(a_{1} a_{2}^{-1} x\right),
$$

where $w_{\hat{\pi}}$ is the central character for $\hat{\pi}$. By Bruhat decomposition, one just has to know the action of $\omega=\left(\begin{array}{cc}0 & 1 \\ -1 & 0\end{array}\right)$ to understand the whole group action.

Define

$$
\mathbf{1}_{v, n}(x)= \begin{cases}v(u), & \text { if } x=u \varpi^{n} \text { for } u \in O_{F}^{*} \\ 0, & \text { otherwise. }\end{cases}
$$

Roughly speaking, it's the character $v$ supported at $v(x)=n$. Such functions provide a basis for $S\left(\mathbb{F}^{*}\right)$. We can then describe the action of $\omega=\left(\begin{array}{cc}0 & 1 \\ -1 & 0\end{array}\right)$ on $\mathbf{1}_{v, n}$ explicitly according to [15]:

$$
\hat{\pi}(\omega) \mathbf{1}_{v, n}=C_{v w_{0}^{-1}} z_{0}^{-n} \mathbf{1}_{v^{-1} w_{0},-n+n_{v-1}} .
$$

Here $z_{0}=w_{\hat{\pi}}(\varpi)$ and $w_{0}=\left.w_{\hat{\pi}}\right|_{O_{F}^{*}} \cdot n_{v}$ is an integer. $C_{v}$ and $n_{v}$ are decided by the representation $\hat{\pi}$ and the character $v$ (and independent of $n$ ).

The relation $\omega^{2}=-\left(\begin{array}{ll}1 & 0 \\ 0 & 1\end{array}\right)$ implies that

$$
n_{v}=n_{v^{-1} w_{0}^{-1}}, C_{v} C_{v^{-1} w_{0}^{-1}}=w_{0}(-1) z_{0}^{n_{v}} .
$$

It's well-known that

$$
n_{v} \leq-2
$$

for any $v$. According to Proposition A.1, when the supercuspidal representation is fixed with the central character unramified, $n_{v}$ only depends on the level of $v$. When we pick $v$ to be the trivial character, the number $-n_{1}$ is actually the level of this supercuspidal representation, that is

$$
c=-n_{1} .
$$

The argument in [5] with slight modification can show that there is a unique up to constant element $\varphi$ in the supercupidal representation which is invariant under $K_{1}\left(\varpi^{c}\right)$. One can easily check that $\varphi=\mathbf{1}_{1,0}$ is such an element.

From now on, we assume that the central character $w_{\hat{\pi}}$ is unramified, so $w_{0}=\left.w_{\hat{\pi}}\right|_{O_{F}^{*}}=$ 1. For the newform $\varphi=\mathbf{1}_{1,0}$, its associated Whittaker function $W_{\varphi}^{-}$is also right $K_{1}\left(\varpi^{c}\right)$ invariant. We can calculate $W_{\varphi}^{-}$according to the relation between the Kirillov model and the Whittaker model:

$$
W_{\varphi}^{-}\left(\left(\begin{array}{ll}
\alpha & 0 \\
0 & 1
\end{array}\right)\left(\begin{array}{cc}
1 & 0 \\
\varpi^{i} & 1
\end{array}\right)\right)=\hat{\pi}\left(\left(\begin{array}{cc}
1 & 0 \\
\varpi^{i} & 1
\end{array}\right)\right) \varphi(\alpha) .
$$


It's difficult to describe $W_{\varphi}^{-}$explicitly. But it will suffice to know only some specific integrals for $W_{\varphi}^{-}\left(\left(\begin{array}{ll}\alpha & 0 \\ 0 & 1\end{array}\right)\left(\begin{array}{cc}1 & 0 \\ \varpi^{i} & 1\end{array}\right)\right)$.

Lemma 5.10 Suppose that $\hat{\pi}$ is a supercuspidal representation with unramified central character. Let $W_{\varphi}^{-}$be the Whittaker function associated to $\varphi=1_{1,0} \in \hat{\pi}$.

(1) $W_{\varphi}^{-}\left(\left(\begin{array}{ll}\alpha & 0 \\ 0 & 1\end{array}\right)\right)=1_{1,0}(\alpha)$. For $0 \leq i<c, W_{\varphi}^{-}\left(\left(\begin{array}{cc}\alpha & 0 \\ 0 & 1\end{array}\right)\left(\begin{array}{cc}1 & 0 \\ \varpi^{i} & 1\end{array}\right)\right)$ is supported only at $v(\alpha)=\min \{0,2 i-c\}$.

(2)

$\int_{v(\alpha)=\min \{0,2 i-c\}} W_{\varphi}^{-}\left(\left(\begin{array}{ll}\alpha & 0 \\ 0 & 1\end{array}\right)\left(\begin{array}{cc}1 & 0 \\ \varpi^{i} & 1\end{array}\right)\right) d^{*} \alpha= \begin{cases}1, & \text { if } i \geq c ; \\ -\frac{1}{q-1}, & \text { if } i=c-1 \\ 0, & \text { otherwise. }\end{cases}$

$$
\int_{v(\alpha)=\min \{0,2 i-c\}} W_{\varphi}^{-}\left(\left(\begin{array}{cc}
\alpha & 0 \\
0 & 1
\end{array}\right)\left(\begin{array}{cc}
1 & 0 \\
\varpi^{i} & 1
\end{array}\right)\right) \psi\left(\varpi^{-i} \alpha\right) d^{*} \alpha= \begin{cases}C_{1}, & \text { if } i=0 ; \\
-\frac{1}{q-1} C_{1} w_{\hat{\pi}}, & \text { if } i=1 ; \\
0, & \text { otherwise. }\end{cases}
$$

Proof The first statement of (1) is clear. Now let $0 \leq i<c$. According to Proposition A.1, if $v$ is a character of level $i$, then $n_{v}=\min \left\{n_{1},-2 i\right\}$. Note that

$$
\begin{aligned}
& \left(\begin{array}{cc}
1 & 0 \\
\varpi^{i} & 1
\end{array}\right)=-\omega\left(\begin{array}{cc}
1 & -\varpi^{i} \\
0 & 1
\end{array}\right) \omega, \\
& \hat{\pi}(\omega) \mathbf{1}_{1,0}=C_{w_{0}^{-1}} \mathbf{1}_{w_{0}, n_{1}}=C_{1} \mathbf{1}_{1, n_{1}} .
\end{aligned}
$$

The action of $\left(\begin{array}{cc}1 & -\varpi^{i} \\ 0 & 1\end{array}\right)$ for $i<c$ will give a non-trivial factor $\psi\left(\varpi^{i} x\right)$ at $v(x)=n_{1}$. By the classical result about Gauss sum, $\hat{\pi}\left(\left(\begin{array}{cc}1 & -\varpi^{i} \\ 0 & 1\end{array}\right) \omega\right) \mathbf{1}_{1,0}$ is a linear combination of all characters of level $-n_{1}-i=c-i$, supported at $v(x)=n_{1}$. (It should be understood that if $c-i=1$, then this is a linear combination of all characters of level 1 and 0 .) After another action of $\omega$ their levels will not be changed, but supported at

$$
v(x)=-n_{1}+\min \left\{n_{1},-2(c-i)\right\}=\min \{0,2 i-c\} .
$$

This finishes the proof of part (1).

When we integrate $W_{\varphi}^{-}\left(\left(\begin{array}{ll}\alpha & 0 \\ 0 & 1\end{array}\right)\left(\begin{array}{cc}1 & 0 \\ \varpi^{i} & 1\end{array}\right)\right)$ in $\alpha$, we are just finding the level 0 component of it. By the discussion above, this is only possible when $i=c$ or $c-1$. The integral in the case $i=c$ is obvious. When $i=c-1$, one can compute that the level 0 component of $\hat{\pi}\left(\left(\begin{array}{cc}1 & -\varpi^{i} \\ 0 & 1\end{array}\right) \omega\right) \mathbf{1}_{1,0}$ is

$$
-\frac{1}{q-1} C_{1} \mathbf{1}_{1, n_{1}}
$$

as $\int_{x \in \varpi^{-1} O_{F}^{*}} \psi(x) d^{*} x=-\frac{1}{q-1}$. Then the action of $\omega$ will map it to $-\frac{1}{q-1} \mathbf{1}_{1,0}$, using (5.42). Thus

$$
\int_{v(\alpha)=0} W_{\varphi}^{-}\left(\left(\begin{array}{ll}
\alpha & 0 \\
0 & 1
\end{array}\right)\left(\begin{array}{cc}
1 & 0 \\
\varpi^{c-1} & 1
\end{array}\right)\right) d^{*} \alpha=-\frac{1}{q-1} .
$$


Part (2) is proved.

Now to integrate $W_{\varphi}^{-}\left(\left(\begin{array}{ll}\alpha & 0 \\ 0 & 1\end{array}\right)\left(\begin{array}{cc}1 & 0 \\ \varpi^{i} & 1\end{array}\right)\right)$ against $\psi\left(\varpi^{-i} \alpha\right)$, the idea is to interpret $\psi\left(\varpi^{-i} \alpha\right)$ as a factor one can get by the group action in the Kirillov model. More specifically,

$$
\begin{aligned}
W_{\varphi}^{-}\left(\left(\begin{array}{ll}
\alpha & 0 \\
0 & 1
\end{array}\right)\left(\begin{array}{cc}
1 & 0 \\
\varpi^{i} & 1
\end{array}\right)\right) \psi\left(\varpi^{-i} \alpha\right) & =\hat{\pi}\left(\left(\begin{array}{cc}
1 & 0 \\
\varpi^{i} & 1
\end{array}\right)\right) \varphi(\alpha) \psi\left(\varpi^{-i} \alpha\right) \\
& =\hat{\pi}\left(\left(\begin{array}{cc}
1 & -\varpi^{-i} \\
0 & 1
\end{array}\right)\left(\begin{array}{cc}
1 & 0 \\
\varpi^{i} & 1
\end{array}\right)\right) \varphi(\alpha) \\
& =\hat{\pi}\left(-\omega\left(\begin{array}{cc}
\varpi^{i} & 1 \\
0 & \varpi^{-i}
\end{array}\right)\right) \varphi(\alpha) .
\end{aligned}
$$

The integral of $W_{\varphi}^{-}\left(\left(\begin{array}{ll}\alpha & 0 \\ 0 & 1\end{array}\right)\left(\begin{array}{cc}1 & 0 \\ \varpi^{i} & 1\end{array}\right)\right) \psi\left(\varpi^{-i} \alpha\right)$ is then the same as to find level 0 component of the expression above. One can do this similarly as in the proof of part (2). We will leave the rest to the reader.

Now we will give the formula for $\mathbb{P}\left(s, w, f, \Phi_{s}\right)$. We will basically follow the technique used for the unramified special representation case in the last subsection, so we will skip some details and give results directly. Recall that we have chosen

$$
f=\operatorname{char}\left(\left(\begin{array}{cc}
1 & 0 \\
\sqrt{D} & 1
\end{array}\right)\left(\begin{array}{cc}
O_{F} & O_{F} \\
\varpi^{c} O_{F} & O_{F}
\end{array}\right)\right) \times \operatorname{char}\left(O_{F}^{*}\right)
$$

It is $K_{1}\left(\varpi^{c}\right)$-invariant under the right action and the Weil representation. One can calculate for $0 \leq i \leq c$ that

$$
\begin{aligned}
r^{\prime}\left(\left(\begin{array}{cc}
1 & 0 \\
\varpi^{i} & 1
\end{array}\right)\right) f= & q^{2(i-c)} \sum_{a_{0} \in O_{F} / \varpi^{c} O_{F}} \psi\left(u \varpi^{-i}\left[\left(x_{1}-a_{0}\right) x_{4}-x_{2}\left(x_{3}-a_{0} \sqrt{D}\right)\right]\right) \\
& \times \operatorname{char}\left(\left(\begin{array}{cc}
a_{0}+\varpi^{i} O_{F} & \varpi^{i-c} O_{F} \\
a_{0} \sqrt{D}+\varpi^{i} O_{F} & \varpi^{i-c} O_{F}
\end{array}\right)\right) \times \operatorname{char}\left(O_{F}^{*}\right) .
\end{aligned}
$$

The sum is right $K_{1}\left(\varpi^{c}\right)$-invariant for any $i$.

The local integral can be written as

$$
\begin{aligned}
\mathbb{P}\left(s, w, f, \Phi_{s}\right)= & \sum_{0 \leq i \leq c} A_{i} \int_{v(\alpha)=\min \{0,2 i-c\}} W_{\varphi}^{-}\left(\left(\begin{array}{cc}
\alpha & 0 \\
0 & 1
\end{array}\right)\left(\begin{array}{cc}
1 & 0 \\
\varpi^{i} & 1
\end{array}\right)\right)|\alpha|^{\frac{w}{2}-\frac{1}{4}} \Phi_{s}(\alpha)^{-1} \\
& \times I\left(\alpha, r^{\prime}\left(\left(\begin{array}{cc}
1 & 0 \\
\varpi^{i} & 1
\end{array}\right)\right) f, \Phi_{s}\right) d^{*} \alpha
\end{aligned}
$$

where $A_{i}$ 's were given in Lemma 2.2.

$$
\text { Recall that } \Phi_{s}=\Phi_{s}^{(1)} \Phi_{s}^{(2)} \text {, and } \gamma_{0} \text { should be understood as }\left(\begin{array}{cc}
1 & 0 \\
(\sqrt{D},-\sqrt{D}) & 1
\end{array}\right) \cdot \Phi_{s}^{(2)} \text { is }
$$
the unique right $K_{1}\left(\varpi^{c}\right)$-invariant function supported on $B K_{1}\left(\varpi^{c}\right)$ such that $\Phi_{s}^{(2)}(1)=1$, and $\Phi_{s}^{(1)}$ is the standard right $K$-invariant function. Then 


$$
\begin{aligned}
& I\left(\alpha, r^{\prime}\left(\left(\begin{array}{cc}
1 & 0 \\
\varpi^{i} & 1
\end{array}\right)\right) f, \Phi_{s}\right) \\
& \quad=A_{c} \int \Phi_{s}^{(1)}\left(\left(\begin{array}{cc}
1 & 0 \\
2 \sqrt{D} & 1
\end{array}\right)\left(\begin{array}{cc}
a_{1} & m \\
0 & a_{2}
\end{array}\right)\right) \Phi_{s}^{(2)}\left(\left(\begin{array}{cc}
a_{1} & m \\
0 & a_{2}
\end{array}\right)\right) \\
& \quad \times r^{\prime}\left(\left(\begin{array}{cc}
1 & 0 \\
\varpi^{i} & 1
\end{array}\right)\right) f\left(\left(\begin{array}{cc}
a_{1} & m \\
a_{1} \sqrt{D} & a_{2}+m \sqrt{D}
\end{array}\right), \frac{\alpha}{a_{1} a_{2}}\right) d m d^{*} a_{2}\left|a_{1}\right|^{-1} d^{*} a_{1},
\end{aligned}
$$

where

$$
\begin{aligned}
& r^{\prime}\left(\left(\begin{array}{cc}
1 & 0 \\
\varpi^{i} & 1
\end{array}\right)\right) f\left(\left(\begin{array}{cc}
a_{1} & m \\
a_{1} \sqrt{D} & a_{2}+m \sqrt{D}
\end{array}\right), \frac{\alpha}{a_{1} a_{2}}\right) \\
& =q^{2(i-c)} \sum_{a_{0} \in O_{F} / \varpi^{c} O_{F}} \psi\left(\varpi^{-i} \alpha\left(1-\frac{a_{0}}{a_{1}}\right)\right) \operatorname{char}\left(\left(\begin{array}{cc}
a_{0}+\varpi^{i} O_{F} & \varpi^{i-c} O_{F} \\
a_{0} \sqrt{D}+\varpi^{i} O_{F} & \varpi^{i-c} O_{F}
\end{array}\right)\right) \\
& \quad \times\left(\left(\begin{array}{cc}
a_{1} & m \\
a_{1} \sqrt{D} & a_{2}+m \sqrt{D}
\end{array}\right)\right) .
\end{aligned}
$$

For each $a_{0}$, the corresponding term in the above expression is not zero if and only if

$$
a_{1} \equiv a_{0} \quad \bmod \left(\varpi^{i} O_{F}\right), m, a_{2} \in \varpi^{i-c} O_{F} .
$$

Lemma 5.11 For any fixed $0 \leq i \leq c$ and fixed $v(\alpha), I\left(\alpha, r^{\prime}\left(\left(\begin{array}{cc}1 & 0 \\ \varpi^{i} & 1\end{array}\right)\right) f, \Phi_{s}\right)$ as a function of $\alpha$ is a linear combination of the constant function independent of $\alpha$ and $\psi\left(\varpi^{-i} \alpha\right)$.

Proof For fixed $a_{0}$, the corresponding term in $I\left(\alpha, r^{\prime}\left(\left(\begin{array}{cc}1 & 0 \\ \varpi^{i} & 1\end{array}\right)\right) f, \Phi_{s}\right)$ is

$$
\begin{aligned}
& A_{c} q^{2(i-c)} \int_{\substack{a_{1} \equiv a_{0} \bmod \left(\varpi^{i}\right) \\
v\left(a_{1} a_{2}\right)=v(\alpha) \\
v\left(a_{2}\right), v(m) \geq i-c}} \Phi_{s}^{(1)}\left(\left(\begin{array}{cc}
a_{1} & m \\
2 a_{1} \sqrt{D} & a_{2}+2 m \sqrt{D}
\end{array}\right)\right) \chi_{1, s}^{(2)}\left(a_{1}\right) \chi_{2, s}^{(2)}\left(a_{2}\right) \\
& \times \psi\left(\varpi^{-i} \alpha\left(1-\frac{a_{0}}{a_{1}}\right)\right) d m d^{*} a_{2}\left|a_{1}\right|^{-1} d^{*} a_{1} .
\end{aligned}
$$

There are two cases. If $a_{0} \in \varpi^{i} O_{F}$, the domain for $a_{1}$ is $\varpi^{i} O_{F}$. We fix $a_{2}$ and $m$ and integrate in $a_{1}$ for fixed $v\left(a_{1}\right)$ first. Note that $\Phi_{s}^{(1)}\left(\left(\begin{array}{cc}a_{1} & m \\ 2 a_{1} \sqrt{D} & a_{2}+2 m \sqrt{D}\end{array}\right)\right)$ only depends on $v\left(a_{1}\right)$ instead of the specific value of $a_{1}$, as one can see from Lemma 4.2. So we are essentially integrating

$$
\psi\left(\varpi^{-i} \alpha\left(1-\frac{a_{0}}{a_{1}}\right)\right)=\psi\left(\varpi^{-i} \alpha\right) \psi\left(-\varpi^{-i} \alpha \frac{a_{0}}{a_{1}}\right) .
$$

Then we get either 0 or a multiple of $\psi\left(\varpi^{-i} \alpha\right)$.

If $a_{0} \notin \varpi^{i} O_{F}$, we consider the sum in $a_{0}$ for fixed $v\left(a_{0}\right)<i$. Note that $v\left(a_{1}\right)=v\left(a_{0}\right)$ would also be fixed. As the value of $\Phi_{s}$ and the domains for the integrals in $m$ and $a_{2}$ 
are actually independent of $a_{0}$, we can change the order of the integral in $a_{2}, m$ and the summation in $a_{0}$. Then the sum in $a_{0}$ is essentially

$$
\begin{aligned}
& \sum_{a_{0}} \int_{a_{1} \equiv a_{0}} \psi\left(\varpi^{-i} \alpha \frac{a_{1}-a_{0}}{a_{1}}\right) d^{*} a_{1} \\
& =\int_{a_{1}} \sum_{a_{0} \equiv a_{1}} \sum_{\bmod \left(\varpi^{i}\right)} \psi\left(\varpi^{-i} \alpha \frac{a_{1}-a_{0}}{a_{1}}\right) d^{*} a_{1} .
\end{aligned}
$$

One can now easily see that the inner sum is either 0 or a constant independent of $\alpha$.

As a result of this Lemma and Lemma 5.10, we only have to care about the constant part when $i=c, c-1$ and the $\psi\left(\varpi^{-i} \alpha\right)$ part when $i=0,1$.

Lemma 5.12 Suppose that $v(\alpha)=\min \{0,2 i-c\}$.

(1) $I\left(\alpha, f, \Phi_{s}\right)=A_{c}$.

The constant part of $I\left(\alpha, r^{\prime}\left(\left(\begin{array}{cc}1 & 0 \\ \varpi^{c-1} & 1\end{array}\right)\right) f\right.$, $\left.\Phi_{s}\right)$ is $A_{c}\left[q^{-1}+\left(1-q^{-1}\right) \frac{\chi_{1, s}^{(1)}}{\chi_{2, s}^{(1)}}\right]$.

(2) $I\left(\alpha, r^{\prime}\left(\left(\begin{array}{ll}1 & 0 \\ 1 & 1\end{array}\right)\right) f, \Phi_{s}\right)=0$.

The $\psi\left(\varpi^{-1} \alpha\right)$ part of I $\left(\alpha, r^{\prime}\left(\left(\begin{array}{cc}1 & 0 \\ \varpi & 1\end{array}\right)\right) f, \Phi_{s}\right)$ is 0.

We will however not provide proof here. Basically one can use (5.50) to do the calculations, and at some steps switch the order of the integral and the summation in $a_{0}$ as in the proof of the previous lemma. It's complicated, but not difficult.

Now we combine Lemmas 5.10 and 5.12 to compute (5.46). Note that only $i=0,1$ terms are non-zero, and $v(\alpha)=0$ for these terms. Then

$$
\begin{aligned}
\mathbb{P}\left(s, w, f, \Phi_{s}\right)= & A_{c} \int_{\nu(\alpha)=0} W_{\varphi}^{-}\left(\left(\begin{array}{ll}
\alpha & 0 \\
0 & 1
\end{array}\right)\right) I\left(\alpha, f, \Phi_{s}\right) d^{*} \alpha \\
& +A_{c-1} \int_{v(\alpha)=0} W_{\varphi}^{-}\left(\left(\begin{array}{ll}
\alpha & 0 \\
0 & 1
\end{array}\right)\left(\begin{array}{cc}
1 & 0 \\
\varpi^{c-1} & 1
\end{array}\right)\right) \\
& \times I\left(\alpha, r^{\prime}\left(\left(\begin{array}{cc}
1 & 0 \\
\varpi^{c-1} & 1
\end{array}\right)\right) f, \Phi_{s}\right) d^{*} \alpha \\
= & A_{c} \cdot A_{c}+A_{c-1}\left(-\frac{1}{q-1}\right) A_{c}\left[q^{-1}+\left(1-q^{-1}\right) \frac{\chi_{1, s}^{(1)}}{\chi_{2, s}^{(1)}}\right] \\
= & \frac{1-q^{-1}}{(q+1)^{2} q^{2 c-2}}\left(1-\frac{\chi_{1}^{(1)}}{\chi_{2}^{(1)}} q^{-(2 s+1)}\right) .
\end{aligned}
$$

Note that this result is independent of $w$.

\subsubsection{Highly ramified principal series}

Now we consider the case when $\pi \simeq \pi\left(\mu_{1}, \mu_{2}\right)$ is highly ramified. In the case when $\pi$ is a highly ramified special representation, we will get the same result as we can choose the same new form. 
We still assume that $\chi_{1}$ and $\chi_{2}$ are unramified and $\mathbb{E} / \mathbb{F}$ is split. This implies that $\mu_{1}$ and $\mu_{2}$ should be ramified of the same level $k$. Let $c=2 k \geq 2$ be the level of $\pi$ and $\hat{\pi}$. It's a classical result (refer to [5]) that the $K_{1}\left(\varpi^{c}\right)$-invariant new form $\varphi$ is supported on $B\left(\begin{array}{cc}1 & 0 \\ \varpi^{k} & 1\end{array}\right) K_{1}\left(\varpi^{c}\right)$.

The idea is to compare the Whittaker function for $\varphi$ with Lemma 5.10. If we can get similar properties, then we can choose the same $f$ and $\Phi_{s}$ as in the previous situation and get the results directly.

Lemma 5.13 (1) If $k<i \leq c$, then $W_{\varphi}^{-}\left(\left(\begin{array}{cc}\alpha & 0 \\ 0 & 1\end{array}\right)\left(\begin{array}{cc}1 & 0 \\ \varpi^{i} & 1\end{array}\right)\right)$ is not zero only when $v(\alpha)=0$. In that case, its integral against $\psi\left(\varpi^{-i} \alpha\right)$ is always 0 .

$$
\int_{\nu(\alpha)=0} W_{\varphi}^{-}\left(\left(\begin{array}{cc}
\alpha & 0 \\
0 & 1
\end{array}\right)\left(\begin{array}{cc}
1 & 0 \\
\varpi^{i} & 1
\end{array}\right)\right) d^{*} \alpha= \begin{cases}1, & \text { if } i=c ; \\
-\frac{1}{q-1}, & \text { if } i=c-1>k \\
0, & \text { otherwise } .\end{cases}
$$

(2) If $i<k$, then $W_{\varphi}^{-}\left(\left(\begin{array}{ll}\alpha & 0 \\ 0 & 1\end{array}\right)\left(\begin{array}{cc}1 & 0 \\ \varpi^{i} & 1\end{array}\right)\right)$ is not zero only when $v(\alpha)=2 i-c$. In that case, its integral against 1 is always 0.

$$
\begin{aligned}
& \int_{v(\alpha)=2 i-c} W_{\varphi}^{-}\left(\left(\begin{array}{cc}
\alpha & 0 \\
0 & 1
\end{array}\right)\left(\begin{array}{cc}
1 & 0 \\
\varpi^{i} & 1
\end{array}\right)\right) \psi\left(\varpi^{-i} \alpha\right) d^{*} \alpha \\
& =w_{\pi}\left(\varpi^{k-i}\right) \mu_{1}^{-1}(-1) \begin{cases}1, & \text { if } i=0 ; \\
-\frac{1}{q-1}, & \text { if } i=1<k ; \\
0, & \text { otherwise. }\end{cases}
\end{aligned}
$$

(3) If $i=k$, the integral of $W_{\varphi}^{-}\left(\left(\begin{array}{ll}\alpha & 0 \\ 0 & 1\end{array}\right)\left(\begin{array}{cc}1 & 0 \\ \varpi^{k} & 1\end{array}\right)\right)$ against 1 or $\psi\left(\varpi^{-k} \alpha\right)$ is always zero if either $k>1$ or $v(\alpha) \neq 0$. When $k=1$ and $v(\alpha)=0$, its integral against 1 is the same as expected from (1) as the limit case, and its integral against $\psi\left(\varpi^{-k} \alpha\right)$ is the same as expected from (2).

Proof According to part (3) of Lemma 5.6, we can always write

$$
\begin{aligned}
W_{\varphi}^{-}\left(\left(\begin{array}{ll}
\alpha & 0 \\
0 & 1
\end{array}\right)\left(\begin{array}{cc}
1 & 0 \\
\varpi^{i} & 1
\end{array}\right)\right)= & \int \mu_{1}^{-1}\left(-\frac{\alpha \varpi^{k}}{\alpha+m \varpi^{i}}\right) \mu_{2}^{-1}(-m) \\
& \times\left|\frac{\alpha \varpi^{k}}{m\left(\alpha+m \varpi^{i}\right)}\right|^{1 / 2} \psi(m) d m .
\end{aligned}
$$

The difference is the domain for $m$, which was given in Lemma 5.6. For the sake of conciseness, we will only prove part (1) here.

When $k<i \leq c$, the domain for $m$ is $v(m)=v(\alpha)-k$. Write $m=\varpi^{-k} \alpha u$ for $u \in O_{F}^{*}$. The integral becomes

$$
\begin{gathered}
W_{\varphi}^{-}\left(\left(\begin{array}{cc}
\alpha & 0 \\
0 & 1
\end{array}\right)\left(\begin{array}{cc}
1 & 0 \\
\varpi^{i} & 1
\end{array}\right)\right)=\int_{u \in O_{F}^{*}} \mu_{1}^{-1}\left(-\frac{\varpi^{k}}{1+u \varpi^{i-k}}\right) \mu_{2}^{-1}\left(-\varpi^{-k} \alpha u\right) \\
\times q^{-v(\alpha) / 2} \psi\left(\varpi^{-k} \alpha u\right) d u .
\end{gathered}
$$


As functions in $u, \mu_{1}^{-1}\left(-\frac{\varpi^{k}}{1+u \varpi^{i-k}}\right)$ is at most of level $2 k-i<k, \mu_{2}^{-1}\left(-\varpi^{-k} \alpha u\right)$ is multiplicative of level $k$ and $\psi\left(-\varpi^{-k} \alpha u\right)$ is additive of level $k-v(\alpha)$. So if $v(\alpha) \neq 0$, the integral will be zero for level reason.

When $v(\alpha)=0$ and $i=c, \mu_{1}^{-1}\left(-\frac{\varpi^{k}}{1+u \varpi^{i-k}}\right)=\mu_{1}^{-1}\left(-\varpi^{k}\right)$ as $\mu_{1}$ is level $k$. Then

$$
\begin{aligned}
W_{\varphi}^{-}\left(\left(\begin{array}{ll}
1 & 0 \\
0 & 1
\end{array}\right)\right) & =\mu_{1}^{-1}\left(-\varpi^{k}\right) \int_{u \in O_{F}^{*}} \mu_{2}^{-1}\left(-\varpi^{-k} \alpha u\right) \psi\left(\varpi^{-k} \alpha u\right) d u \\
& =\mu_{1}^{-1}\left(\varpi^{k}\right) \int_{u \in O_{F}^{*}} \mu_{2}^{-1}\left(\varpi^{-k} u\right) \psi\left(\varpi^{-k} u\right) d^{*} u \cdot\left(1-q^{-1}\right) .
\end{aligned}
$$

We have used $\mu_{1} \mu_{2}(-1)=1$ here.

We first consider the integral

$$
\int_{v(\alpha)=0} W_{\varphi}^{-}\left(\left(\begin{array}{ll}
\alpha & 0 \\
0 & 1
\end{array}\right)\left(\begin{array}{cc}
1 & 0 \\
\varpi^{i} & 1
\end{array}\right)\right) d^{*} \alpha
$$

One can switch the order and integrate in $\alpha$ first. Note that

$$
\left.\left.\int_{v(\alpha)} \psi\left(\varpi^{-k} \alpha u\right)\right) \mu_{2}^{-1}\left(-\varpi^{-k} \alpha u\right) d^{*} \alpha=\int_{\nu(\alpha)} \psi\left(\varpi^{-k} \alpha\right)\right) \mu_{2}^{-1}\left(-\varpi^{-k} \alpha\right) d^{*} \alpha
$$

is independent of $u \cdot q^{-v(\alpha)}=1$. So

$$
\begin{aligned}
& \int_{v(\alpha)=0} W_{\varphi}^{-}\left(\left(\begin{array}{ll}
\alpha & 0 \\
0 & 1
\end{array}\right)\left(\begin{array}{cc}
1 & 0 \\
\varpi^{i} & 1
\end{array}\right)\right) d^{*} \alpha \\
& \left.=\int_{u \in O_{F}^{*}} \mu_{1}^{-1}\left(-\frac{\varpi^{k}}{1+u \varpi^{i-k}}\right) d u \int_{\nu(\alpha)} \psi\left(\varpi^{-k} \alpha\right)\right) \mu_{2}^{-1}\left(-\varpi^{-k} \alpha\right) d^{*} \alpha \\
& \left.=\mu_{1}^{-1}\left(\varpi^{k}\right) \int_{\nu(\alpha)} \psi\left(\varpi^{-k} \alpha\right)\right) \mu_{2}^{-1}\left(\varpi^{-k} \alpha\right) d^{*} \alpha \cdot \begin{cases}1-q^{-1}, & \text { if } i=c ; \\
-q^{-1}, & \text { if } i=c-1>k ; \\
0, & \text { otherwise. }\end{cases}
\end{aligned}
$$

In the last equation we have used Lemma 2.4.

Now we integrate $W_{\varphi}^{-}\left(\left(\begin{array}{cc}\alpha & 0 \\ 0 & 1\end{array}\right)\left(\begin{array}{cc}1 & 0 \\ \varpi^{i} & 1\end{array}\right)\right)$ against $\psi\left(\varpi^{-i} \alpha\right)$ when $v(\alpha)=0$ and $k<i \leq c$. Again we shall switch the order of the integrals and get

$$
\begin{aligned}
& \int_{\nu(\alpha)=0} W_{\varphi}^{-}\left(\left(\begin{array}{ll}
\alpha & 0 \\
0 & 1
\end{array}\right)\left(\begin{array}{cc}
1 & 0 \\
\varpi^{i} & 1
\end{array}\right)\right) \psi\left(\varpi^{-i} \alpha\right) d^{*} \alpha \\
& =\int_{u \in O_{F}^{*}} \mu_{1}^{-1}\left(-\frac{\varpi^{k}}{1+u \varpi^{i-k}}\right) \int_{v(\alpha)=0} \mu_{2}^{-1}\left(-\varpi^{-k} \alpha u\right) \psi\left(\varpi^{-k} \alpha u\right) \psi\left(\varpi^{-i} \alpha\right) d^{*} \alpha d u .
\end{aligned}
$$

As functions in $\alpha, \mu_{2}^{-1}\left(-\varpi^{-k} \alpha u\right)$ is of level $k$, and $\psi\left(\varpi^{-i} \alpha\left(1+u \varpi^{i-k}\right)\right)$ is of level $i>k$. Thus the integral in $\alpha$ would be zero.

Lastly when we normalize $W_{\varphi}^{-}\left(\left(\begin{array}{ll}1 & 0 \\ 0 & 1\end{array}\right)\right)$ to be 1 , we will get the formulae as claimed. 


\subsubsection{Normalization}

If we use $f^{\prime}$ to do Theta lifting, we will get Gross-Prasad test vectors for both $F_{1}$ and $F$. Let

$$
f^{\prime \prime}=\operatorname{char}\left(\left(\begin{array}{cc}
O_{F} & O_{F} \\
\varpi^{c} O_{F} & O_{F}
\end{array}\right)\right) \times \operatorname{char}\left(O_{F}^{*}\right)
$$

We can compute $\left\langle F_{1}, F\right\rangle$ using $f^{\prime \prime}$ just as in (5.31):

$$
\begin{aligned}
\left\langle F_{1}, F\right\rangle= & \sum_{0 \leq i \leq c} A_{i} \iint W_{\varphi}^{-}\left(\left(\begin{array}{ll}
\alpha & 0 \\
0 & 1
\end{array}\right)\left(\begin{array}{cc}
1 & 0 \\
\varpi^{i} & 1
\end{array}\right)\right) r^{\prime}\left(\left(\begin{array}{cc}
1 & 0 \\
\varpi^{i} & 1
\end{array}\right)\right) \\
& \times f^{\prime \prime}\left(\alpha x, \alpha^{-1} x^{-2}\right) w_{\hat{\pi}}(x) d^{*} x d^{*} \alpha
\end{aligned}
$$

Only $i=c$ or $c-1$ has non-zero contribution because of Lemmas 5.10, 5.13 and the fact that $f^{\prime \prime}$ is invariant under the action of center. Then one can easily compute that

$$
\left\langle F_{1}, F\right\rangle=A_{c}+A_{c-1}\left(-\frac{1}{q-1}\right) q^{-1}=\frac{q-1}{q+1} \frac{1}{q^{c}} .
$$

The expected L-factors in this case is

$$
\begin{aligned}
& \frac{\zeta(2)}{L(\pi, A d, 1)} \frac{L(\Pi \otimes \Omega, 1 / 2) L\left(\left.\pi \otimes \chi_{1}\right|_{\left.\mathbb{F}^{*}, 2 s+1 / 2\right)}\right.}{L(\eta, 1) L(\chi, 2 s+1)} \\
& \quad=\frac{1}{\left(1-q^{-2}\right) L(\pi, A d, 1)}\left(1-q^{-1}\right)\left(1-\chi^{(1)} q^{-(2 s+1)}\right)\left(1-\chi^{(2)} q^{-(2 s+1)}\right) .
\end{aligned}
$$

Then

$$
\mathbb{P}^{0}\left(s, 1 / 2, f^{\prime}, \Phi_{s}^{\prime}\right)=\frac{1}{q^{c}} \frac{L(\pi, A d, 1)}{1-\chi^{(2)} q^{-(2 s+1)}} .
$$

\subsection{Ramification in $\Phi_{s}$}

In this subsection, we consider the case when $\Phi_{s}$ is ramified while $\pi$ is unramified. To make things simple, we assume that $\chi_{1}$ is ramified of level $c$ and $\chi_{2}$ is unramified. Note $\left.\mu_{1} \mu_{2}\left(\chi_{1} \chi_{2}\right)\right|_{\mathbb{F}^{*}}=1$ implies that $\left.\chi_{1}\right|_{\mathbb{F}^{*}}$ is still unramified.

Proposition 5.14 Suppose that $\pi$ is unramified and $\mathbb{E} / \mathbb{F}$ is inert. Suppose that $\chi_{1}$ is ramified of level c, but $\left.\chi_{1}\right|_{\mathbb{F}^{*}}$ and $\chi_{2}$ are unramified. Pick

$$
f=\operatorname{char}\left(\left(\begin{array}{cc}
O_{F} & \varpi^{c} O_{F} \\
\varpi^{-c} O_{F} & O_{F}
\end{array}\right)\right) \times \operatorname{char}\left(O_{F}^{*}\right) .
$$

Pick $\Phi_{s}$ to be the new form, that is, the $K_{1}\left(\varpi^{c}\right)$-invariant function supported on $B\left(\begin{array}{ll}1 & 0 \\ 1 & 1\end{array}\right) K_{1}\left(\varpi^{c}\right)$. Then

$$
\mathbb{P}\left(s, w, f, \Phi_{s}\right)=\frac{\mathbb{P}^{\prime}(w)}{\left(1-\delta \mu_{1} \chi_{1, s}(\varpi) q\right)\left(1-\delta \mu_{2} \chi_{1, s}(\varpi) q\right)},
$$

where $\mathbb{P}^{\prime}(w)$ denotes the expression

$$
\begin{aligned}
& \frac{\left(\frac{\chi_{2, s}}{q \chi_{1, s}}\right)^{-c}}{\mu_{2}-\mu_{1}}\left[\mu_{2} \frac{\left(1-\left(\delta \mu_{2} \chi_{2, s}\right)^{c+1}\right)-\frac{\chi_{2, s}}{q^{2} \chi_{1, s}}\left(1-\left(\delta \mu_{2} \chi_{2, s}\right)^{c}\right)}{1-\delta \mu_{2} \chi_{2, s}}\left(1-q \delta \mu_{1} \chi_{1, s}\right)\right. \\
& \left.-\mu_{1} \frac{\left(1-\left(\delta \mu_{1} \chi_{2, s}\right)^{c+1}\right)-\frac{\chi_{2, s}}{q^{2} \chi_{1, s}}\left(1-\left(\delta \mu_{1} \chi_{2, s}\right)^{c}\right)}{1-\delta \mu_{1} \chi_{2, s}}\left(1-q \delta \mu_{2} \chi_{1, s}\right)\right] .
\end{aligned}
$$


Here $\delta=q^{-(w / 2+1 / 4)}$. When $w=1 / 2$,

$$
\mathbb{P}^{0}\left(s, 1 / 2, f, \Phi_{s}\right)=\frac{\mathbb{P}^{\prime}(1 / 2)}{1+q^{-1}}
$$

Recall the local integral

$$
\mathbb{P}\left(s, w, f, \Phi_{s}\right)=\int_{Z N \backslash \mathrm{GL}_{2}(\mathbb{F})} W_{\varphi}^{-}(h) \Delta(h)^{w-1 / 2} \int_{\mathrm{GL}_{2}(\mathbb{F})} r^{\prime}(h) f\left(g, \operatorname{det}(g)^{-1}\right) \Phi_{s}\left(\gamma_{0} g\right) d g d h .
$$

We've already known well the $K$-invariant Whittaker function $W_{\varphi}^{-}$. The choice

$$
f=\operatorname{char}\left(\left(\begin{array}{cc}
O_{F} & \varpi^{c} O_{F} \\
\varpi^{-c} O_{F} & O_{F}
\end{array}\right)\right) \times \operatorname{char}\left(O_{F}^{*}\right)
$$

is motivated by Example 2.21. This Schwartz function is $K$-invariant under the Weil representation. Thus as in the unramified case,

$$
\mathbb{P}\left(s, w, f, \Phi_{s}\right)=\int_{\mathbb{F}^{*}} W_{\varphi}^{-}\left(\left(\begin{array}{ll}
\alpha & 0 \\
0 & 1
\end{array}\right)\right)|\alpha|^{w / 2-1 / 4} \Phi_{s}(\alpha)^{-1} I\left(\alpha, f, \Phi_{s}\right) d^{*} \alpha .
$$

The key point in this subsection is to work out

$$
I\left(\alpha, f, \Phi_{s}\right)=\int_{g \in \mathrm{GL}_{2}, v(\operatorname{det} g)=v(\alpha)} f\left(g, \frac{\alpha}{\operatorname{det} g}\right) \Phi_{s}\left(\gamma_{0} g\right) d g .
$$

Note that when the extension $\mathbb{E} / \mathbb{F}$ is inert,

$$
\mathrm{GL}_{2}=O_{E}^{*} \cdot B \text {. }
$$

Recall that $\Phi_{s}\left(\gamma_{0} t g\right)=\chi_{1, s}(\bar{t}) \chi_{2, s}(t) \Phi_{s}\left(\gamma_{0} g\right)$ for $t=a+b \sqrt{D}$. By our assumption on $\chi_{1, s}$ and $\chi_{2, s}, \Phi_{s}\left(\gamma_{0} g\right)$ as a function of $g$ is left invariant under $O_{F}^{*}+\varpi^{c} O_{E} . f$ is also left invariant under $\left\{\left(\begin{array}{cc}a & b \\ b D & a\end{array}\right) \mid a \in O_{F}^{*}, b \in \varpi^{c} O_{F}\right\} \simeq O_{F}^{*}+\varpi^{c} O_{E}$. As a result,

$$
\begin{aligned}
I\left(\alpha, f, \Phi_{s}\right)= & \frac{1}{(q+1) q^{c-1}} \sum_{t \in\left(O_{F}^{*}+\varpi^{c} O_{E}\right) \backslash O_{E}^{*}} \int_{B} \chi_{1, s}(\bar{t}) \Phi_{s}\left(\gamma_{0} b\right) f\left(t b, \frac{\alpha}{\operatorname{det} t b}\right) d b \\
= & \frac{1}{(q+1) q^{c-1}} \sum_{t \in\left(O_{F}^{*}+\varpi^{c} O_{E}\right) \backslash O_{E}^{*}} \int_{t\left(\begin{array}{cc}
a_{1} & m \\
0 & a_{2}
\end{array}\right) \in\left(\begin{array}{cc}
O_{F} & \varpi^{c} O_{F} \\
\varpi^{-c} O_{F} & O_{F}
\end{array}\right)} \\
& \times \Phi_{s}\left(\gamma_{0}\left(\begin{array}{cc}
a_{1} & m \\
0 & a_{2}
\end{array}\right)\right)\left|a_{2}\right|^{-1} d m d^{*} a_{1} d^{*} a_{2} .
\end{aligned}
$$

We have used that the right Haar measure for the Borel subgroup is

$$
\left|a_{2}\right|^{-1} d m d^{*} a_{1} d^{*} a_{2} .
$$

The coset representatives $\left(O_{F}^{*}+\varpi^{c} O_{E}\right) \backslash O_{E}^{*}$ can be chosen as

$$
\left\{1+b_{1} \sqrt{D} \mid b_{1} \in O_{F} / \varpi^{c} O_{F}\right\} \cup\left\{b_{2}+\sqrt{D} \mid b_{2} \in \varpi O_{F} / \varpi^{c} O_{F}\right\} .
$$

One can easily see that this set has $(q+1) q^{c-1}$ elements. That's why we have $\frac{1}{(q+1) q^{c-1}}$ in front of the integral above. 
Lemma 5.15 We need $v\left(\frac{a_{1} \sqrt{D}}{a_{2}+m \sqrt{D}}\right) \leq 0$ for

$$
\left(\begin{array}{cc}
a_{1} & m \\
a_{1} \sqrt{D} & a_{2}+m \sqrt{D}
\end{array}\right) \in B\left(\begin{array}{ll}
1 & 0 \\
1 & 1
\end{array}\right) K_{1}\left(\varpi^{c}\right) .
$$

Under the above condition we can write $\left(\begin{array}{cc}a_{1} & m \\ a_{1} \sqrt{D} & a_{2}+m \sqrt{D}\end{array}\right)$ as

$$
\left(\begin{array}{cc}
\frac{a_{2}}{\sqrt{D}} & a_{1}-\frac{a_{2}}{\sqrt{D}} \\
0 & a_{1} \sqrt{D}
\end{array}\right)\left(\begin{array}{ll}
1 & 0 \\
1 & 1
\end{array}\right)\left(\begin{array}{cc}
1 & -1+\frac{a_{2}+m \sqrt{D}}{a_{1} \sqrt{D}} \\
0 & 1
\end{array}\right)
$$

Corollary 5.16 Assume that $\chi_{2, s}$ is unramified and $\chi_{1, s}$ is ramified of level c, such that $\left.\chi_{1, s}\right|_{\mathbb{F}^{*}}$ is still unramified. Suppose that $\Phi_{s}$ is the unique $K_{1}\left(\varpi^{c}\right)$-invariant function supported on $B\left(\begin{array}{ll}1 & 0 \\ 1 & 1\end{array}\right) K_{1}\left(\varpi^{c}\right)$. Then $\Phi_{s}\left(\left(\begin{array}{cc}1 & 0 \\ \sqrt{D} & 1\end{array}\right)\left(\begin{array}{cc}a_{1} & m \\ 0 & a_{2}\end{array}\right)\right)$ is non-zero when $v\left(a_{1}\right) \leq v\left(a_{2}+m \sqrt{D}\right)$. In that case, it's equal to

$$
\chi_{1, s}\left(\frac{a_{2}}{\sqrt{D}}\right) \chi_{2, s}\left(a_{1} \sqrt{D}\right)=\frac{\chi_{1, s}^{\nu\left(a_{2}\right)} \chi_{2, s}^{\nu\left(a_{1}\right)}}{\chi_{1, s}(\sqrt{D})} .
$$

For each representative $t \in\left(O_{F}^{*}+\varpi^{c} O_{E}\right) \backslash O_{E}^{*}$, we decide now the domain of the integral which is given by the condition

$$
t\left(\begin{array}{cc}
a_{1} & m \\
0 & a_{2}
\end{array}\right) \in\left(\begin{array}{cc}
O_{F} & \varpi^{c} O_{F} \\
\varpi^{-c} O_{F} & O_{F}
\end{array}\right) .
$$

If $t=1+b_{1} \sqrt{D}$, then by

$$
\left(\begin{array}{cc}
1 & b_{1} \\
b_{1} D & 1
\end{array}\right)\left(\begin{array}{cc}
a_{1} & m \\
0 & a_{2}
\end{array}\right) \in\left(\begin{array}{cc}
O_{F} & \varpi^{c} O_{F} \\
\varpi^{-c} O_{F} & O_{F}
\end{array}\right)
$$

we get $v\left(a_{1}\right), v\left(a_{2}\right) \geq 0, m \equiv-b_{1} a_{2} \bmod \left(\varpi^{c}\right)$. Similarly for $t=\sqrt{D}+b_{2}$,

$$
\left(\begin{array}{cc}
b_{2} & 1 \\
D & b_{2}
\end{array}\right)\left(\begin{array}{cc}
a_{1} & m \\
0 & a_{2}
\end{array}\right) \in\left(\begin{array}{cc}
O_{F} & \varpi^{c} O_{F} \\
\varpi^{-c} O_{F} & O_{F}
\end{array}\right) .
$$

The domain will be $v\left(a_{1}\right) \geq-v\left(b_{2}\right), v(m) \geq 0, a_{2} \equiv-m b_{2} \bmod \left(\varpi^{c}\right)$.

The key observation here is that although the domain depends on the specific choice of $b_{1}$ or $b_{2}$, the integral of $\Phi_{s}$ over the domain only depends on $v\left(b_{1}\right)$ and $v\left(b_{2}\right)$. Indeed in Corollary 5.16, the requirement that $v\left(a_{1}\right) \leq v\left(a_{2}+m \sqrt{D}\right)$ and the value of $\Phi_{s}$ both depend only on the valuations of $a_{1}, a_{2}$ and $m$. The domains differ slightly but the different parts have the same volume.

More specifically for fixed $v\left(b_{1}\right)$ or $v\left(b_{2}\right)$, let $t_{0}$ be a fixed representative for $1+b_{1} \sqrt{D}$ or $b_{2}+\sqrt{D}$. Then we have

$$
\int_{B} \chi_{1, s}(\bar{t}) \Phi_{s}\left(\gamma_{0} b\right) f\left(t b, \frac{\alpha}{\operatorname{det} t b}\right) d b=\chi_{1, s}(\bar{t}) \int_{B} \Phi_{s}\left(\gamma_{0} b\right) f\left(t_{0} b, \frac{\alpha}{\operatorname{det} t_{0} b}\right) d b .
$$

So when we sum over $t$ for fixed $v\left(b_{1}\right)$ or $v\left(b_{2}\right)$, we are essentially just summing $\chi_{1, s}(\bar{t})$. Then we need a lemma similar to Lemma 2.4:

Lemma 5.17 Let $\chi$ be a character of level $c$ on $\mathbb{E}^{*}$ which is unramified when restricted to $\mathbb{F}^{*}$. 
(1) If $c \geq 2$, we have

$$
\begin{gathered}
\sum_{b_{1} \in O_{F} / \varpi^{c} O_{F}, v\left(b_{1}\right)=i} \chi\left(1+b_{1} \sqrt{D}\right)=\left\{\begin{array}{l}
1, \text { if } i=c ; \\
-1, \text { if } i=c-1 ; \\
0, \text { otherwise. }
\end{array}\right. \\
\sum_{b_{2} \in O_{F} / \varpi^{c} O_{F}, v\left(b_{2}\right)=i} \chi\left(\sqrt{D}+b_{2}\right)=\chi(\sqrt{D})\left\{\begin{array}{l}
1, \text { if } i=c ; \\
-1, \text { if } i=c-1 ; \\
0, \text { otherwise. }
\end{array}\right.
\end{gathered}
$$

(2) If $c=1$, we have

$$
\sum_{b_{1} \in O_{F} / \varpi O_{F}} \chi\left(1+b_{1} \sqrt{D}\right)+\chi(\sqrt{D})=0 .
$$

This lemma enable us to greatly simplify (5.73) as

$$
\begin{aligned}
I\left(\alpha, f, \Phi_{s}\right)= & \frac{1}{(q+1) q^{c-1}}\left[\left(\int_{B} \Phi_{s}\left(\gamma_{0} g\right) f\left(g, \frac{\alpha}{\operatorname{det} g}\right) d g\right.\right. \\
& \left.-\int_{B} \Phi_{s}\left(\gamma_{0} g\right) f\left(\left(\begin{array}{cc}
1 & \varpi^{c-1} \\
\varpi^{c-1} D & 1
\end{array}\right) g, \frac{\alpha}{\operatorname{det} g}\right) d g\right) \\
& +\chi_{1, s}(\sqrt{D})\left(\int_{B} \Phi_{s}\left(\gamma_{0} g\right) f\left(\begin{array}{cc}
0 & 1 \\
D & 0
\end{array}\right) g, \frac{\alpha}{\operatorname{det} g}\right) d g \\
& \left.\left.-\int_{B} \Phi_{s}\left(\gamma_{0} g\right) f\left(\left(\begin{array}{cc}
\varpi^{c-1} & 1 \\
D & \varpi^{c-1}
\end{array}\right) g, \frac{\alpha}{\operatorname{det} g}\right) d g\right)\right] .
\end{aligned}
$$

Here we have chosen $\left(\begin{array}{cc}1 & \varpi^{c-1} \\ \varpi^{c-1} D & 1\end{array}\right)$ as a representative for $1+b_{1} \sqrt{D}$ with $v\left(b_{1}\right)=$ $c-1$, and $\left(\begin{array}{cc}\varpi^{c-1} & 1 \\ D & \varpi^{c-1}\end{array}\right)$ as a representative for $\sqrt{D}+b_{2}$ with $v\left(b_{2}\right)=c-1$. This formula is true even if $c=1$.

To integrate (5.77), it's easier to compare the domains of the integrals, as the common part can be cancelled when we do subtraction. We will only give the result here:

\section{Lemma 5.18}

$$
\begin{aligned}
I\left(\alpha, f, \Phi_{s}\right)= & \frac{1}{(q+1) q^{c-1}} \\
& \times \begin{cases}\left(q \chi_{1, s}\right)^{v(\alpha)}\left(\left(\frac{\chi_{2, s}}{q \chi_{1, s}}\right)^{-c}-\left(\frac{\chi_{2, s}}{q \chi_{1, s}}\right)^{\nu(\alpha)-c+1}\right) \frac{1-\frac{\chi_{2, s}}{q^{2} \chi_{1, s}}}{1-\frac{\chi_{2}, s}{\chi_{1, s}}}, & \text { if } 0 \leq v(\alpha) \leq c-1 ; \\
\left(q \chi_{1, s}\right)^{v(\alpha)}\left(\frac{\chi_{2, s}}{q \chi_{1, s}}\right)^{-c} \frac{1-\frac{\chi_{2, s}}{q^{2} \chi_{1, s}}}{1-\frac{\chi_{1, s}}{q \chi_{1, s}}}-\left(1-q^{-1}\right)\left(q \chi_{1, s}\right)^{\nu(\alpha)} \frac{\frac{\chi_{2, s}}{q \chi_{1, s}}}{1-\frac{\chi_{2}, s}{q \chi_{1, s}}}, & \text { if } v(\alpha) \geq c .\end{cases}
\end{aligned}
$$

From this one can get 


$$
\mathbb{P}\left(s, w, f, \Phi_{s}\right)=\frac{\mathbb{P}^{\prime}(w)}{\left(1-\delta \mu_{1} \chi_{1, s}(\varpi) q\right)\left(1-\delta \mu_{2} \chi_{1, s}(\varpi) q\right)},
$$

where $\mathbb{P}^{\prime}$ denotes the expression

$$
\begin{gathered}
\frac{\left(\frac{\chi_{2, s}}{q \chi_{1, s}}\right)^{-c}}{\mu_{2}-\mu_{1}}\left[\mu_{2} \frac{\left(1-\left(\delta \mu_{2} \chi_{2, s}\right)^{c+1}\right)-\frac{\chi_{2, s}}{q^{2} \chi_{1, s}}\left(1-\left(\delta \mu_{2} \chi_{2, s}\right)^{c}\right)}{1-\delta \mu_{2} \chi_{2, s}}\left(1-q \delta \mu_{1} \chi_{1, s}\right)\right. \\
\left.-\mu_{1} \frac{\left(1-\left(\delta \mu_{1} \chi_{2, s}\right)^{c+1}\right)-\frac{\chi_{2, s}}{q^{2} \chi_{1, s}}\left(1-\left(\delta \mu_{1} \chi_{2, s}\right)^{c}\right)}{1-\delta \mu_{1} \chi_{2, s}}\left(1-q \delta \mu_{2} \chi_{1, s}\right)\right] .
\end{gathered}
$$

Now we compute $\left\langle F_{1}, F\right\rangle$. Since the Schwartz function is $K$-invariant under the Weil representation, we have

$$
\left\langle F_{1}, F\right\rangle=\iint W_{\varphi}^{-}\left(\left(\begin{array}{ll}
\alpha & 0 \\
0 & 1
\end{array}\right)\right) f\left(\alpha x, \alpha^{-1} x^{-2}\right)\left(\mu_{1} \mu_{2}\right)^{-1}(x) d^{*} x d^{*} \alpha .
$$

This turns out to be the same as in the unramified case, that is,

$$
\left\langle F_{1}, F\right\rangle=\frac{L(\pi, A d, 1)}{\zeta(2)}
$$

The expected L-factors in this case is

$$
\begin{aligned}
& \frac{\zeta(2)}{L(\pi, A d, 1)} \frac{L(\Pi \otimes \Omega, 1 / 2) L\left(\left.\pi \otimes \chi_{1}\right|_{\mathbb{F}^{*}}, 2 s+1 / 2\right)}{L(\eta, 1) L(\chi, 2 s+1)} \\
& \quad=\frac{\zeta(2)}{L(\pi, A d, 1)} \frac{1+q^{-1}}{\left(1-\mu_{1} \chi_{1}(\varpi) q^{-(2 s+1 / 2)}\right)\left(1-\mu_{2} \chi_{1}(\varpi) q^{-(2 s+1 / 2)}\right)} .
\end{aligned}
$$

Thus

$$
\mathbb{P}^{0}\left(s, 1 / 2, f, \Phi_{s}\right)=\frac{\mathbb{P}^{\prime}(1 / 2)}{1+q^{-1}} .
$$

\subsection{Joint ramification}

In this section we consider a very special case of the local integral when both $\pi\left(\mu_{1}, \mu_{2}\right)$ and $\Phi_{s}$ are ramified.

Proposition 5.19 Suppose that $\mu_{1}$ is unramified and $\mu_{2}$ is of level $c>0$ for the principal series $\pi\left(\mu_{1}, \mu_{2}\right)$. Suppose that $\chi_{2}$ is unramified and $\chi_{1},\left.\chi_{1}\right|_{\mathbb{F}^{*}}$ are both ramified of level $c$. Assume that $\mathbb{E} / \mathbb{F}$ is inert. Pick

$$
\begin{aligned}
f & =\operatorname{char}\left(\left(\begin{array}{cc}
1+\varpi^{c} O_{F} & O_{F} \\
\varpi^{c} O_{F} & O_{F}
\end{array}\right)\right) \times \operatorname{char}\left(1+\varpi^{c} O_{F}\right), \\
f^{\prime} & =\sum_{a \in\left(O_{F} / \varpi^{c} O_{F}\right)^{*}}{ }^{\prime} \operatorname{char}\left(\left(\begin{array}{cc}
a^{-1}+\varpi^{c} O_{F} & O_{F} \\
\varpi^{c} O_{F} & O_{F}
\end{array}\right)\right) \times \operatorname{char}\left(a+\varpi^{c} O_{F}\right) \\
& =\sum_{a \in\left(O_{F} / \varpi^{c} O_{F}\right)^{*}} r^{\prime}\left(\left(\begin{array}{ll}
a & 0 \\
0 & 1
\end{array}\right)\right) f,
\end{aligned}
$$

where $\sum^{\prime}$ means the average. Pick $\Phi_{s}$ to be the unique up to constant $K_{1}\left(\varpi^{c}\right)$-invariant function supported on $B\left(\begin{array}{ll}1 & 0 \\ 1 & 1\end{array}\right) K_{1}\left(\varpi^{c}\right)$ such that $\Phi_{s}\left(\left(\begin{array}{ll}1 & 0 \\ 1 & 1\end{array}\right)\right)=1$. Then

$$
\mathbb{P}\left(s, w, f, \Phi_{s}\right)=\mathbb{P}\left(s, w, f^{\prime}, \Phi_{s}\right)=\frac{1}{\left(q^{2}-1\right)^{2} q^{4 c-4} \chi_{1, s}(\sqrt{D})} \frac{1}{1-q \delta \mu_{2} \chi_{1, s}} .
$$


Here $\delta=q^{-(w / 2+1 / 4)}$. When $w=1 / 2$, we have

$$
\mathbb{P}\left(s, 1 / 2, f^{\prime}, \Phi_{s}\right)=\frac{1}{\left(q^{2}-1\right)^{2} q^{4 c-4} \chi_{1}(\sqrt{D})} \frac{1}{1-\mu_{2} \chi_{1} q^{-(2 s+1 / 2)}} .
$$

The denominator is as expected since

$$
L_{v}(\Pi \otimes \Omega, 1 / 2) L_{v}\left(\left.\pi \otimes \chi_{1}\right|_{\mathbb{F}^{*}}, 2 s+1 / 2\right)=\frac{1}{1-\mu_{2} \chi_{1} q^{-(2 s+1 / 2)}}
$$

in this case, and

$$
\mathbb{P}^{0}\left(s, 1 / 2, f^{\prime}, \Phi_{s}\right)=\frac{1}{(q+1) q^{c-1} \chi_{1}(\sqrt{D})}
$$

First note that our choice for $f$ is only $K_{1}^{1}\left(\varpi^{c}\right)$-invariant under the Weil representation, where $K_{1}^{1}\left(\varpi^{c}\right)$ is the subgroup of $K$ whose elements are congruent to $\left(\begin{array}{ll}1 & * \\ 0 & 1\end{array}\right) \bmod \left(\varpi^{c}\right)$. On the other hand, $f^{\prime}$ is $K_{1}\left(\varpi^{c}\right)$-invariant under left, right, and Weil representation. For the following we will only compute $\mathbb{P}\left(s, w, f, \Phi_{s}\right)$. But all pieces in $f^{\prime}$ can be computed very similarly, and the result doesn't depend on $a$. Thus an average will give the same result.

Lemma 5.20

$$
G L_{2}=\coprod_{0 \leq i \leq c, \beta \in\left(O_{F} / \varpi^{\min \{i, c-i\}} O_{F}\right)^{*}} B\left(\begin{array}{cc}
1 & 0 \\
\varpi^{i} & 1
\end{array}\right)\left(\begin{array}{cc}
\beta & 0 \\
0 & 1
\end{array}\right) K_{1}^{1}\left(\varpi^{c}\right) .
$$

Proof First of all,

$$
K_{1}\left(\varpi^{c}\right)=\coprod_{\beta \in\left(O_{F} / \varpi^{c} O_{F}\right)^{*}}\left(\begin{array}{ll}
\beta & 0 \\
0 & 1
\end{array}\right) K_{1}^{1}\left(\varpi^{c}\right)
$$

We know by Lemma (2.1)

$$
\mathrm{GL}_{2}=\coprod_{0 \leq i \leq c} B\left(\begin{array}{cc}
1 & 0 \\
\varpi^{i} & 1
\end{array}\right) K_{1}\left(\varpi^{c}\right) \text {. }
$$

We just need to check when $B\left(\begin{array}{cc}1 & 0 \\ \varpi^{i} & 1\end{array}\right)\left(\begin{array}{ll}\beta & 0 \\ 0 & 1\end{array}\right) K_{1}^{1}\left(\varpi^{c}\right)=B\left(\begin{array}{cc}1 & 0 \\ \varpi^{i} & 1\end{array}\right)\left(\begin{array}{cc}\beta^{\prime} & 0 \\ 0 & 1\end{array}\right)$ $K_{1}^{1}\left(\varpi^{c}\right)$.

This is equvalent to that when modulo $\varpi^{c}$,

$$
\left(\begin{array}{cc}
1 & 0 \\
\varpi^{i} & 1
\end{array}\right)\left(\begin{array}{cc}
\beta / \beta^{\prime} & * \\
0 & 1
\end{array}\right)\left(\begin{array}{cc}
1 & 0 \\
-\varpi^{i} & 1
\end{array}\right)
$$

is upper triangular. That is

$$
\varpi^{i} \beta / \beta^{\prime}-\varpi^{i}-\varpi^{2 i} * \equiv 0 \bmod \left(\varpi^{c}\right) .
$$

Then the conclusion is clear.

We pick $\varphi \in \pi\left(\mu_{1}^{-1}, \mu_{2}^{-1}\right)$ to be the unique $K_{1}\left(\varpi^{c}\right)$-invariant function supported on $B K_{1}\left(\varpi^{c}\right)$. Then by the above lemma, the local integral $\mathbb{P}\left(s, w, f, \Phi_{s}\right)$ should be

$$
\begin{aligned}
& \sum_{0 \leq i \leq c, \beta} A_{i, \beta} \int W_{\varphi}^{-}\left(\left(\begin{array}{ll}
\alpha & 0 \\
0 & 1
\end{array}\right)\left(\begin{array}{cc}
1 & 0 \\
\varpi^{i} & 1
\end{array}\right)\right)|\alpha|^{\frac{w}{2}-\frac{1}{4}} \Phi_{s}(\alpha)^{-1} \\
& \quad \times I\left(\alpha, r^{\prime}\left(\left(\begin{array}{cc}
1 & 0 \\
\varpi^{i} & 1
\end{array}\right)\left(\begin{array}{ll}
\beta & 0 \\
0 & 1
\end{array}\right)\right) f, \Phi_{s}\right) d^{*} \alpha,
\end{aligned}
$$


where

$$
A_{i, \beta}=\frac{A_{i}}{\sharp\left(O_{F} / \varpi^{\min \{i, c-i\}} O_{F}\right)^{*}} .
$$

We shall work out the integral $I\left(\alpha, r^{\prime}\left(\left(\begin{array}{cc}1 & 0 \\ \varpi^{i} & 1\end{array}\right)\left(\begin{array}{ll}\beta & 0 \\ 0 & 1\end{array}\right)\right) f, \Phi_{s}\right)$ first. By (2.13),

$$
r^{\prime}\left(\left(\begin{array}{ll}
\beta & 0 \\
0 & 1
\end{array}\right)\right) f=\operatorname{char}\left(\left(\begin{array}{cc}
\beta^{-1}+\varpi^{c} O_{F} & O_{F} \\
\varpi^{c} O_{F} & O_{F}
\end{array}\right)\right) \times \operatorname{char}\left(\beta+\varpi^{c} O_{F}\right) .
$$

Then by Lemma 2.10 and the remark after it,

$$
\begin{aligned}
& r^{\prime}\left(\left(\begin{array}{cc}
1 & 0 \\
\varpi^{i} & 1
\end{array}\right)\left(\begin{array}{ll}
\beta & 0 \\
0 & 1
\end{array}\right)\right) f=q^{2(i-c)} \psi\left(u \varpi^{-i}\left[\left(x_{1}-\beta^{-1}\right) x_{4}-x_{2} x_{3}\right]\right) \\
& \times \operatorname{char}\left(\left(\begin{array}{cc}
\beta^{-1}+\varpi^{i} O_{F} & \varpi^{i-c} O_{F} \\
\varpi^{i} O_{F} & \varpi^{i-c} O_{F}
\end{array}\right)\right) \\
& \times \operatorname{char}\left(\beta+\varpi^{c} O_{F}\right) .
\end{aligned}
$$

Lemma 5.21

$$
\begin{aligned}
& \int \Phi_{s}\left(\gamma_{0} g\right) f\left(g, \frac{\alpha}{\operatorname{det} g}\right) d g= \begin{cases}\frac{1}{(q-1)\left(q^{2}-1\right) q^{3 c-3}} \chi_{1, s}\left(\frac{\alpha}{\sqrt{D}}\right) q^{\nu(\alpha)}, & \text { if } v(\alpha) \geq 0 \\
0, & \text { otherwise. }\end{cases} \\
& \int \Phi_{s}\left(\gamma_{0} g\right) r^{\prime}\left(\left(\begin{array}{cc}
1 & 0 \\
\varpi^{i} & 1
\end{array}\right)\left(\begin{array}{ll}
\beta & 0 \\
0 & 1
\end{array}\right)\right) f\left(g, \frac{\alpha}{\operatorname{det} g}\right) d g \equiv 0 \text { for any } i<c \text { and } \beta .
\end{aligned}
$$

Proof Recall that we can write $\mathrm{GL}_{2}=O_{E}^{*} B . \Phi_{s}\left(\gamma_{0} g\right)$ and $r^{\prime}\left(\left(\begin{array}{cc}1 & 0 \\ \varpi^{i} & 1\end{array}\right)\left(\begin{array}{ll}\beta & 0 \\ 0 & 1\end{array}\right)\right) f$ are both left invariant under $1+\varpi^{c} O_{E}$. Note that $O_{E}^{*} / 1+\varpi^{c} O_{E} \simeq\left(O_{E} / \varpi^{c} O_{E}\right)^{*}$ is of cardinality $\left(q^{2}-1\right) q^{2 c-2}$. Then

$$
\begin{aligned}
& I\left(\alpha, r^{\prime}\left(\left(\begin{array}{cc}
1 & 0 \\
\varpi^{i} & 1
\end{array}\right)\left(\begin{array}{cc}
\beta & 0 \\
0 & 1
\end{array}\right)\right) f, \Phi_{s}\right) \\
& =\frac{1}{\left(q^{2}-1\right) q^{2 c-2}} \sum_{t \in\left(O_{E} / \varpi^{c} O_{E}\right)^{*}} \int \Omega(t) \Phi_{s}\left(\gamma_{0}\left(\begin{array}{cc}
a_{1} & m \\
0 & a_{2}
\end{array}\right)\right) r^{\prime}\left(\left(\begin{array}{cc}
1 & 0 \\
\varpi^{i} & 1
\end{array}\right)\left(\begin{array}{cc}
\beta & 0 \\
0 & 1
\end{array}\right)\right) \\
& \quad \times f\left(t\left(\begin{array}{cc}
a_{1} & m \\
0 & a_{2}
\end{array}\right), \frac{\alpha}{N(t) a_{1} a_{2}}\right)\left|a_{2}\right|^{-1} d m d^{*} a_{1} d^{*} a_{2}
\end{aligned}
$$

If we write $t=b_{1}+b_{2} \sqrt{D}=\left(\begin{array}{cc}b_{1} & b_{2} \\ b_{2} D & b_{1}\end{array}\right)$, then $N(t)=b_{1}^{2}-b_{2}^{2} D$, and

$$
\Omega(t)=\chi_{1}(\bar{t}) \chi_{2}(t)=\chi_{1}\left(b_{1}-b_{2} \sqrt{D}\right)
$$

as $\chi_{2}$ is unramified.

First let $i=c$ and $\beta=1$. To satisfy

$$
t\left(\begin{array}{cc}
a_{1} & m \\
0 & a_{2}
\end{array}\right) \in\left(\begin{array}{cc}
1+\varpi^{c} O_{F} & O_{F} \\
\varpi^{c} O_{F} & O_{F}
\end{array}\right)
$$


we need $a_{1} b_{1} \in 1+\varpi^{c} O_{F}, a_{1} b_{2} D \in \varpi^{c} O_{F}$, and $a_{2}, m \in O_{F}$. If $b_{2} \notin \varpi^{c} O_{F}$, then it's impossible for $a_{1}$ to satisfy the first two conditions. Thus we only need to consider those $t$ with $b_{2} \in \varpi^{c} O_{F}, b_{1} \in\left(O_{F} / \varpi^{c} O_{F}\right)^{*}$. Then the domain for the integral is

$$
a_{1} \equiv b_{1}^{-1}+\varpi^{c} O_{F}, m \in O_{F} \text { and } a_{2} \in \frac{\alpha}{b_{1}}\left(1+\varpi^{c} O_{F}\right),
$$

as we also need $\frac{\alpha}{N(t) a_{1} a_{2}} \in 1+\varpi^{c} O_{F}$.

By Lemma 5.15,

$$
\Phi_{s}\left(\left(\begin{array}{cc}
1 & 0 \\
\sqrt{D} & 1
\end{array}\right)\left(\begin{array}{cc}
a_{1} & m \\
0 & a_{2}
\end{array}\right)\right)=\chi_{1, s}\left(\frac{a_{2}}{\sqrt{D}}\right) \chi_{2, s}\left(a_{1} \sqrt{D}\right),
$$

when the condition $v\left(a_{1}\right) \leq v\left(a_{2}+m \sqrt{D}\right)$ is satisfied. In particular over the domain in (5.94),

$$
\Phi_{s}= \begin{cases}\chi_{1, s}\left(\frac{\alpha}{b_{1} \sqrt{D}}\right), & \text { if } v(\alpha) \geq 0 \\ 0, & \text { otherwise }\end{cases}
$$

as $\chi_{2, s}$ is unramified and $v\left(a_{1} \sqrt{D}\right)=0$. Also $\Omega(t)=\chi_{1, s}\left(b_{1}-b_{2} \sqrt{D}\right)=\chi_{1, s}\left(b_{1}\right)$. Then for $v(\alpha) \geq 0$ the integral is easily computed to be

$$
\begin{aligned}
& \frac{1}{\left(q^{2}-1\right) q^{2 c-2}} \sum_{b_{1} \in\left(O_{F} / \varpi^{c} O_{F}\right)^{*}} \int_{\substack{a_{1} \equiv b_{1}^{-1}+\sigma^{c} O_{F} \\
m \in O_{F} \\
a_{2} \in \frac{\alpha}{b_{1}}\left(1+\varpi^{c} O_{F}\right)}} \chi_{1, s}\left(b_{1}\right) \chi_{1, s}\left(\frac{\alpha}{b_{1} \sqrt{D}}\right)\left|a_{2}\right|^{-1} d m d^{*} a_{1} d^{*} a_{2} \\
& =\frac{1}{(q-1)\left(q^{2}-1\right) q^{3 c-3}} \chi_{1, s}\left(\frac{\alpha}{\sqrt{D}}\right) q^{\nu(\alpha)}
\end{aligned}
$$

Now suppose $0<i<c$. For any fixed $t=b_{1}+b_{2} \sqrt{D}$ and $\beta$, we can do the integral similarly. In particular one would need $v\left(a_{1}\right) \leq v\left(a_{2}+m \sqrt{D}\right), b_{1} \in O_{F}^{*}$ and $b_{2} \in \varpi^{i} O_{F}$ for the integral to be nonzero. And when that's the case, the domain of the integral is

$$
v(m) \geq 0, \quad a_{2} \in \frac{b_{1} \alpha}{\left(b_{1}^{2}-b_{2}^{2} D\right)}\left(1+\varpi^{i} O_{F}\right) \quad \text { and } \quad a_{1} \in \frac{\beta^{-1} \alpha}{\left(b_{1}^{2}-b_{2}^{2} D\right) a_{2}}\left(1+\varpi^{c} O_{F}\right) .
$$

Over this domain, we have

$$
\begin{aligned}
\psi\left(u \varpi^{-i}\left[\left(x_{1}-\beta^{-1}\right) x_{4}-x_{2} x_{3}\right]\right) & =\psi\left(\varpi^{-i} \alpha\right) \psi\left(-\frac{\beta^{-1} \alpha \varpi^{-i}}{\left(b_{1}^{2}-b_{2}^{2} D\right) a_{1} a_{2}}\left(a_{2} b_{1}+m b_{2} D\right)\right) \\
& =\psi\left(\varpi^{-i} \alpha\right) \psi\left(-\frac{b_{1}^{2} \alpha \varpi^{-i}}{b_{1}^{2}-b_{2}^{2} D}\right),
\end{aligned}
$$

which turns out to be constant over the domain.

Now we do the integral of $\chi_{1, s}\left(\frac{a_{2}}{\sqrt{D}}\right) \chi_{2, s}\left(a_{1} \sqrt{D}\right)$ over the above domain. When integrating in $a_{1}$ first, we are essentially integrating a constant as $\chi_{2, s}$ is unramified. Then the integral in $a_{2}$ is essentially

$$
\int_{a_{2} \in \frac{b_{1} \alpha}{\left(b_{1}^{2}-b_{2}^{2} D\right)}\left(1+\varpi^{i} O_{F}\right)} \chi_{1, s}\left(a_{2}\right) d^{*} a_{2},
$$

which is zero according to Lemma 2.4 .

When $i=0$, the proof is similar. We will leave this case to the reader. 
According to this Lemma, we only need to compute one integral for (5.88) and we only care about $W_{\varphi}^{-}\left(\left(\begin{array}{ll}\alpha & 0 \\ 0 & 1\end{array}\right)\right)$.

Lemma 5.22 Assume that $\mu_{1}$ is unramified and $\mu_{2}$ is of level $c>0$. Suppose that $\varphi \in$ $\pi\left(\mu_{1}^{-1}, \mu_{2}^{-1}\right)$ is the unique $K_{1}\left(\varpi^{c}\right)-$ invariant function supported on $B K_{1}\left(\varpi^{c}\right)$. Then

$$
W_{\varphi}^{-}\left(\left(\begin{array}{ll}
\alpha & 0 \\
0 & 1
\end{array}\right)\right)= \begin{cases}q^{-v(\alpha) / 2} \mu_{1}^{-v(\alpha)}, & \text { if } v(\alpha) \geq 0 \\
0, & \text { if } v(\alpha)<0 .\end{cases}
$$

Proof By Lemma 5.6, in particular by part (2ii), we have

$$
\varphi\left(\omega\left(\begin{array}{cc}
1 & m \\
0 & 1
\end{array}\right)\left(\begin{array}{cc}
\alpha & 0 \\
0 & 1
\end{array}\right)\right)=\mu_{1}^{-1}\left(-\frac{\alpha}{m}\right) \mu_{2}^{-1}(-m)\left|\frac{\alpha}{m^{2}}\right|^{1 / 2},
$$

when $v(m) \leq v(\alpha)-c$. Recall that $W_{\varphi}^{-}$is the Whittaker function for $\varphi$ associated to $\psi^{-}$. Then

$$
\begin{aligned}
W_{\varphi}^{-}\left(\left(\begin{array}{ll}
\alpha & 0 \\
0 & 1
\end{array}\right)\right) & =\int_{\nu(m) \leq \nu(\alpha)-c} \mu_{1}^{-1}\left(-\frac{\alpha}{m}\right) \mu_{2}^{-1}(-m)\left|\frac{\alpha}{m^{2}}\right|^{1 / 2} \psi(m) d m \\
& = \begin{cases}C^{\prime} q^{-\nu(\alpha) / 2} \mu_{1}^{-v(\alpha)}, & \text { if } v(\alpha) \geq 0 ; \\
0, & \text { if } v(\alpha)<0 .\end{cases}
\end{aligned}
$$

where

$$
C^{\prime}=\frac{1}{q^{c} \mu_{1}^{c}} \int_{\nu(m)=-c} \mu_{2}^{-1}(-m) \psi(m) d m
$$

is a non-zero constant and will be cancelled after normalization.

Now we combine Lemma 5.21 and 5.22 into (5.88). One can easily see that

$$
\begin{aligned}
\mathbb{P}\left(s, w, f, \Phi_{s}\right)= & A_{c} \int_{\nu(\alpha) \geq 0} q^{-v(\alpha) / 2} \mu_{1}^{-v(\alpha)}|\alpha|^{\frac{w}{2}-\frac{1}{4}} \Phi_{s}(\alpha)^{-1} \\
& \times \frac{1}{(q-1)\left(q^{2}-1\right) q^{3 c-3}} \chi_{1, s}\left(\frac{\alpha}{\sqrt{D}}\right) q^{\nu(\alpha)} d^{*} \alpha \\
= & \frac{1}{\left(q^{2}-1\right)^{2} q^{4 c-4} \chi_{1, s}(\sqrt{D})} \frac{1}{1-q \delta \mu_{2} \chi_{1, s}} .
\end{aligned}
$$

Here $\delta=q^{-(w / 2+1 / 4)}$. We have used that $\mu_{1} \mu_{2} \chi_{1, s} \chi_{2, s}=1$ and $\mu_{2} \chi_{1, s}$ is unramified. When $w=1 / 2$, we have

$$
\mathbb{P}\left(s, 1 / 2, f, \Phi_{s}\right)=\frac{1}{\left(q^{2}-1\right)^{2} q^{4 c-4} \chi_{1, s}(\sqrt{D})} \frac{1}{1-\mu_{2} \chi_{1} q^{-(2 s+1 / 2)}} .
$$

To compute $\left\langle F_{1}, F\right\rangle$, we shall compute the corresponding integral using $f^{\prime}$.

$$
\begin{aligned}
\left\langle F_{1}, F\right\rangle= & \sum_{0 \leq i \leq c, \beta} A_{i} \iint W_{\varphi}^{-}\left(\left(\begin{array}{cc}
\alpha & 0 \\
0 & 1
\end{array}\right)\left(\begin{array}{cc}
1 & 0 \\
\varpi^{i} & 1
\end{array}\right)\right) r^{\prime}\left(\left(\begin{array}{cc}
1 & 0 \\
\varpi^{i} & 1
\end{array}\right)\right) \\
& \times f^{\prime}\left(\alpha x, \alpha^{-1} x^{-2}\right)\left(\mu_{1} \mu_{2}\right)^{-1}(x) d^{*} x d^{*} \alpha .
\end{aligned}
$$


By Formula (5.90), we have for any $i$,

$$
\begin{aligned}
r^{\prime}\left(\left(\begin{array}{cc}
1 & 0 \\
\varpi^{i} & 1
\end{array}\right)\right) f^{\prime}= & \sum_{a \in\left(O_{F} / \varpi^{c} O_{F}\right)^{*}} q^{2(i-c)} \psi\left(u \varpi^{-i}\left[\left(x_{1}-a^{-1}\right) x_{4}-x_{2} x_{3}\right]\right) \\
& \times \operatorname{char}\left(\left(\begin{array}{cc}
a^{-1}+\varpi^{i} O_{F} & \varpi^{i-c} O_{F} \\
\varpi^{i} O_{F} & \varpi^{i-c} O_{F}
\end{array}\right)\right) \times \operatorname{char}\left(a+\varpi^{c} O_{F}\right)
\end{aligned}
$$

$\left(\alpha x, \alpha^{-1} x^{-2}\right)$ is in the support if and only if $x \in 1+\varpi^{i} O_{F}$. On the support, it's a constant function in $x$. Then integral against $\left(\mu_{1} \mu_{2}\right)(x)$ is nonzero if and only if $i=c$. Thus

$$
\begin{aligned}
\left\langle F_{1}, F\right\rangle & =A_{c} \iint W_{\varphi}^{-}\left(\left(\begin{array}{ll}
\alpha & 0 \\
0 & 1
\end{array}\right)\right) f^{\prime}\left(\alpha x, \alpha^{-1} x^{-2}\right)\left(\mu_{1} \mu_{2}\right)^{-1}(x) d^{*} x d^{*} \alpha \\
& =A_{c} \int_{v(\alpha)=0} W_{\varphi}^{-}\left(\left(\begin{array}{ll}
\alpha & 0 \\
0 & 1
\end{array}\right)\right) \frac{1}{\left(q-1^{2}\right)\left(q^{2 c-2}\right)} d^{*} \alpha \\
& =\frac{1}{(q-1)\left(q^{2}-1\right)\left(q^{3 c-3}\right)} .
\end{aligned}
$$

The expected L-factors in this case is

$$
\frac{\zeta(2)}{L(\pi, A d, 1)} \frac{L(\Pi \otimes \Omega, 1 / 2) L\left(\left.\pi \otimes \chi_{1}\right|_{\mathbb{F}^{*},} 2 s+1 / 2\right)}{L(\eta, 1) L(\chi, 2 s+1)}=\frac{1}{1-\mu_{2} \chi_{1} q^{-(2 s+1 / 2)}}
$$

Thus

$$
\mathbb{P}^{0}\left(s, 1 / 2, f, \Phi_{s}\right)=\frac{1}{(q+1) q^{c-1} \chi_{1}(\sqrt{D})} .
$$

\subsection{The last case when only $\pi$ is ramified}

In this section, we consider a finite place where $\pi$ is highly ramified, $\chi_{1}$ and $\chi_{2}$ are unramified and $\mathbb{E} / \mathbb{F}$ is inert. As mentioned in the end of Section 3, the local integral of our problem can also be formulated in terms of matrix coefficients:

$$
\int_{\mathbb{F}^{*} \backslash \mathrm{GL}_{2}(\mathbb{F})} \Phi_{s}\left(\gamma_{0} g\right)\left\langle F_{1}, \pi(g) F\right\rangle d g,
$$

where $F_{1} \in \hat{\pi}, F \in \pi$ and $\langle\cdot, \cdot\rangle$ is a bilinear and $\mathrm{GL}_{2}(\mathbb{F})$ - invariant pairing between $\hat{\pi}$ and $\pi$.

If the level $c$ of the representation $\pi$ is odd, then the local integral is automatically zero according to Theorem 2.14 and Example 2.15. Assume from now on that $c=2 k$.

Define $\tilde{K}$ to be the subgroup of $\mathrm{GL}_{2}\left(O_{E}\right)$ whose elements are congruent to $\left(\begin{array}{ll}1 & 0 \\ 0 & 1\end{array}\right)$ $\bmod \left(\varpi^{k} O_{E}\right)$. Define $\Phi_{s}$ to be the unique up to constant function from the induced representation such that it's right $\tilde{K}$-invariant and supported on $B\left(\begin{array}{cc}0 & 1 \\ -1 & -\frac{\sqrt{D}}{D}\end{array}\right) \tilde{K}$. It will be normalized such that

$$
\Phi_{s}\left(\left(\begin{array}{cc}
0 & 1 \\
-1 & -\frac{\sqrt{D}}{D}
\end{array}\right)\right)=1 .
$$


As motivated by Example 2.18, we will pick $F_{1}$ and $F$ to be the unique up to constant elements from respective representations which are invariant under

$$
\left\{\left(\begin{array}{cc}
a+\varpi^{k} O_{F} & b+\varpi^{k} O_{F} \\
b D+\varpi^{k} O_{F} & a+\varpi^{k} O_{F}
\end{array}\right) \mid a+b \sqrt{D} \in O_{E}^{*}\right\} .
$$

Let $F_{1}$ and $F$ be so normalized that

$$
\left\langle F_{1}, F\right\rangle=1
$$

Proposition 5.23 Suppose that $\pi$ is highly ramified of level $c=2 k, \chi_{1}$ and $\chi_{2}$ are both unramified, and $\mathbb{E} / \mathbb{F}$ is inert. Then for the choice of $F_{1} \in \hat{\pi}, F \in \pi$ and $\Phi_{s}$ as given above, we have

$$
\int_{\mathbb{F}^{*} \backslash G L_{2}(\mathbb{F})} \Phi_{s}\left(\gamma_{0} g\right)\left\langle F_{1}, \pi(g) F\right\rangle d g=\frac{1}{(q-1) q^{c-1}},
$$

and

$$
\mathbb{P}^{0}=\frac{L(\pi, A d, 1)}{q^{c}\left(1-\chi q^{-(4 s+2)}\right)}
$$

We first give the property for $\Phi_{s}$.

Lemma 5.24 Let $\Phi_{s}$ be the unique normalized element from the induced representation which is supported on $B\left(\begin{array}{cc}0 & 1 \\ -1 & -\frac{\sqrt{D}}{D}\end{array}\right) \tilde{K}$. Then

$$
\Phi_{s}\left(\gamma_{0}\left(\begin{array}{cc}
a_{1} & m \\
0 & 1
\end{array}\right)\right)= \begin{cases}1, & \text { if } v(m) \geq k \text { and } a_{1} \equiv 1 \quad \bmod \left(\varpi^{k} O_{F}\right) \\
0, & \text { otherwise }\end{cases}
$$

Proof Let's consider when the matrix $\left(\begin{array}{cc}1 & 0 \\ \sqrt{D} & 1\end{array}\right)\left(\begin{array}{cc}a_{1} & m \\ 0 & 1\end{array}\right)$ can be in the support $B\left(\begin{array}{cc}0 & 1 \\ -1 & -\frac{\sqrt{D}}{D}\end{array}\right) \tilde{K}$. This is equivalent to say if there exists $k \in \tilde{K}$ such that

$$
\left(\begin{array}{cc}
a_{1} & m \\
a_{1} \sqrt{D} & 1+m \sqrt{D}
\end{array}\right) k\left(\begin{array}{cc}
-\frac{\sqrt{D}}{D} & -1 \\
1 & 0
\end{array}\right)
$$

is upper triangular. This in turn is equivalent to that

$$
-a_{1}+1+m \sqrt{D} \equiv 0 \quad \bmod \left(\varpi^{k} O_{E}\right)
$$

Thus one get the conditions for $\Phi_{s}\left(\gamma_{0}\left(\begin{array}{cc}a_{1} & m \\ 0 & 1\end{array}\right)\right)$ to be non-zero as in the lemma. When these conditions are satisfied, the rest are easy to check.

Now we can prove Proposition 5.23 easily. As $\chi_{1}$ and $\chi_{2}$ are unramified, $\Phi_{s}\left(\gamma_{0} t g\right)=$ $\Phi_{s}\left(\gamma_{0} g\right)$ for $t \in O_{E}^{*}$. Note that $\mathbb{F}^{*} \backslash G L_{2}=O_{E}^{*}\left\{\left(\begin{array}{cc}a_{1} & m \\ 0 & 1\end{array}\right)\right\}$. Then the local integral (5.107) becomes 


$$
\begin{aligned}
& \int_{\mathbb{F}^{*} \backslash \mathrm{GL}_{2}(\mathbb{F})} \Phi_{s}\left(\gamma_{0} g\right)\left\langle F_{1}, \pi(g) F\right\rangle d g \\
= & \int_{t \in O_{E}^{*}} \int_{a_{1}, m} \Omega(t) \Phi_{s}\left(\gamma_{0}\left(\begin{array}{cc}
a_{1} & m \\
0 & 1
\end{array}\right)\right)\left\langle\hat{\pi}\left(t^{-1}\right) F_{1}, \pi\left(\left(\begin{array}{cc}
a_{1} & m \\
0 & 1
\end{array}\right)\right) F\right\rangle d^{*} a_{1} d m d^{*} t \\
= & \int_{a_{1}, m} \Phi_{s}\left(\gamma_{0}\left(\begin{array}{cc}
a_{1} & m \\
0 & 1
\end{array}\right)\right)\left\langle F_{1}, \pi\left(\left(\begin{array}{cc}
a_{1} & m \\
0 & 1
\end{array}\right)\right) F\right\rangle d^{*} a_{1} d m \\
= & \int_{v(m) \geq k \text { and } a_{1} \equiv 1 \bmod \left(\varpi^{k}\right)}\left\langle F_{1}, \pi\left(\left(\begin{array}{cc}
a_{1} & m \\
0 & 1
\end{array}\right)\right) F\right\rangle d^{*} a_{1} d m .
\end{aligned}
$$

Here we have used the fact that $F_{1}$ is invariant under $O_{E}^{*}$ for the second equality, and Lemma 5.24 for the last equality.

Note that when $v(m) \geq k$ and $a_{1} \equiv 1 \bmod \left(\varpi^{k}\right)$,

$$
\left(\begin{array}{cc}
a_{1} & m \\
0 & 1
\end{array}\right) \in\left\{\left(\begin{array}{cc}
a+\varpi^{k} O_{F} & b+\varpi^{k} O_{F} \\
b D+\varpi^{k} O_{F} & a+\varpi^{k} O_{F}
\end{array}\right) \mid a+b \sqrt{D} \in O_{E}^{*}\right\},
$$

under the action of which $F$ is invariant.

Thus

$$
\begin{aligned}
& \int_{\mathbb{F}^{*} \backslash \mathrm{GL}_{2}(\mathbb{F})} \Phi_{s}\left(\gamma_{0} g\right)\left\langle F_{1}, \pi(g) F\right\rangle d g \\
= & \int_{v(m) \geq k \text { and } a_{1} \equiv 1 \bmod \left(\varpi^{k}\right)}\left\langle F_{1}, F\right\rangle d^{*} a_{1} d m=\frac{1}{(q-1) q^{c-1}} .
\end{aligned}
$$

The expected L-factors in this case is

$$
\begin{aligned}
& \frac{\zeta(2)}{L(\pi, A d, 1)} \frac{L(\Pi \otimes \Omega, 1 / 2) L\left(\left.\pi \otimes \chi_{1}\right|_{\mathbb{F}^{*}}, 2 s+1 / 2\right)}{L(\eta, 1) L(\chi, 2 s+1)} \\
& \quad=\frac{1}{\left(1-q^{-2}\right) L(\pi, A d, 1)}\left(1+q^{-1}\right)\left(1-\chi q^{-(4 s+2)}\right) .
\end{aligned}
$$

So

$$
\mathbb{P}^{0}\left(s, 1 / 2, f, \Phi_{s}\right)=\frac{L(\pi, A d, 1)}{q^{c}\left(1-\chi q^{-(4 s+2)}\right)} .
$$

\section{Archimedean places}

The local integral at archimedean places in general can be very complicated to compute. In this section we shall restrict ourselves to the following setting. Suppose that $\mathbb{E}$ is totally real. In particular all infinity places of $\mathbb{F}$ are real and they split in $\mathbb{E}$. Suppose that the cusp form $F$ is anti-holomorphic and is of parallel weight $-2 n$, and the Eisenstein series is holomorphic and is of parallel weight $n$.

In terms of local components, we assume that $\pi$ is of form $\sigma\left(|\cdot|^{\frac{2 n-1}{2}},|\cdot|^{-\frac{2 n-1}{2}}\right)$. Pick $F_{1}$ and $F$ to be weight $-2 n$ elements. It is well-known that in general the matrix coefficient associated to holomorphic weight $2 n>0$ elements is

$$
\varphi\left(\left(\begin{array}{cc}
a & m \\
0 & 1
\end{array}\right)\right)=\frac{a^{n}(2 i)^{2} n}{(m+(a+1) i)^{2} n}
$$


Note that it is normalized so that $\varphi(1)=1$. Then the matrix coefficient associated to weight $-2 n$ elements is

$$
\varphi\left(\left(\begin{array}{cc}
a & m \\
0 & 1
\end{array}\right)\right)=\frac{a^{n}(2 i)^{2} n}{(-m+(a+1) i)^{2} n} .
$$

Here we have conjugated the formula by $\left(\begin{array}{cc}-1 & 0 \\ 0 & 1\end{array}\right)$ to get the matrix coefficient for weight $-2 n$ elements.

Since this real place splits in $\mathbb{E}$, we shall write $\Phi_{s}=\Phi_{s}^{(1)} \Phi_{s}^{(2)}$, where we pick $\Phi_{s}^{(i)}$ to be the weight $n$ element from $\operatorname{Ind}_{B}^{\mathrm{GL}_{2}}\left(\operatorname{sgn}^{\delta}(\cdot)|\cdot|^{\frac{n-1}{2}},|\cdot|^{-\frac{n-1}{2}}\right)$. Here $\delta$ is 0 if $n$ is even and 1 if $n$ is odd. We shall think $\sqrt{D}=(\sqrt{D},-\sqrt{D})$ in this case. Recall that the local integral is

$$
\mathbb{P}=\int_{\mathbb{F}^{*} \backslash \mathrm{GL}_{2}(\mathbb{F})}\left\langle F_{1}, \pi(g) F\right\rangle \Phi_{s}\left(\gamma_{0} g\right) d g=\int_{\mathbb{F}^{*} \backslash \mathrm{GL}_{2}(\mathbb{F})} \varphi(g) \Phi_{s}\left(\gamma_{0} g\right) d g .
$$

Here we shall use the decomposition

$$
\mathbb{F}^{*} \backslash \mathrm{GL}_{2}(\mathbb{F})=\left\{\left(\begin{array}{cc}
a & m \\
0 & 1
\end{array}\right) \mid a \in \mathbb{R}^{*}, m \in \mathbb{R}\right\} K
$$

for $K=S O(2)$, and the Haar measure is

$$
\frac{1}{2 \pi} \frac{d a d m d \theta}{a^{2}} \text {. }
$$

Note that

$$
\begin{aligned}
\left(\begin{array}{cc}
a & m \\
\pm a \sqrt{D} & 1 \pm m \sqrt{D}
\end{array}\right)= & \left(\begin{array}{cc}
\frac{a}{\sqrt{a^{2} D+(1 \pm m \sqrt{D})^{2}}} & \frac{ \pm a^{2} \sqrt{D}+m \pm m^{2} \sqrt{D}}{\sqrt{a^{2} D+(1 \pm m \sqrt{D})^{2}}} \\
0 & \sqrt{a^{2} D+(1 \pm m \sqrt{D})^{2}}
\end{array}\right) \\
& \times\left(\begin{array}{ll}
\frac{1 \pm m \sqrt{D}}{\sqrt{a^{2} D+(1 \pm m \sqrt{D})^{2}}} & \frac{\mp a \sqrt{D}}{\sqrt{a^{2} D+(1 \pm m \sqrt{D})^{2}}} \\
\frac{ \pm a \sqrt{D}}{\sqrt{a^{2} D+(1 \pm m \sqrt{D})^{2}}} & \frac{1 \pm m \sqrt{D}}{\sqrt{a^{2} D+(1 \pm m \sqrt{D})^{2}}}
\end{array}\right)
\end{aligned}
$$

So by definition,

$$
\begin{aligned}
\mathbb{P}= & \int_{a>0} \int_{m} \frac{a^{n} 2^{2 n}}{(-m+(a+1) i)^{2 n}}\left(\frac{a}{a^{2} D+(1+m \sqrt{D})^{2}}\right)^{\frac{n}{2}}\left(\frac{1+m \sqrt{D}-i a \sqrt{D}}{\sqrt{a^{2} D+(1+m \sqrt{D})^{2}}}\right)^{n} \\
& \times\left(\frac{a}{a^{2} D+(1-m \sqrt{D})^{2}}\right)^{\frac{n}{2}}\left(\frac{1-m \sqrt{D}+i a \sqrt{D}}{\sqrt{a^{2} D+(1-m \sqrt{D})^{2}}}\right)^{n} a^{-2} d a d m
\end{aligned}
$$

We have used that $\varphi$ is only supported on $\operatorname{det}(g)>0$. It can be easily simplified as

$$
\mathbb{P}=\frac{2^{2 n}(-1)^{n}}{D^{n}} \int_{a>0} \int_{m} \frac{a^{2 n-2}}{(m-(a+1) i)^{2 n}\left(m+\frac{1}{\sqrt{D}}+i a\right)^{n}\left(m-\frac{1}{\sqrt{D}}+i a\right)^{n}} d a d m .
$$

As an analytic function in $m$, the integrand has a pole of order $2 n$ at $m=(a+1) i$ in the upper half plane, and two poles of order $n$ at $m= \pm \frac{1}{\sqrt{D}}-i a$ in the lower half plane. To fully make use of the symmetry, we shall shift the contour integral upward and use basic complex analysis.

$$
\mathbb{P}=\left.\frac{2 \pi i}{(2 n-1) !} \frac{2^{2 n}(-1)^{n}}{D^{n}} \int_{a>0} a^{2 n-2}\left(\frac{d}{d m}\right)^{2 n-1}\left(\frac{1}{\left((m+i a)^{2}-\frac{1}{D}\right)^{n}}\right)\right|_{m=(a+1) i} d a
$$


Now we make use of the linear relation between $m$ and $a$ in the expression, and do the following change of variable:

$$
u=\frac{m+i a-i}{2 i}
$$

Then

$$
\begin{aligned}
& \left.\left(\frac{d}{d m}\right)^{2 n-1}\left(\frac{1}{\left((m+i a)^{2}-\frac{1}{D}\right)^{n}}\right)\right|_{m=(a+1) i} \\
& \quad=\left.\left(\frac{1}{2 i}\right)^{2 n-1}\left(\frac{d}{d u}\right)^{2 n-1}\left(\frac{1}{\left((2 u+1)^{2}+\frac{1}{D}\right)^{n}(-1)^{n}}\right)\right|_{u=a} .
\end{aligned}
$$

So

$$
\mathbb{P}=-\left.\frac{4 \pi}{(2 n-1) ! D^{n}} \int_{a>0} a^{2 n-2}\left(\frac{d}{d u}\right)^{2 n-1}\left(\frac{1}{\left((2 u+1)^{2}+\frac{1}{D}\right)^{n}}\right)\right|_{u=a} d a .
$$

Now we can use integration by parts multiple times to get

$$
\begin{aligned}
\mathbb{P}= & -\frac{4 \pi}{(2 n-1) ! D^{n}}\left[\left.a^{2 n-2}\left(\frac{d}{d a}\right)^{2 n-2}\left(\frac{1}{\left((2 a+1)^{2}+\frac{1}{D}\right)^{n}}\right)\right|_{a=0} ^{\infty}\right. \\
& -\left.(2 n-2) a^{2 n-3}\left(\frac{d}{d a}\right)^{2 n-3}\left(\frac{1}{\left((2 a+1)^{2}+\frac{1}{D}\right)^{n}}\right)\right|_{a=0} ^{\infty}+\cdots \\
& \left.+\left.(2 n-2) !\left(\frac{1}{\left((2 a+1)^{2}+\frac{1}{D}\right)^{n}}\right)\right|_{a=0} ^{\infty}\right] \\
= & \frac{4 \pi}{(2 n-1) ! D^{n}}(2 n-2) ! \frac{1}{\left(1+\frac{1}{D}\right)^{n}}=\frac{4 \pi}{2 n-1} \frac{1}{(1+D)^{n}} .
\end{aligned}
$$

Here we have used that $\left.a^{i}\left(\frac{d}{d a}\right)^{i}\left(\frac{1}{\left((2 a+1)^{2}+\frac{1}{D}\right)^{n}}\right)\right|_{a=0} ^{\infty}=0$ for $0<i \leq 2 n-2$. We will not normalize the local integral by L-factors for archimedean places.

\section{Acknowledgements}

I would like to thank my advisor, Prof. Tonghai Yang. He suggested this problem to me and has given me guidance throughout this paper. I would like to thank Robert Harron, Dipendra Prasad, Michael Woodbury and Lei Zhang for helpful discussions. I would also like to thank referee for suggestions to improve the paper. This paper is partially supported by Graduate School Grant and NSF Grant of Prof. Tonghai Yang.

\section{Appendix A: Kirillov model for the supercuspidal representation and its newform}

Let $\mathbb{F}_{v}$ be a local field and $p$ be the characteristic of its residue field. Let $\psi$ be an unramified additive character. Let $\pi$ be a supercuspidal representation over $\mathbb{F}_{v}$, with central character $w_{\pi}$. Its Kirillov model can be realized on $S\left(\mathbb{F}^{*}\right)$ such that

$$
\pi\left(\left(\begin{array}{cc}
a_{1} & m \\
0 & a_{2}
\end{array}\right)\right) \varphi(x)=w_{\pi}\left(a_{2}\right) \psi\left(m a_{2}^{-1} x\right) \varphi\left(a_{1} a_{2}^{-1} x\right)
$$


A basis of this representation can be given by

$$
\mathbf{1}_{v, n}(x)= \begin{cases}v(u), & \text { if } x=u \varpi^{n} \text { for } u \in O_{F}^{*} \\ 0, & \text { otherwise. }\end{cases}
$$

The action of $\omega=\left(\begin{array}{cc}0 & 1 \\ -1 & 0\end{array}\right)$ on this basis is given by

$$
\pi(\omega) \mathbf{1}_{v, n}=C_{v w_{0}^{-1}} z_{0}^{-n} \mathbf{1}_{v^{-1} w_{0},-n+n_{v}-1},
$$

where $z_{0}=w_{\pi}(\varpi)$ and $w_{0}=\left.w_{\pi}\right|_{O_{F}^{*}}$. Recall that $n_{v} \leq-2$ and $c=-n_{1}$.

For simplicity, we focus on the case when $w_{\pi}$ is unramified or level 1. Correspondingly $w_{0}=\left.w_{\pi}\right|_{O_{F}^{*}}$ is trivial or level 1 .

Proposition A.1 Suppose $p \neq 2$. Let $c \geq 2$ be the level of a supercuspidal representation $\pi$ whose central character is unramified or level 1 . If $v$ is a level $i$ character of $O_{F}^{*}$, then we have

$$
n_{v}=\min \{-c,-2 i\}
$$

Proof Note that

$$
\omega\left(\begin{array}{cc}
1 & -1 \\
0 & 1
\end{array}\right) \omega=\left(\begin{array}{ll}
1 & 1 \\
0 & 1
\end{array}\right) \omega\left(\begin{array}{ll}
1 & 1 \\
0 & 1
\end{array}\right)
$$

We will just apply this equation to different test functions and compare levels or supports of each side. First of all, consider $\mathbf{1}_{1, n}$ for $n \geq 0$. By the classical result of Gauss sum, the right hand side of (A.3) will give a linear combination of $\mathbf{1}_{v,-n+n_{1}}$ for all $v$ of level $-n_{1}+n$. The left hand side has an additional action of $\omega$, which has to maintain the right hand side. In particular,

$$
n_{v}=2 n_{1}-2 n
$$

for all $v$ of level $-n_{1}+n$.

Then we consider those $v$ of level from 1 to $-n_{1}-1$. Suppose $1 \leq i<c / 2$.

First we test $\mathbf{1}_{1,-i}$. The situation for the left hand side is similar. The action of $\omega$ will change $\mathbf{1}_{1,-i}$ into a multiple of $\mathbf{1}_{w_{0}, i-c}$. Then $\psi(-x) \mathbf{1}_{w_{0}, i-c}$ will be a linear combination of $\mathbf{1}_{w_{0} v, i-c}$ for all $v$ of level $c-i$. After another action of $\omega$, what we get just consists of all level $c-i$ characters.

On the right hand side, $\psi \mathbf{1}_{1,-i}$ consists of all $\mathbf{1}_{\mu,-i}$ for $\mu$ of level $i$. If $i=1$, it should be understood that $\mu$ is of level 1 or 0 . If $n_{\mu}>-c$ for some $\mu$ of level $i$, then the action of $\omega$ will change $\mathbf{1}_{\mu^{-1},-i}$ into $\mathbf{1}_{\mu w_{0}^{-1}, i+n_{\mu}}$, so we know $\pi\left(\omega\left(\begin{array}{ll}1 & 1 \\ 0 & 1\end{array}\right) \mathbf{1}_{1,-i}\right.$ has level $i<c-i$ components at $v(x)=i+n_{\mu}>i-c$. Note that $\mu$ and $\psi(x)$ at $v(x)=i+n_{\mu}$ are both of level $<c-i$. Then multiplying another $\psi(x)$ will never give level $c-i$ components at $v(x)=i+n_{\mu}$, contradiction. So $n_{v} \leq-c$.

As a direct result of this, we also get $n_{v} \leq-2(c-i)$ for all $v$ of level $c-i$. This is because in our argument for $n_{\mu} \leq-c$, the right hand side will be supported at $v(x) \leq i-c$. Then on the left hand side, the action of $\omega$ has to change all level $c-i$ components at $v(x)=i-c$ back to $v(x) \leq i-c$.

Now if $n_{\mu^{\prime}}=n_{\mu^{\prime-1} w_{0}^{-1}}<-c$ for some character $\mu^{\prime}$ of level $i$, then we can test on $\mathbf{1}_{\mu^{\prime-1},-i}$. The left hand side will give purely level $-n_{\mu^{\prime}}-i>c-i$ components, supported 
at $v(x) \leq i+n_{\mu^{\prime}}$ by what we just showed above. On the right hand side we still have level 0 component in $\psi \mathbf{1}_{\mu^{\prime},-i}$ as $\psi(x)$ at $v(x)=-i$ has $\mu^{\prime-1}$ component. The action of $\left(\begin{array}{ll}1 & 1 \\ 0 & 1\end{array}\right) \omega$ on this level 0 component will give a nonzero part at $v(x)=i+n_{1}=i-c$. This contradicts the support of the left hand side. So $n_{\mu} \geq-c$ for all $\mu$ of level $i$.

Combine the two arguments above, we can conclude that when $1 \leq i<c / 2$,

$$
n_{\mu}=-c
$$

for all $\mu$ of level $i$, and

$$
n_{v}=-2(c-i)
$$

for all $v$ of level $c-i$.

Now we only have to consider the middle level characters. Suppose that $c$ is even and $v$ is a character of level $c / 2$. The expected value of $n_{v}$ is still $-c$, as suggested by the statement of the lemma. While the argument above to prove $n_{v} \geq-c$ still works, the argument about $n_{v} \leq-c$ will fail. Indeed $\pi\left(\omega\left(\begin{array}{ll}1 & 1 \\ 0 & 1\end{array}\right)\right) \mathbf{1}_{1,-i}$ has level $i=c-i$ components. Note that for $c=2, n_{v} \leq-2$ is automatic, so we can assume $c \geq 4$. We need a more accurate description of each side and try to compare the support in addition to the levels.

We know $n_{v} \geq-c$ for all $v$ of level $c / 2 \geq 2$. Suppose that $n_{v^{\prime}}>-c$ is the largest among all characters of level $c / 2$. (Recall that $n_{v} \leq-2$ for any $v$.) Choose the test function to be $\mathbf{1}_{v^{\prime-1},-c / 2}$. The action of $\omega$ will change it into a multiple of $\mathbf{1}_{v^{\prime} w_{0}, c / 2+n_{v^{\prime}}}$. Then $\psi(-x) \mathbf{1}_{v^{\prime} w_{0}, c / 2+n_{v^{\prime}}}$ consists of level $c / 2$ characters that differ from $v^{\prime} w_{0}$ by smaller level characters which are components of $\psi(-x)$ for $v(x)=c / 2+n_{v^{\prime}}>-c / 2$. That is, it's a linear combination of $\mathbf{1}_{v^{\prime} w_{0} v, c / 2+n_{v^{\prime}}}$ for $v$ of level less than $c / 2$ introduced by $\psi(-x)$. When changed back by another $\omega$ action, what we get is a linear combination of characters $v^{\prime-1} v^{-1}$. They are supported on $v(x) \leq-c / 2$, as we have assumed $n_{v^{\prime}}$ is the largest.

On the right hand side, $\psi(x) \mathbf{1}_{v^{\prime-1},-c / 2}$ consists of all characters of level less than or equal to $c / 2$, except those differ from $v^{\prime-1}$ by a lower level characters. After the action of $\omega$, they will be supported on $v(x) \geq-c / 2$, as we already know. Multiplying with another $\psi$ won't change the support. By comparing the supports on both sides, we can get $n_{v^{\prime} v}=n_{v^{\prime}}$ for all $v$ for left hand side introduced by $\psi(-x)$ at $v(x)=c / 2+n_{v^{\prime}}$. On the right hand side, we get $n_{\mu}=-c$ for all $\mu$ of level $c / 2$ not differing from $v^{\prime}$ by lower level characters. But we know $n_{v^{\prime}}=n_{v^{\prime-1} w_{0}^{-1}}>-c$. To avoid contradiction, $v^{\prime-1} w_{0}^{-1}$ has to differ from $v^{\prime}$ by lower level characters. This implies $v^{\prime 2}$ itself is of lower level. But we will show this is impossible if $p \neq 2$ in the following lemma. Thus the proposition is proved.

Lemma A.2 Suppose that $p \neq 2$, and $v$ is a character of $O_{F}^{*}$ of level $n>1$. Then $v^{2}$ is still level $n$.

Proof Let $\varpi$ be a local uniformizer. $v$ being of level $n$ implies that there exist $b \in O_{F}^{*}$ such that $v\left(1+\varpi^{n-1} b\right) \neq 1$. If $p \neq 2$, then 2 is a unit. So

$$
v^{2}\left(1+\varpi^{n-1} b / 2\right)=v\left(1+\varpi^{n-1} b+\varpi^{2 n-2} b^{2} / 4\right)=v\left(1+\varpi^{n-1} b\right) \neq 1 .
$$

This means $v^{2}$ is still level $n$.

Remark A.3 When $p=2$ or/and $w_{\pi}$ is highly ramified, $n_{v}=\min \{-c,-2 i\}$ is still true for most cases, except when $c$ is even and $i=\frac{c}{2}$. Then we only expect $n_{v} \geq-c$. This result however is enough for some applications, see [12]. 
One can prove this result similarly as above, but one also need the observation that

$$
c\left(w_{\pi}\right) \leq c / 2
$$

when $w_{\pi}$ is highly ramified.

Received: 28 September 2016 Accepted: 3 October 2016

Published online: 25 November 2016

\section{References}

1. Böcherer, S., Schulze-Pillot, R.: On the central critical value of the triple product L-function in number theory (Paris, 1993-1994), London Math. Soc. Lecture Note Ser. 235, pp. 1-46, Cambridge Univ. Press, Cambridge (1996)

2. Bump, D.: Automorphic Forms and Representations. Cambridge Studies in Advanced Mathematics, vol. 55, Cambridge University Press, Cambridge (1997)

3. Bump, D.: The Rankin-Selberg method: an introduction and survey, automorphic representations, L-functions and applications: progress and prospects, pp. 41-73, Ohio State Univ. Math. Res. Inst. Publ., 11, de Gruyter, Berlin (2005)

4. Bruinier, J., Kudla, S.S., Yang, T.: Special values of Green functions at big CM points. Int. Math. Res. Not. IMRN 2012, no. 9, 1917-1967

5. Casselman, W.: On some results of Atkin and Lehner. Math. Ann. 201, 301-314 (1973)

6. Garrett, P.B.: Docomposition of Eisenstein series: Rankin triple products. Ann. Math. 2(125), 209-235 (1987)

7. Gross, B.H.: Local orders, root numbers, and modular curves. Am. J. Math. 110(6), 1153-1182 (1988)

8. Gross, B.H., Kudla, S.S.: Heights and the central critical values of triple product L-functions. Compos. Math. 81, 143-209 (1992)

9. Gross, B.H. Prasad, D.: Test vectors for linear forms. Math. Ann. 291(2), 343-355 (1991)

10. Harris, M., Kudla, S.S.: The central critical value of a triple product L-function. Ann. Math. 133, 605-672 (1991)

11. Harris, M., Kudla, S.S.: On a conjecture of Jacquet. Contributions to automorphic forms, geometry, and number theory, pp. 355-371, Johns Hopkins Univ. Press, Baltimore (2004)

12. Hu, Y.: The subconvexity bound for triple product L-function in level aspect, submitted. arXiv:1404.1636

13. $\mathrm{Hu}, \mathrm{Y}$.: Triple product formula and quantum unique ergodicity for modular curves of level $\mathrm{N}$, submitted. arXiv:1409.8173

14. Ichino, A.: Trilinear forms and the central values of triple product L-functions. Duke Math. J. 145(2), 281-307 (2008)

15. Jacquet, H., Langlands, R.P.: Automorphic forms on GL(2). Lecture Notes in Mathematics, vol. 114. Springer-Verlag, Berlin (1970)

16. Kable, A.C.: Asai L-functions and Jacquet's conjecture. Am. J. Math. 126(4), 789-820 (2004)

17. Kudla, S.S., Rallis, S.: A regularized Siegel-Weil formula: the first term identity. Ann. Math. 2(140), 1-80 (1994)

18. Piatetski-Shapiro, I., Rallis, S.: Rankin triple L functions. Compos. Math. 64, 31-115 (1987)

19. Popa, A.-A.: Central values of Rankin L-series over real quadratic fields. Compos. Math. 142(4), 811-866 (2006)

20. Prasad, D.: Trilinear forms for representations of GL(2) and local $\epsilon$-factors. Compos. Math. 75, 1-46 (1990)

21. Shimizu, H.: Theta series and automorphic forms on GL(2). Seminar on Modern Methods in Number Theory (Inst. Statist. Math., Tokyo, 1971), Paper No. 28, pp. 6, Inst. Statist. Math., Tokyo(1971)

22. Saito, H.: On Tunnell's formula for characters of GL(2). Compos. Math. 85(1), 99-108 (1993)

23. Tunnell, J.B.: Local epsilon-factors and characters of GL(2). Am. J. Math. 105(6), 1277-1307 (1983)

24. Waldspurger, J.L.: Sur les valeurs de certaines fonctions $L$ automorphes en leur centre de symétrie. Compos. Math. 54, $173-242(1985)$

25. Watson, T.C.: Rankin triple products and quantum chaos. Thesis (Ph. D.). Princeton University, pp. 81 (2002)

26. Xue, H.: Central values of Rankin L-functions. Int. Math. Res. Not. Art. ID 26150, pp. 41 (2006)

27. Yuan, X.Y., Zhang, S.W., Zhang, W.: The Gross-Zagier formula on Shimura curves. Ann. Math. Studies No. 184, Princeton University Press, Princeton and Oxford (2013)

28. Zhang, S.W.: Gross-Zagier formula for $G L_{2}$. Asian. J. Math. 5(2), 183-290 (2001)

\section{Submit your manuscript to a SpringerOpen ${ }^{\circ}$ journal and benefit from:}

- Convenient online submission

$\checkmark$ Rigorous peer review

- Immediate publication on acceptance

- Open access: articles freely available online

- High visibility within the field

- Retaining the copyright to your article

Submit your next manuscript at springeropen.com 